\title{
STANDARD SOLAR WAVE LENGTHS (3592-7148A)
}

\author{
By Keivin Burns, William F. Meggers, and C. C. Kiess
}

\begin{abstract}
In order to determine a series of solar wave lengths in the international system to serve as standards for a new table of solar wave lengths and to find the exact corrections which should be applied to Rowland's classical table of solar spectrum wave lengths, the Bureau of Standards and the Allegheny Observatory cooperated in measuring the wave lengths corresponding to selected dark (Fraunhofer) lines in the solar spectrum. The wave lengths were compared with those of standard neon lines by the Fabry and Perot étalon-interferometer method, both spectra being photographed simultaneously. More than 11,000 observations were made on 729 solar lines in the wave-length interval (octave) from $3592.027 \mathrm{~A}$ in the ultra-violet to $7148.159 \mathrm{~A}$ in the red. The majority of these standard solar wave lengths have individual probable errors smaller than 1 part in 4,500,000.
\end{abstract}

\section{CONTENTS}

III. Description of apparatus____._. 300

IV. Comparison sources_._._. 302

V. Measurement and reduction

VI. Results_........ 304

\section{INTRODUCTION}

The classical spectroscopic investigations of Professor Rowland culminating in his Preliminary Table of Solar Spectrum Wave Lengths, ${ }^{1}$ formed the foundation of the so-called Rowland scale of wave lengths which has been in more or less universal use for the past 35 years. Beginning with the best available value of the wave lengths corresponding to the sodium yellow lines, ${ }^{2}$ the relative wave lengths of a selected list of solar absorption lines ${ }^{3}$ were determined by the method of overlapping spectra from diffraction gratings.

Early in the present century it was recognized that Rowland's standards were too large by approximately 1 part in 30,000 , and relative errors of the order of 1 part in 100,000 were detected in different spectral regions. The International Union for Cooperation in Solar Research, ${ }^{4}$ later replaced by the International Astronomical

\footnotetext{
1 Rowland, Preliminary Table of Solar Spectrum Wave Lengths, The Univ. of Chicago Press; 1896.

${ }^{2}$ Rowland, Phil. Mag. (5), 23, p. 257; 1887 and 27, p. 479; 1889.

${ }^{3}$ Rowland, Phil. Mag. (5), 36, p. 49; 1893.

4 International Union for Cooperation in Solar Research, Trans. I, p. 230; 1906; II, pp. 109-174; 1908; III, p. 139; 1911; IV, p. 59; 1914.
} 
Union, ${ }^{5}$ has dealt with the situation since 1905 , and as a result of recommendations a new set of standard wave lengths has been determined and adopted to form the basis of the so-called international scale. This international system consists (1) of a primary standardviz, the wave length of cadmium red radiation which had previously been compared with the meter, and (2) several hundred secondary standards (3370-6750A) derived from the emission spectrum of the iron arc. These secondary standards were measured relative to the primary standard by means of the Fabry and Perot ${ }^{6}$ étalon-interferometer method. Certain neon lines which have been compared with cadmium by the same method have also been adopted as secondary standards.

This international system has gradually been displacing the older Rowland system, but there has been a tendency, especially in astrophysics, to retain the latter. The solar spectrum tables of Rowland are an almost inexhaustible mine of astrophysical study, but they could not be translated to the international scale without accurately determined corrections. On the other hand, the use of two different systems of standards caused considerable confusion in spectroscopy, and the only remedy for this double standard appeared to lie in a redetermination of solar. spectrum wave lengths on the international scale. In 1922 the International Astronomical Union expressed itself as follows: ${ }^{7}$

It is believed that the time has arrived for the determination of a series of solar wave lengths in the international system to serve as standards for a new table of solar wave lengths. It is recommended, therefore, that efforts be made to obtain at least three independent determinations of the wave lengths of a selected list of solar lines.

In 1923 the spectroscopy section of this bureau united with the Allegheny Observatory in a cooperative program of solar wavelength determinations, and results for one octave of the spectrum (3592-7148A) were completed in 1927 . These results were obtained in four overlapping spectral intervals. They were published in three installments according as the work in different spectral regions reached completion. ${ }^{8}$ In order that these results may be presented as a unified whole, they are republished here with the wave lengths (Table 1) in consecutive order and mean values replacing those reported twice in the slightly overlapping regions covered by the first publications. Similarly, the corrections to Rowland's wave

${ }^{5}$ International Astronomical Union, Trans. I, p. 35; 1922; II, p. 40; 1925.

${ }_{6}^{6}$ Fabry and Perot, Ann de Chim, et de Phys., 25, p. 98; 1902; Astrophys. J., 15, pp. 73 and 261; 1902.

${ }^{7}$ I. A. U. Transactions I, p. 36; 1922.

${ }^{8}$ Burns and Meggers, Standard solar wave lengths (4073-4754A), Pub. Allegheny Observatory, 6, No. 7; 1925. Burns and Kiess, Standard solar wave lengths (5805-7142A), Pub. Allegheny Observatory, 6, No. 8; 1927. Burns, Standard solar wave lengths (3592-4107A and 4761-5892A) Pub. Allegheny Observa. tory, 6, No. 9; 1927. 
lengths, previously reported in four sections, are now collected in one table (Table 2). A large number of additional "sun-arc" observations are included in Table 1, but the discussion of these is reserved for another paper. The main purpose of the one in hand is to present in complete and final form our observations on standard solar wave lengths. Only the essential facts relating to the method of observing, the description of the apparatus, and of the comparison sources will be given here. Further details may be found in the earlier publications.

\section{METHOD OF OBSERVING}

The method of observing is essentially that devised and first used by Fabry and Perot ${ }^{9}$ in 1902 for the measurement of 33 solar wave lengths (4643-6471A). It involves the use of a Fabry and Perot étalon interferometer combined with a prismatic or grating dispersing system, so that the orders of interference at various points in the spectrum may be compared. The method has become more or less familiar on account of its repeated use for the establishment of the international system of secondary standards in the emission spectrum of the iron arc. In the case of an absorption spectrum like that of the sun interference patterns similar to those produced by bright lines are obtained for dark (Fraunhofer) lines by a choice of order of interference and slit width such that a practical disappearance of the channeled spectrum from the continuous background is accomplished and interference fringes appear on the dark lines. The theory of interferences produced by the dark lines of the solar spectrum was discussed in some detail by Fabry and Buisson ${ }^{10}$ in 1910, but no further measurements of solar wave lengths were published until ours appeared. Similar observations have recently been made by this method at the Mount Wilson Observatory, and a portion of the results have been published. ${ }^{11}$

In order that solar spectrum standards may be placed on the same footing as the international secondary standards, they must be compared with the primary standard or its equivalent. Recognizing certain advantages of neon lines for such comparisons the International Astronomical Union ${ }^{12}$ considers the mean of eight or more well-determined neon wave lengths as practically equivalent to the primary standard. Our method of observing was arranged so that the solar spectrum and neon comparison spectrum could be recorded simultaneously. This procedure eliminates, or reduces to a minimum, the small uncontrollable disturbances which otherwise are likely to introduce systematic errors in precise comparisons of wave lengths.

\footnotetext{
- Fabry and Perot, see reference No. 6.

10 Fabry and Buisson, J. de Phys., 9, p. 197; 1910.

${ }_{11}$ Babcock, Astrophys. J., 65, p. 140, 1927.

12 I. A. U. Transactions II, p. 47; 1925.
} 


\section{DESCRIPTION OF APPARATUS}

All of the spectrograms were made at the Allegheny Observatory where the coelostat and spectrograph described by Schlesinger ${ }^{13}$ were employed, but the location and mounting of the latter instrument was changed. The former vertical mounting of the spectrograph proved to be too unstable for the rather long exposures required in this work. The instrument was, therefore, mounted horizontally on concrete piers in the basement of the observatory, where ideal stability and freedom from temperature changes were obtained. A coelostat mirror reflected the sunlight down the polar axis to another mirror which sent the light into the solar telescope and interferometer. The optical arrangement from this point onward is shown diagrammatically in Figure 1. After leaving the second coelostat mirror, the beam of sunlight passed through a color filter which was placed immediately in front of the solar lens. This filter reduced the heating effect of the sun in the interferometer, and at the same time removed the part of the solar spectrum corresponding to the neon comparison source, so that the spectrum of the latter could be simultaneously recorded in an auxiliary spectrograph camera. In the interval 5,800-6,500A the two spectra were photographed superposed, higher orders than the second being removed by a yellow-glass filter.

A telescope of $40 \mathrm{~cm}$ focal length forms an image of the sun in the interferometer, which is of the Fabry-Perot type. A diaphragm 6 $\mathrm{mm}$ in diameter near the first plate of the interferometer restricts the beam of the comparison spectrum to the size of the solar image. The sunlight from the telescope passes through a diagonal mirror, which serves to reflect the light of the laterally situated neon comparison lamp into the path of the solar beam. From this point on the solar and comparison beams follow identical paths until the grating separates them into spectra. For observing the photographic region of the solar spectrum this diagonal mirror is lightly silvered, reflecting rather more light than it transmits. For the longer solar wave lengths a thinner coat of silver is used, and for the region longer than $6600 \mathrm{~A}$ a clear-glass mirror has been used for some of the observations.

The interferometer is so mounted on a stand as to permit the necessary adjustments or to allow it to be lowered out of the path of the light so that the centering of the solar image and the comparison source on the grating may be more readily effected. The interferometer is turned $90^{\circ}$, in order to adjust the parallelism of the plates by means of a mercury lamp. The interferometer plates are thinly coated with silver, deposited cathodically. Some 10 to 15 images of a 40-watt lamp can be seen through a pair of plates. While a thicker

${ }^{13}$ Schlesinger, Pub. Allegheny Obs., 3, p. 99; 1914. 
coat would give slightly better interference, the increase in exposure time necessitated by the use of dense films more than offsets the slight gain in resolving power. The separators, or étalons, are made of invar.

A telescope of $25 \mathrm{~cm}$ focal length projects the ring system upon the slit of the spectrograph. The objective, of quartz and fluorite, is achromatic for the whole region of the solar spectrum observable at Allegheny Observatory. The interferometer is set at such a distance from this objective that the image of the diaphragm, $6 \mathrm{~mm}$ in diameter, is slightly smaller than the grating.

A gauge plate, or repère, having five fine parallel slots extending at right angles to the slit, is mounted on ways which allow it to slide almost in contact with the latter. Before the interferometer is placed in position this repère plate is slid in front of the slit. The intersections of the slit and the slots in this plate give five point-sources

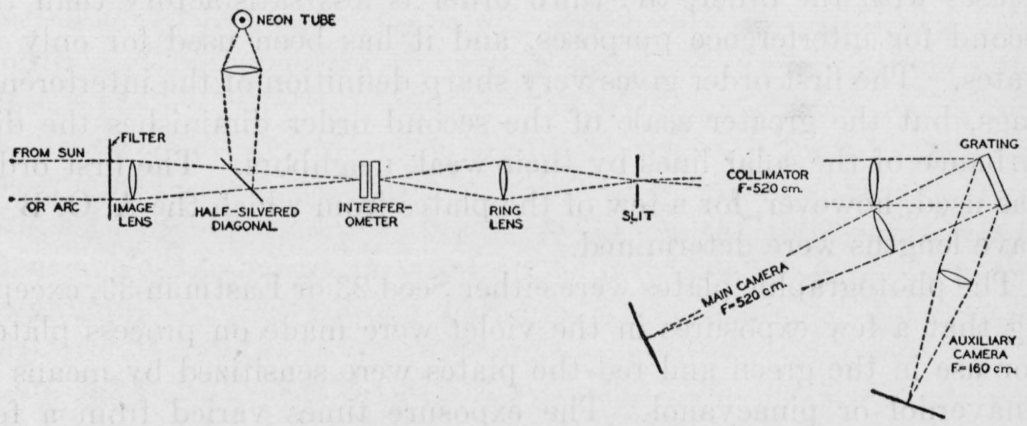

FIG. 1.-Diagram of interferometer and grating spectrograph for observing solar wave lengths

of light whose images are photographed in both the solar and comparison spectra. This furnishes a means of making sure that the same scale is used in computing the ring diameters in solar and comparison spectra, a matter of considerable importance when the two spectra differ greatly in wave length, but of little consequence when both spectra are of nearly the same wave length.

The collimator and camera lenses of the spectroscope are of $19 \mathrm{~cm}$ aperture and $520 \mathrm{~cm}$ focal length. Since parallel light falls upon the grating and the rulings are only $10 \mathrm{~cm}$ long, only the central $10 \mathrm{~cm}$ of each lens is useful in connection with the interferometer. The lenses are corrected for the photographic region, having minimum focal length at $4500 \mathrm{~A}$, at which point in the spectrum the plate is perpendicular to the axis of the camera. The grating, by Michelson, is ruled with 500 lines per millimeter, giving a linear scale of $3.65 \mathrm{~A}$ per $\mathrm{mm}$ in the first order.

To photograph the comparison spectrum, an auxiliary camera has been installed as shown in Figure 1. The lens of this camera is of $10 \mathrm{~cm}$ aperture and $160 \mathrm{~cm}$ focal length. The field is good over 
an angle of $5^{\circ}$, which is more than is required to photograph the neon spectrum in the region 5852 to $6598 \mathrm{~A}$. Since the solar camera is fixed with respect to the collimator, it is necessary to move the auxiliary camera as the various regions of the spectrum are brought into the solar camera. This comparison camera is arranged to rotate around an axis passing through the center of the grating; consequently the focus for the neon spectrum remains constant as the camera is rotated. For the region shorter than $6600 \mathrm{~A}$ the comparison spectrum is observed in the first order on the side of zero opposite from that on which the solar spectrum is photographed. For the longer region it is preferable to observe the comparison and solar spectra in the first and second orders, respectively, on the same side of zero.

The greater portion of the observing has been done with the grating adjusted to photograph the solar spectrum in the second order, giving a scale of $1.82 \mathrm{~A}$ per mm. Because of a slight astigmatism that increases with the order, the third order is less satisfactory than the second for interference purposes, and it has been used for only 10 plates. The first order gives very sharp definition of the interference rings, but the greater scale of the second order diminishes the disturbance of the solar lines by their weak neighbors. The first order was used, however, for a few of the plates from which the A. O. B. S. wave lengths were determined.

The photographic plates were either Seed 23 or Eastman 33, excepting that a few exposures in the violet were made on process plates. For use in the green and red the plates were sensitized by means of pinaverdol or pinacyanol. The exposure times varied from a few minutes to over two hours, depending on the condition of the sky, the state of the various silver coats, and the slit width.

Spectrograms were made in each portion of the solar spectrum with a variety of étalons and with two or more sets of interferometer plates. The various étalons were made of invar and had lengths of $3.75,5,6,7.5,8,10,12,15$, and $20 \mathrm{~mm}$.

\section{COMPARISON SOURCES}

The wave lengths of Table 1 were derived by simultaneous comparison with the red lines of neon, as already stated. The neon source was a lamp of the type described by Nutting. ${ }^{14}$ Its electrodes are aluminum disks $25 \mathrm{~mm}$ in diameter; the capillary is $10 \mathrm{~cm}$ long and has a bore of $2.5 \mathrm{~mm}$. An exciting current of about $50 \mathrm{~m}$. a. was delivered by 5,000 v. a. c. without capacity. The tube was observed exactly side on. The particular tube used in this investigation had been used previously in a comparison of neon wave lengths with the primary cadmium standard.

${ }_{14}$ Nutting, Bull. Bureau of Standards, 4, p. 511; 1908. 
The maximum pressure in the solar reversing layer where the Fraunhofer lines originate has been demonstrated to be so low that for spectroscopic purposes it may be regarded comparable with laboratory vacuum sources. For comparison of solar with terrestrial wave lengths we have employed the very convenient type of vacuum are designed and built by Curtis. ${ }^{15} \quad$ Using currents of $6 \mathrm{amp}$. or less, this arc operates without a cooling system and is in other respects so simple to manipulate that it would seem that this or a similar type of source should become a standard piece of laboratory equipment wherever wave lengths of a high order of accuracy are being determined. This arc was used as the source for all the sun-arc comparisons listed in Table 1. It was usually operated at pressures corresponding to 1 to $6 \mathrm{~cm}$ of mercury. All the vacuum arc wave lengths were derived from the neon standards by simultaneous comparison exactly in the same manner as the solar wave lengths.

\section{MEASUREMENT AND REDUCTION}

The diameters of two or, more often, three innermost fringes were measured for each of the wave lengths on a number of spectrograms ranging from 2 to 33 . In addition to the solar and neon spectra, each plate had on it the images of the slots in the repére plate at the slit, measurement of which furnished the scale factor used in the reductions.

The method of reduction employed by Buisson and Fabry ${ }^{16}$ was followed for about half of the plates, the remainder being reduced by the method described by Childs. ${ }^{17}$ In the former method the fractional order of interference at the center of the interference pattern is derived from measurements of the repère images and ring diameters, while in the latter it is deduced from ring diameters alone when three or more are available. Practically the same results are obtained from both methods, but the latter has the advantage of involving considerably less labor.

The order of interference was invariably obtained with the aid of the neon interferences, ${ }^{18}$ and the thickness of the interferometer, or double separation of the plates, was computed from each of six or more neon lines, the mean optical distance thus found being used to derive the solar wave lengths from their measured orders of interference. The values used for the neon lines were those compared with the primary cadmium standard; ${ }^{19}$ their mean value is regarded as practically equivalent to the primary standard itself.

Corrections for dispersion of phase upon reflection in the interferometer surfaces were made by the well-known device of comparing

\footnotetext{
15 Curtis, J. Opt. Soc. Am., 8, p. 697; 1924.

${ }^{16}$ Buisson and Fabry, J. de Phys., 7, p. 169; 1908, Astrophys. J., 28, p. 169; 1908.

${ }_{17}$ Childs, J. Sci. Inst., 3, pp. 97 and 129; 1926.

18 Meggers, Bull. Bureau of Standards, 12, p. 203; 1915.

10 Burns, Meggers, and Merrill, Bull. Bureau of Standards, 14, p. 765; 1918. I. A. U. Trans. I., p. 40, 1922. II, p. 44, 1925 .
} 
wave lengths first with low orders of interference and then with high values in which the differential phase change plays a proportionately smaller part. ${ }^{20}$ For this purpose wave lengths of the iron arc in vacuo were compared with neon standards by means of interferometers of $3.75,6,10$, and $20 \mathrm{~mm}$ separation.

Observations made under conditions of temperature and pressure of air which deviated from normal were corrected to their values at $15^{\circ} \mathrm{C}$. and $760 \mathrm{~mm} \mathrm{Hg}$ by the tables of Meggers and Peters. ${ }^{21}$

The proper corrections for motion of the earth relative to the sun were made for each spectrogram.

\section{RESULTS}

The first column of Table 1 contains the wave lengths of solar lines compared with red neon standards by means of simultaneous exposures as described above. The second column indicates the probable error of measurement. These probable errors are expressed in parts per million; thus " $\mathrm{A}$ " denotes a probable error of 1 part in $6,000,000$; "B," 1 part in $4,500,000$; "C," 1 part in $3,000,000$; and "D" probable errors greater than 1 part $3,000,000$. The number of independent comparisons of each line with the neon standards is indicated in column 3. The fourth, fifth, and sixth columns contain Rowland's wave length, chemical identification, and intensity for each line. An "s" attached to Rowland's wave length means that it was one of his standards. Column 7 shows the wave-length difference between Rowland and A. O. B. S. for the individual lines, and the next column gives the value of this difference interpolated from Table 2. In the ninth column are shown the differences between the wave lengths in the solar spectrum and the laboratory vacuum-arc values for the same lines, the unit being $0.001 \mathrm{~A}$. The final column gives the temperature class according to King and the pressure class to which the line belongs. The A. O. B. S. wavelength values are for air at $15^{\circ} \mathrm{C}$. and $760 \mathrm{~mm} \mathrm{Hg}$ pressure, while those of Rowland apply to air at $20^{\circ} \mathrm{C}$. and normal pressure. In both cases the source was integrated solar light.

In Table 2 are listed the corrections which must be applied to Rowland's wave lengths to reduce them to the international scale. These are taken from a smooth curve drawn through the plotted values of column 7 in Table 1 . The differences, Rowland minus A. O. B. S., run smoothly for the most part, but there are several marked discontinuities, notably at $4142 \mathrm{~A}$, at $5220 \mathrm{~A}$, and at $6870 \mathrm{~A}$. The smaller irregularity near $5800 \mathrm{~A}$ may not be real, as the observations between 5740 and $5850 \mathrm{~A}$ are of slightly inferior quality.

${ }^{20}$ Meggers, Bull. Bureau of Standards, 12, p. 199; 1915.

${ }_{21}$ Meggers and Peters, Bull. Bureau of Standards, 14, p. 724; 1918. 
A most encouraging feature of this comparison is an entire lack of an intensity equation. The values of the differences, Rowland minus A. O. B. S., are quite independent of the intensity of the solar line. After applying the interpolated corrections to Rowland's values, the mean of the residuals, Rowland minus A. O. B. S., is only $\pm 0.0015 \mathrm{~A}$.

TABLE 1.-A. O. B. S. solar wave lengths

[3592-7148A]

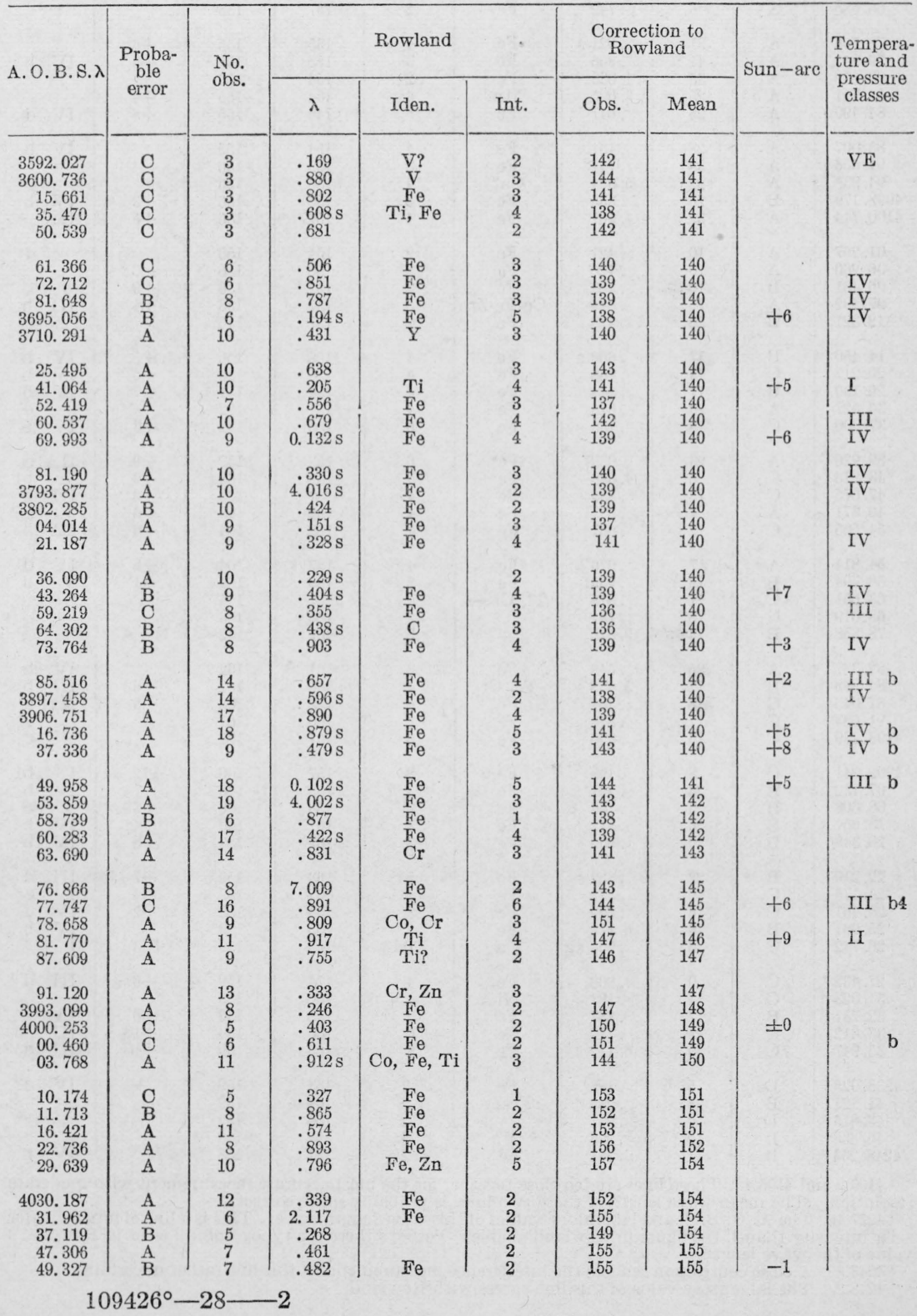


TABLE 1.-A.O. B.S. solar wave lengths-Continued

\begin{tabular}{|c|c|c|c|c|c|c|c|c|c|c|}
\hline \multirow{2}{*}{ A. O.B.S. $\lambda$} & \multirow{2}{*}{$\begin{array}{l}\text { Proba- } \\
\text { ble } \\
\text { error }\end{array}$} & \multirow{2}{*}{$\begin{array}{l}\text { No. } \\
\text { obs. }\end{array}$} & \multicolumn{3}{|c|}{ Rowland } & \multicolumn{2}{|c|}{$\begin{array}{l}\text { Correction to } \\
\text { Rowland }\end{array}$} & \multirow{2}{*}{ Sun-are } & \multirow{2}{*}{\multicolumn{2}{|c|}{$\begin{array}{l}\text { Temper } \\
\text { ture anc } \\
\text { pressure } \\
\text { classes }\end{array}$}} \\
\hline & & & $\lambda$ & Iden. & Int. & Obs. & Mean & & & \\
\hline $\begin{array}{r}4050.675 \\
53.824 \\
59.718 \\
62.446 \\
66.585\end{array}$ & $\begin{array}{l}\mathrm{A} \\
\mathrm{A} \\
\mathrm{A} \\
\mathrm{A} \\
\mathrm{A}\end{array}$ & $\begin{array}{r}8 \\
12 \\
8 \\
11 \\
6\end{array}$ & $\begin{array}{l}.830 \\
.981 \\
.872 \\
.599 \mathrm{~s} \\
.742\end{array}$ & $\begin{array}{c}\mathrm{Fe} \\
\mathrm{Fe}, \mathrm{Ti} \\
\mathrm{Fe} \\
\mathrm{Fe} \\
\mathrm{Fe}\end{array}$ & $\begin{array}{l}2 \\
3 \\
2 \\
5 \\
2\end{array}$ & $\begin{array}{l}155 \\
157 \\
154 \\
153 \\
157\end{array}$ & $\begin{array}{l}155 \\
155 \\
155 \\
155 \\
155\end{array}$ & +6 & III $b$ & b \\
\hline $\begin{array}{l}73.766 \\
79.841 \\
80.879 \\
82.941 \\
84.499\end{array}$ & $\begin{array}{l}\mathrm{A} \\
\mathrm{A} \\
\mathrm{A} \\
\mathrm{A} \\
\mathrm{A}\end{array}$ & $\begin{array}{l}31 \\
41 \\
35 \\
25 \\
25\end{array}$ & $\begin{array}{l}.921 \mathrm{~s} \\
.966 \\
1.033 \\
3.095 \\
.647\end{array}$ & $\begin{array}{l}\mathrm{Fe} \\
\mathrm{Fe} \\
\mathrm{Fe} \\
\mathrm{Mn} \\
\mathrm{Fe}\end{array}$ & $\begin{array}{l}4 \\
3 \\
2 \\
4 \\
5\end{array}$ & $\begin{array}{l}155 \\
155 \\
154 \\
154 \\
148\end{array}$ & $\begin{array}{l}155 \\
155 \\
155 \\
155 \\
155\end{array}$ & $\begin{array}{l}+6 \\
+3 \\
+4 \\
+8\end{array}$ & $\begin{array}{l}\text { IV } b \\
\text { IV } d\end{array}$ & b \\
\hline $\begin{array}{r}85.007 \\
91.556 \\
94.938 \\
4098.179 \\
4100.744\end{array}$ & $\begin{array}{l}\mathrm{A} \\
\mathrm{A} \\
\mathrm{A} \\
\mathrm{B} \\
\mathrm{A}\end{array}$ & $\begin{array}{l}28 \\
38 \\
22 \\
26 \\
32\end{array}$ & $\begin{array}{r}.161 \\
.711 \\
5.094 \\
.335 \\
.901\end{array}$ & $\begin{array}{l}\mathrm{Fe} \\
\mathrm{Fe} \\
\mathrm{Ca} ? \\
\mathrm{Fe} \\
\mathrm{Fe}\end{array}$ & $\begin{array}{l}4 \\
3 \\
4 \\
5 \\
4\end{array}$ & $\begin{array}{l}154 \\
155 \\
156 \\
156 \\
157\end{array}$ & $\begin{array}{l}155 \\
156 \\
156 \\
156 \\
156\end{array}$ & $\begin{array}{l}+3 \\
+5\end{array}$ & $\begin{array}{l}\text { IV } b \\
\text { IV } b\end{array}$ & b \\
\hline $\begin{array}{l}01.267 \\
06.2601 \\
06.431 \\
0.492 \\
12.321\end{array}$ & $\begin{array}{l}\mathrm{A} \\
\mathrm{A} \\
\mathrm{B} \\
\mathrm{A} \\
\mathrm{B}\end{array}$ & $\begin{array}{l}10 \\
10 \\
10 \\
25 \\
20\end{array}$ & $\begin{array}{l}.421 \\
.420 \\
.583 \\
.649 \\
.478\end{array}$ & $\begin{array}{c}\mathrm{Fe} \\
\mathrm{Fe} \\
\mathrm{Fe} \\
\mathrm{Ce}-\mathrm{Fe}-\mathrm{Zr} \\
\mathrm{Fe}\end{array}$ & $\begin{array}{l}2 \\
2 \\
2 \\
5 \\
2 \\
2\end{array}$ & $\begin{array}{l}154 \\
160 \\
152 \\
157 \\
157\end{array}$ & $\begin{array}{l}156 \\
156 \\
156 \\
156 \\
156\end{array}$ & $\begin{array}{l} \pm 0 \\
+9 \\
+4\end{array}$ & III $\mathrm{b}$ & b \\
\hline $\begin{array}{l}14.450 \\
20.212 \\
26.190 \\
36.527 \\
37.000\end{array}$ & $\begin{array}{l}\mathrm{B} \\
\mathrm{C} \\
\mathrm{B} \\
\mathrm{A} \\
\mathrm{C}\end{array}$ & $\begin{array}{l}17 \\
13 \\
17 \\
20 \\
18\end{array}$ & $\begin{array}{l}.606 \mathrm{~s} \\
.368 \\
.344 \\
.678 \\
.156\end{array}$ & $\begin{array}{l}\mathrm{Fe} \\
\mathrm{Fe} \\
\mathrm{Fe} \\
\mathrm{Fe} \\
\mathrm{Fe}\end{array}$ & $\begin{array}{l}4 \\
4 \\
4 \\
4 \\
6\end{array}$ & $\begin{array}{l}156 \\
156 \\
154 \\
151 \\
156\end{array}$ & $\begin{array}{l}156 \\
155 \\
154 \\
152 \\
152\end{array}$ & $\begin{array}{l}+5 \\
+6 \\
+7 \\
+3\end{array}$ & $\begin{array}{ll}\text { IV } & b \\
\text { IV } & b \\
\text { IV } & b \\
\text { IV } & b\end{array}$ & $\begin{array}{l}\mathrm{b} \\
\mathrm{b} \\
\mathrm{b} \\
\mathrm{d} \\
\mathrm{b}\end{array}$ \\
\hline $\begin{array}{l}39.936 \\
43.876 \\
47.675 \\
49.371 \\
54.505\end{array}$ & $\begin{array}{l}\mathrm{A} \\
\mathrm{C} \\
\mathrm{C} \\
\mathrm{A} \\
\mathrm{C}\end{array}$ & $\begin{array}{r}23 \\
6 \\
13 \\
7 \\
12\end{array}$ & $\begin{array}{l}0.089 \\
4.038 \\
.836 \\
.533 \\
.667\end{array}$ & $\begin{array}{l}\mathrm{Fe} \\
\mathrm{Fe} \\
\mathrm{Fe} \\
\mathrm{Fe} \\
\mathrm{Fe}\end{array}$ & $\begin{array}{r}6 \\
15 \\
4 \\
4 \\
4\end{array}$ & $\begin{array}{l}153 \\
162 \\
161 \\
162 \\
162\end{array}$ & $\begin{array}{l}152 \\
163 \\
163 \\
163 \\
164\end{array}$ & $\begin{array}{l}+9 \\
+9 \\
+6 \\
+6 \\
+6\end{array}$ & $\begin{array}{l}\text { IIA } \\
\text { III } \\
\text { III } \\
\text { III }\end{array}$ & $\begin{array}{l}\mathrm{a} \\
\mathrm{b} 1 \\
\mathrm{~b} \\
\mathrm{e} \\
\mathrm{b}\end{array}$ \\
\hline $\begin{array}{l}54.814 \\
58.794 \\
63.651 \\
68.619 \\
78.858\end{array}$ & $\begin{array}{l}\mathrm{A} \\
\mathrm{B} \\
\mathrm{C} \\
\mathrm{C} \\
\mathrm{B}\end{array}$ & $\begin{array}{r}17 \\
19 \\
11 \\
8 \\
13\end{array}$ & $\begin{array}{r}.976 \\
.959 \\
.818 \\
.784 \\
9.025\end{array}$ & $\begin{array}{c}\mathrm{Fe} \\
\mathrm{Fe} \\
\mathrm{Ti}, \mathrm{Cr}-\end{array}$ & $\begin{array}{l}4 \\
5 \\
4 \\
2 \\
3\end{array}$ & $\begin{array}{l}162 \\
165 \\
167 \\
165 \\
167\end{array}$ & $\begin{array}{l}164 \\
164 \\
164 \\
163 \\
162\end{array}$ & $\begin{array}{l}+8 \\
+1\end{array}$ & $\underset{V}{\text { IV }}$ & \\
\hline $\begin{array}{r}82.387 \\
84.898 \\
87.046 \\
91.683 \\
4198.639\end{array}$ & $\begin{array}{l}\mathrm{A} \\
\mathrm{B} \\
\mathrm{C} \\
\mathrm{C} \\
\mathrm{C}\end{array}$ & $\begin{array}{r}25 \\
12 \\
8 \\
11 \\
16\end{array}$ & $\begin{array}{l}.548 \\
5.058 \mathrm{~s} \\
.204 \\
.843 \\
.800\end{array}$ & $\begin{array}{c}\mathrm{Fe} \\
\mathrm{Fe}, \mathrm{Cr} \\
\mathrm{Fe} \\
\mathrm{Fe} \\
\mathrm{Fe}\end{array}$ & $\begin{array}{l}3 \\
4 \\
6 \\
3 \\
3 \\
3\end{array}$ & $\begin{array}{l}161 \\
160 \\
158 \\
160 \\
161\end{array}$ & $\begin{array}{l}162 \\
162 \\
162 \\
161 \\
161\end{array}$ & $\begin{array}{l}+6 \\
+8\end{array}$ & $\begin{array}{l}\text { IV } \\
\text { III } \\
\text { III } \\
\mathrm{b} \\
\mathrm{V}\end{array}$ & $\begin{array}{l}\mathrm{b} \\
\mathrm{b} \\
\mathrm{d} \\
\mathrm{e} \\
\mathrm{d}\end{array}$ \\
\hline $\begin{array}{r}4202.041 \\
07.132 \\
08.608 \\
13.651 \\
20.348\end{array}$ & $\begin{array}{l}\mathrm{C} \\
\mathrm{B} \\
\mathrm{B} \\
\mathrm{B} \\
\mathrm{B}\end{array}$ & $\begin{array}{r}8 \\
26 \\
20 \\
26 \\
30\end{array}$ & $\begin{array}{l}.198 \\
.291 \\
.766 \\
.812 \\
.509\end{array}$ & $\begin{array}{l}\mathrm{Fe} \\
\mathrm{Fe} \\
\mathrm{Fe} \\
\mathrm{Fe} \\
\mathrm{Fe}\end{array}$ & $\begin{array}{l}8 \\
3 \\
3 \\
3 \\
3 \\
3\end{array}$ & $\begin{array}{l}157 \\
159 \\
158 \\
161 \\
161\end{array}$ & $\begin{array}{l}160 \\
160 \\
160 \\
160 \\
159\end{array}$ & $\begin{array}{r}+12 \\
+3 \\
+4 \\
+8\end{array}$ & $\begin{array}{ll}\text { I } & b \\
\text { IV } & b \\
\text { V } & b \\
\text { IV } & b \\
\text { IV } & b\end{array}$ & $\begin{array}{l}\mathrm{b} 1 \\
\mathrm{~b} \\
\mathrm{~b} \\
\mathrm{~b} \\
\mathrm{~b}\end{array}$ \\
\hline $\begin{array}{l}22.219 \\
24.175 \\
25.461 \\
25.961 \\
26.742\end{array}$ & $\begin{array}{l}\mathrm{B} \\
\mathrm{C} \\
\mathrm{C} \\
\mathrm{B} \\
\mathrm{C}\end{array}$ & $\begin{array}{r}22 \\
9 \\
16 \\
16 \\
6\end{array}$ & $\begin{array}{c}.382 \mathrm{~s} \\
.337 \\
.619 \\
6.116 \\
.904 \mathrm{sg}\end{array}$ & $\begin{array}{l}\mathrm{Fe} \\
\mathrm{Fe} \\
\mathrm{Fe} \\
\mathrm{Fe} \\
\mathrm{Ca}\end{array}$ & $\begin{array}{c}5 \\
4 \\
3 \\
2 \\
20 \mathrm{~d} ?\end{array}$ & $\begin{array}{l}163 \\
162 \\
158 \\
155 \\
160\end{array}$ & $\begin{array}{l}159 \\
159 \\
159 \\
159 \\
159\end{array}$ & $\begin{array}{r}+7 \\
+4 \\
+7 \\
+13\end{array}$ & $\begin{array}{l}\text { III } \\
\text { IV } \\
\text { IV } \\
\text { IV } \\
\text { I }\end{array}$ & $\begin{array}{l}d \\
e \\
d \\
b\end{array}$ \\
\hline $\begin{array}{l}27.4322^{2} \\
31.023 \\
32.731 \\
33.612 \\
35.947\end{array}$ & $\begin{array}{l}\text { C } \\
\text { C } \\
\text { B } \\
\text { C } \\
\text { C }\end{array}$ & $\begin{array}{r}9 \\
11 \\
17 \\
13 \\
10\end{array}$ & $\begin{array}{r}.606 \\
.183 \\
.887 \\
.772 \\
6.112\end{array}$ & $\begin{array}{l}\mathrm{Fe} \\
\mathrm{Ni} \\
\mathrm{Fe} \\
\mathrm{Fe} \\
\mathrm{Fe}\end{array}$ & $\begin{array}{l}4 \\
4 \mathrm{~N} \\
2 \\
6 \\
8\end{array}$ & $\begin{array}{l}174 \\
160 \\
156 \\
160 \\
165\end{array}$ & $\begin{array}{l}159 \\
159 \\
159 \\
159 \\
159\end{array}$ & $\begin{array}{l}+6 \\
+6 \\
+10 \\
+10\end{array}$ & $\begin{array}{l}\text { III } \\
\text { IA } \\
\text { III } \\
\text { III }\end{array}$ & $\begin{array}{l}d \\
d 5 \\
d 5\end{array}$ \\
\hline $\begin{array}{c}4238.025 \\
41.121 \\
43.8153 \\
46.838 \\
428.83\end{array}$ & $\begin{array}{l}\mathrm{D} \\
\mathrm{B} \\
\mathrm{D} \\
\mathrm{B} \\
\mathrm{B}\end{array}$ & $\begin{array}{r}5 \\
25 \\
4 \\
10\end{array}$ & $\begin{array}{l}.188 \\
.285 \\
.981 \\
.996\end{array}$ & $\begin{array}{c}\mathrm{Fe} \\
\mathrm{Fe}-\mathrm{Zr} \\
\mathrm{Y} ?\end{array}$ & $\begin{array}{l}3 \\
2 \\
2 \\
5 \\
\end{array}$ & $\begin{array}{l}163 \\
164 \\
166 \\
158 \\
151\end{array}$ & $\begin{array}{l}159 \\
159 \\
159 \\
158 \\
158\end{array}$ & +3 & IV d & $\mathrm{d} ?$ \\
\hline
\end{tabular}

14106.2 and 4106.4. These lines are too close together for the most accurate measurement with moderate resolution. The mean wave length of these two lines is probably quite correct.

24227.4 . The A. O. B. S. and laboratory values of this wave length agree. This is a line of much greater solar intensity than 4, the figure in Rowland's table. Perhaps there is a typographical error in Rowland's value of the wave length.

3 4243.8. A close companion renders the interference measurements of this line rather uncertain.

4 4248.2. The laboratory value of this line agrees with Rowland. 
TABLe 1.-A. O. B. S. solar wave lengths-Continued

\begin{tabular}{|c|c|c|c|c|c|c|c|c|c|c|}
\hline \multirow{2}{*}{ A. O.B.S. $\lambda$} & \multirow{2}{*}{$\begin{array}{l}\text { Proba- } \\
\text { ble } \\
\text { error }\end{array}$} & \multirow{2}{*}{$\begin{array}{l}\text { No. } \\
\text { obs. }\end{array}$} & \multicolumn{3}{|c|}{ Rowland } & \multicolumn{2}{|c|}{$\begin{array}{l}\text { Correction to } \\
\text { Rowland }\end{array}$} & \multirow{2}{*}{ Sun-arc } & \multirow{2}{*}{\multicolumn{2}{|c|}{$\begin{array}{c}\text { Tempera } \\
\text { ture and } \\
\text { pressure } \\
\text { classes }\end{array}$}} \\
\hline & & & $\lambda$ & Iden. & Int. & Obs. & Mean & & & \\
\hline $\begin{array}{r}4250.129 \\
54.347 \\
57.660 \\
60.488 \\
65.264\end{array}$ & $\begin{array}{l}\mathrm{C} \\
\mathrm{C} \\
\mathrm{A} \\
\mathrm{C} \\
\mathrm{B}\end{array}$ & $\begin{array}{r}7 \\
5 \\
11 \\
7 \\
20\end{array}$ & $\begin{array}{l}.287 \\
.505 \mathrm{~s} \\
.815 \\
.640 \mathrm{~s} \\
.418\end{array}$ & $\begin{array}{l}\mathrm{Fe} \\
\mathrm{Cr} \\
\mathrm{Mn} \\
\mathrm{Fe} \\
\mathrm{Fe}\end{array}$ & $\begin{array}{r}8 \\
8 \\
2 \\
10 \\
2\end{array}$ & $\begin{array}{l}158 \\
158 \\
155 \\
152 \\
154\end{array}$ & $\begin{array}{l}158 \\
158 \\
158 \\
158 \\
158\end{array}$ & $\begin{array}{l}+10 \\
+10 \\
+14\end{array}$ & $\begin{array}{l}\text { III } \\
\text { II } \\
\text { II } \\
\text { III }\end{array}$ & $\begin{array}{l}c 5 \\
c 2\end{array}$ \\
\hline $\begin{array}{l}66.967 \\
68.754 \\
71.160 \\
71.774 \\
76.678\end{array}$ & $\begin{array}{l}\mathrm{C} \\
\mathrm{A} \\
\mathrm{C} \\
\mathrm{C} \\
\mathrm{B}\end{array}$ & $\begin{array}{r}11 \\
17 \\
7 \\
7 \\
13\end{array}$ & $\begin{array}{l}7.122 \\
.915 \\
.325 \\
.934 \mathrm{~s} \\
.836\end{array}$ & $\begin{array}{l}\mathrm{Fe} \\
\mathrm{Fe} \\
\mathrm{Fe} \\
\mathrm{Fe} \\
\mathrm{Zr}\end{array}$ & $\begin{array}{r}3 \\
2 \\
6 \\
15 \\
2\end{array}$ & $\begin{array}{l}155 \\
161 \\
165 \\
160 \\
158\end{array}$ & $\begin{array}{l}158 \\
158 \\
158 \\
158 \\
158\end{array}$ & $\begin{array}{l}+2 \\
+10 \\
+7 \\
+14\end{array}$ & $\begin{array}{l}\text { IV } \\
\text { IV } \\
\text { III } \\
\text { II }\end{array}$ & $\begin{array}{l}\mathrm{b} \\
\mathrm{b} \\
\mathrm{d} \\
\mathrm{b} 1\end{array}$ \\
\hline $\begin{array}{r}82.411 \\
90.384 \\
4291.471 \\
4318.658 \\
21.797\end{array}$ & $\begin{array}{l}\mathrm{B} \\
\mathrm{C} \\
\mathrm{B} \\
\mathrm{B} \\
\mathrm{B}\end{array}$ & $\begin{array}{r}19 \\
7 \\
26 \\
12 \\
9\end{array}$ & $\begin{array}{l}.565 \\
.542 \\
.630 \\
.817 \\
.961\end{array}$ & $\begin{array}{c}\mathrm{Fe} \\
\mathrm{Fe} \\
\mathrm{Fe} \\
\mathrm{Ca}, \mathrm{Mn} \text {. }\end{array}$ & $\begin{array}{l}5 \\
1 \\
2 \\
4 \\
2\end{array}$ & $\begin{array}{l}154 \\
158 \\
159 \\
159 \\
164\end{array}$ & $\begin{array}{l}158 \\
158 \\
158 \\
160 \\
161\end{array}$ & $\begin{array}{l}+8 \\
+7\end{array}$ & $\begin{array}{l}\text { III } \\
\text { IA }\end{array}$ & $\begin{array}{l}\mathrm{b} 1 \\
\mathrm{a} 3\end{array}$ \\
\hline $\begin{array}{l}25.773 \\
26.758 \\
27.110 \\
31.649 \\
37.055\end{array}$ & $\begin{array}{l}\mathrm{D} \\
\mathrm{B} \\
\mathrm{A} \\
\mathrm{B} \\
\mathrm{C}\end{array}$ & $\begin{array}{r}5 \\
14 \\
18 \\
18 \\
20\end{array}$ & $\begin{array}{l}.939 \mathrm{~s} \\
.923 \\
.274 \\
.811 \\
.216\end{array}$ & $\begin{array}{l}\mathrm{Fe} \\
\mathrm{Fe} \\
\mathrm{Fe} \\
\mathrm{Ni} \\
\mathrm{Fe}\end{array}$ & $\begin{array}{l}8 \\
2 \\
3 \\
2 \\
5\end{array}$ & $\begin{array}{l}166 \\
165 \\
164 \\
162 \\
161\end{array}$ & $\begin{array}{l}161 \\
161 \\
161 \\
162 \\
162\end{array}$ & +12 & $\begin{array}{l}\text { II } \\
\text { V } \\
\text { II } \\
\text { II }\end{array}$ & $\begin{array}{l}\mathrm{b} 1 \\
\mathrm{~b} \\
\mathrm{~b} 3\end{array}$ \\
\hline $\begin{array}{l}37.927 \\
38.265 \\
43.703 \\
46.557 \\
47.238\end{array}$ & $\begin{array}{l}\text { A } \\
\text { C } \\
\text { B } \\
\text { C } \\
\text { B }\end{array}$ & $\begin{array}{l}17 \\
17 \\
18 \\
13 \\
17\end{array}$ & $\begin{array}{r}8.084 \\
.430 \\
.861 \\
.725 \\
.403\end{array}$ & $\begin{array}{l}\mathrm{Ti} \\
\mathrm{Fe} \\
\mathrm{Fe} \\
\mathrm{Fe} \\
\mathrm{Fe}\end{array}$ & $\begin{array}{l}4 \\
1 \\
2 \\
2 \\
1\end{array}$ & $\begin{array}{l}157 \\
165 \\
158 \\
168 \\
165\end{array}$ & $\begin{array}{l}162 \\
162 \\
162 \\
162 \\
162\end{array}$ & $\begin{array}{l}+11 \\
+2 \\
+4 \\
+5\end{array}$ & VE & \\
\hline $\begin{array}{l}48.946 \\
51.548 \\
58.504 \\
59.621 \\
60.799\end{array}$ & $\begin{array}{l}\mathrm{B} \\
\mathrm{B} \\
\mathrm{B} \\
\mathrm{D} \\
\mathrm{A}\end{array}$ & $\begin{array}{r}16 \\
12 \\
13 \\
6 \\
11\end{array}$ & $\begin{array}{l}9.107 \\
.711 \\
.670 \\
.748 \mathrm{~s} \\
.958\end{array}$ & $\begin{array}{c}\mathrm{Fe} \\
\mathrm{Fe} \\
\mathrm{Fe} \\
\mathrm{Cr} \\
\mathrm{Fe}, \mathrm{Zr}\end{array}$ & $\begin{array}{l}2 \\
2 \\
2 \\
3 \\
1\end{array}$ & $\begin{array}{l}161 \\
163 \\
166 \\
163 \\
159\end{array}$ & $\begin{array}{l}162 \\
162 \\
161 \\
161 \\
161\end{array}$ & $\begin{array}{r}+10 \\
+6 \\
+4\end{array}$ & $\begin{array}{l}\text { IV } \\
\text { IV } \\
\text { I }\end{array}$ & $\begin{array}{l}\mathrm{b} \\
\mathrm{b}\end{array}$ \\
\hline $\begin{array}{l}65.903 \\
67.910 \\
69.779 \\
73.564 \\
75.947\end{array}$ & $\begin{array}{l}\mathrm{B} \\
\mathrm{A} \\
\mathrm{C} \\
\mathrm{B} \\
\mathrm{C}\end{array}$ & $\begin{array}{l}28 \\
24 \\
16 \\
18 \\
25\end{array}$ & $\begin{array}{l}6.061 \\
8.071 \\
.94 \mathbf{b s} \\
.727 \\
6.107 \mathrm{~s}\end{array}$ & $\begin{array}{l}\mathrm{Fe} \\
\mathrm{Fe} \\
\mathrm{Fe} \\
\mathrm{Fe} \\
\mathrm{Fe}\end{array}$ & $\begin{array}{l}2 \\
2 \\
4 \\
2 \\
6\end{array}$ & $\begin{array}{l}158 \\
161 \\
162 \\
163 \\
160\end{array}$ & $\begin{array}{l}161 \\
161 \\
161 \\
161 \\
160\end{array}$ & $\begin{array}{l}+4 \\
+7 \\
+1 \\
+17\end{array}$ & $\begin{array}{l}\text { IIIA } \\
\text { III } \\
\text { I }\end{array}$ & $\begin{array}{l}\mathrm{A} \text { a } \\
\mathrm{b} 3 \\
\mathrm{a} 3\end{array}$ \\
\hline $\begin{array}{l}77.793 \\
79.241 \\
83.563 \\
87.897 \\
88.414\end{array}$ & $\begin{array}{l}\text { B } \\
\text { D } \\
\text { C } \\
\text { C } \\
\text { C }\end{array}$ & $\begin{array}{r}18 \\
5 \\
9 \\
22 \\
7\end{array}$ & $\begin{array}{l}.948 \\
.396 \\
.720 \mathrm{~s} \\
8.057 \\
.571\end{array}$ & $\begin{array}{c}\mathrm{Fe} \\
\mathrm{V} \\
\mathrm{Fe} \\
\mathrm{Fe}, \mathrm{Co} \\
\mathrm{Fe}\end{array}$ & $\begin{array}{r}1 \\
4 \\
15 \\
2 \\
3\end{array}$ & $\begin{array}{l}155 \\
155 \\
157 \\
160 \\
157\end{array}$ & $\begin{array}{l}160 \\
159 \\
159 \\
159 \\
159\end{array}$ & $\begin{array}{r}+19 \\
+7\end{array}$ & $\begin{array}{l}\text { II } \\
\text { IV } \\
\text { IV }\end{array}$ & $\begin{array}{l}\mathrm{b} 1 \\
\mathrm{~b} \\
\mathrm{~d} ?\end{array}$ \\
\hline $\begin{array}{r}89.252 \\
94.066 \\
98.020 \\
4399.775 \\
4404.767\end{array}$ & $\begin{array}{l}\text { A } \\
\text { A } \\
\text { B } \\
\text { A } \\
\text { C }\end{array}$ & $\begin{array}{r}18 \\
10 \\
16 \\
15 \\
9\end{array}$ & $\begin{array}{l}.413 \\
.225 \\
.178 \\
.935 \\
.927 \mathrm{~s}\end{array}$ & $\begin{array}{c}\mathrm{Fe}- \\
\mathrm{Ti} ? \\
\underset{\mathrm{Ti}}{\mathrm{Fe}} \mathrm{Cr}\end{array}$ & $\begin{array}{r}2 \\
2 \\
1 \\
3 \\
10\end{array}$ & $\begin{array}{l}161 \\
159 \\
158 \\
160 \\
160\end{array}$ & $\begin{array}{l}159 \\
159 \\
150 \\
160 \\
160\end{array}$ & +17 & IIA & $a$ \\
\hline $\begin{array}{c}08.204 \\
15.1445 \\
16.829 \\
22.572 \\
4425.445\end{array}$ & $\begin{array}{l}\mathrm{B} \\
\mathrm{B} \\
\mathrm{B} \\
\mathrm{B} \\
\mathrm{B}\end{array}$ & $\begin{array}{l}14 \\
13 \\
17 \\
16 \\
19\end{array}$ & $\begin{array}{l}.364 \\
.263 \mathrm{~s} \\
.985 \\
.741 \\
.608 \mathrm{~s}\end{array}$ & $\begin{array}{c}\mathrm{V} \\
\mathrm{Fe} \\
\mathrm{Fa}, \mathrm{Y}\end{array}$ & $\begin{array}{l}2 \\
8 \\
2 \\
3 \\
4\end{array}$ & $\begin{array}{l}160 \\
149 \\
156 \\
169 \\
163\end{array}$ & $\begin{array}{l}160 \\
161 \\
161 \\
161 \\
161\end{array}$ & $\begin{array}{l}+22 \\
+3\end{array}$ & $\begin{array}{l}\text { II } \\
\text { III } \\
\text { I }\end{array}$ & $\begin{array}{l}\mathrm{b} 1 \\
\mathrm{~b} 3\end{array}$ \\
\hline $\begin{array}{l}27.323 \\
30.195 \\
30.621 \\
32.572 \\
33.230\end{array}$ & $\begin{array}{l}\mathrm{B} \\
\mathrm{D} \\
\mathrm{B} \\
\mathrm{B} \\
\mathrm{C}\end{array}$ & $\begin{array}{r}16 \\
6 \\
29 \\
16 \\
16\end{array}$ & $\begin{array}{l}.482 \\
.356 \\
.785 \\
.736 \\
.390\end{array}$ & $\begin{array}{l}\mathrm{Fe} \\
\mathrm{Fe} \\
\mathrm{Fe} \\
\mathrm{Fe} \\
\mathrm{Fe}\end{array}$ & $\begin{array}{l}5 \\
1 \\
3 \\
1 \\
3\end{array}$ & $\begin{array}{l}159 \\
161 \\
164 \\
164 \\
160\end{array}$ & $\begin{array}{l}162 \\
162 \\
162 \\
162 \\
162\end{array}$ & $\begin{array}{r}+13 \\
+7 \\
+10\end{array}$ & $\begin{array}{l}\text { I } \\
\text { IV } \\
\text { III } \\
\text { IV }\end{array}$ & $\begin{array}{r}\mathrm{a} 3 \\
\mathrm{c} 4 \\
\mathrm{e}\end{array}$ \\
\hline $\begin{array}{r}33.783 \\
35.157 \\
35.688 \\
39.888 \\
4442.353\end{array}$ & $\begin{array}{l}\text { C } \\
\text { D } \\
B \\
A \\
B\end{array}$ & $\begin{array}{r}7 \\
5 \\
15 \\
24 \\
20\end{array}$ & $\begin{array}{l}.948 \\
.321 \\
.851 \mathrm{~s} \\
0.054 \\
.510\end{array}$ & $\begin{array}{l}\mathrm{Fe} \\
\mathrm{Fe} \\
\mathrm{Ca} \\
\mathrm{Fe} \\
\mathrm{Fe}\end{array}$ & $\begin{array}{l}1 \\
2 \\
4 \\
1 \\
6\end{array}$ & $\begin{array}{l}165 \\
164 \\
163 \\
166 \\
157\end{array}$ & $\begin{array}{l}162 \\
162 \\
162 \\
163 \\
163\end{array}$ & $\begin{array}{l}+1 \\
+9\end{array}$ & $\begin{array}{l}\text { IIA } \\
\text { I } \\
\text { IV } \\
\text { IV }\end{array}$ & $\begin{array}{l}\mathrm{d} \\
\mathrm{a} \\
\mathrm{c} 4\end{array}$ \\
\hline
\end{tabular}

3 4415.1. The wave length of this line is no doubt affected by close companions. The laboratory value agrees with Rowland. 
TABLE 1.-A. O. B. S. solar wave lengths-Continued

\begin{tabular}{|c|c|c|c|c|c|c|c|c|c|c|}
\hline \multirow{2}{*}{ A. O.B.S. $\lambda$} & \multirow{2}{*}{$\begin{array}{l}\text { Proba- } \\
\text { ble } \\
\text { error }\end{array}$} & \multirow{2}{*}{$\begin{array}{l}\text { No. } \\
\text { obs. }\end{array}$} & \multicolumn{3}{|c|}{ Rowland } & \multicolumn{2}{|c|}{$\begin{array}{l}\text { Correction to } \\
\text { Rowland }\end{array}$} & \multirow{2}{*}{ Sun-are } & \multirow{2}{*}{\multicolumn{2}{|c|}{$\begin{array}{l}\text { Tempera- } \\
\text { ture and } \\
\text { pressure } \\
\text { classes }\end{array}$}} \\
\hline & & & $\lambda$ & Iden & Int. & Obs. & Mean & & & \\
\hline $\begin{array}{c}4443.202 \\
43.812 \\
45.476 \\
47.737{ }^{6} \\
51.588\end{array}$ & $\begin{array}{l}\mathrm{B} \\
\mathrm{B} \\
\mathrm{B} \\
\mathrm{A} \\
\mathrm{B}\end{array}$ & $\begin{array}{l}13 \\
15 \\
13 \\
16 \\
16\end{array}$ & $\begin{array}{l}.365 \\
.976 \\
.641 \\
.892 \mathrm{~s} \\
.752\end{array}$ & $\begin{array}{l}\mathrm{Fe} \\
\mathrm{Ti} \\
\mathrm{Fe} \\
\mathrm{Fe} \\
\mathrm{Mn}\end{array}$ & $\begin{array}{l}3 \\
5 \\
1 \\
6 \\
3\end{array}$ & $\begin{array}{l}163 \\
164 \\
165 \\
155 \\
164\end{array}$ & $\begin{array}{l}163 \\
163 \\
163 \\
163 \\
163\end{array}$ & $\begin{array}{r}+8 \\
+10 \\
+6 \\
+20\end{array}$ & $\begin{array}{l}\text { III } \\
\text { VE } \\
\text { IA } \\
\text { III } \\
\text { II }\end{array}$ & $\begin{array}{l}\text { b3 } \\
\mathrm{c} 4\end{array}$ \\
\hline $\begin{array}{l}54.388 \\
56.332 \\
59.756 \\
61.661 \\
66.566\end{array}$ & $\begin{array}{l}\mathrm{A} \\
\mathrm{B} \\
\mathrm{A} \\
\mathrm{B} \\
\mathrm{B}\end{array}$ & $\begin{array}{l}30 \\
10 \\
15 \\
24 \\
22\end{array}$ & $\begin{array}{l}.552 \\
.497 \\
.922 \\
.818 \\
.727\end{array}$ & $\begin{array}{l}\mathrm{Fe} \\
\mathrm{Fe} \\
\mathrm{V} \\
\mathrm{Fe} \\
\mathrm{Fe}\end{array}$ & $\begin{array}{l}3 \\
1 \\
1 \\
4 \\
5\end{array}$ & $\begin{array}{l}164 \\
165 \\
166 \\
157 \\
161\end{array}$ & $\begin{array}{l}163 \\
163 \\
163 \\
163 \\
163\end{array}$ & $\begin{array}{r}+7 \\
\\
+8 \\
+14\end{array}$ & $\begin{array}{l}\text { III } \\
\text { II }\end{array}$ & $\begin{array}{l}\mathrm{b} 3 \\
\\
\mathrm{a} 3 \\
\mathrm{~b} 4\end{array}$ \\
\hline $\begin{array}{l}70.136 \\
70.486 \\
81.617 \\
84.229 \\
85.682\end{array}$ & $\begin{array}{l}\mathrm{D} \\
\mathrm{A} \\
\mathrm{A} \\
\mathrm{A} \\
\mathrm{A}\end{array}$ & $\begin{array}{r}6 \\
12 \\
13 \\
17 \\
17\end{array}$ & $\begin{array}{l}.300 \\
.648 \\
.782 \\
.392 \\
.846\end{array}$ & $\begin{array}{c}\mathrm{Mn} \\
\mathrm{Ni}-\mathrm{Zr} \\
\mathrm{Fe} \\
\mathrm{Fe} \\
\mathrm{Fe}\end{array}$ & $\begin{array}{l}1 \\
2 \\
1 \\
4 \\
3\end{array}$ & $\begin{array}{l}164 \\
162 \\
165 \\
163 \\
164\end{array}$ & $\begin{array}{l}163 \\
163 \\
163 \\
163 \\
163\end{array}$ & $\begin{array}{l}+9 \\
+6\end{array}$ & $\begin{array}{l}\text { III } \\
\text { IV } \\
\text { IV }\end{array}$ & d \\
\hline $\begin{array}{r}91.410 \\
94.575 \\
4496.864 \\
4501.280 \\
02.220\end{array}$ & $\begin{array}{l}\mathrm{B} \\
\mathrm{A} \\
\mathrm{B} \\
\mathrm{A} \\
\mathrm{A}\end{array}$ & $\begin{array}{l}16 \\
14 \\
17 \\
16 \\
15\end{array}$ & $\begin{array}{l}.570 \\
.738 \mathrm{~s} \\
7.023 \mathrm{~s} \\
.448 \mathrm{~s} \\
.388\end{array}$ & $\begin{array}{l}\mathrm{Fe} \\
\mathrm{Cr} \\
\mathrm{Ti}- \\
\mathrm{Mn}\end{array}$ & $\begin{array}{l}2 \\
6 \\
3 \\
5 \\
2\end{array}$ & $\begin{array}{l}160 \\
163 \\
159 \\
168 \\
168\end{array}$ & $\begin{array}{l}164 \\
164 \\
164 \\
165 \\
165\end{array}$ & +12 & $\begin{array}{l}\text { III } \\
\text { III }\end{array}$ & $\mathrm{ct}$ \\
\hline $\begin{array}{l}04.834 \\
08.290 \\
12.741 \\
17.536 \\
25.149\end{array}$ & $\begin{array}{l}\mathrm{B} \\
\mathrm{A} \\
\mathrm{A} \\
\mathrm{A} \\
\mathrm{A}\end{array}$ & $\begin{array}{l}16 \\
23 \\
22 \\
17 \\
16\end{array}$ & $\begin{array}{l}5.003 \\
.455 \mathrm{~s} \\
.906 \\
.702 \\
.314\end{array}$ & $\begin{array}{l}\mathrm{Fe} \\
\mathrm{Fe} ?- \\
\mathrm{Ti} \\
\mathrm{Fe} \\
\mathrm{Fe}\end{array}$ & $\begin{array}{l}1 \\
4 \\
3 \\
3 \\
5\end{array}$ & $\begin{array}{l}169 \\
165 \\
165 \\
166 \\
165\end{array}$ & $\begin{array}{l}165 \\
165 \\
165 \\
166 \\
167\end{array}$ & $\begin{array}{r}+6 \\
+7 \\
+12 \\
+11\end{array}$ & $\begin{array}{l}\text { II } \\
\text { IV }\end{array}$ & d? \\
\hline $\begin{array}{l}26.934 \\
28.631 \\
31.631 \\
34.785 \\
41.524\end{array}$ & $\begin{array}{l}\text { B } \\
\text { B } \\
\text { A } \\
\text { A } \\
\text { A }\end{array}$ & $\begin{array}{l}15 \\
13 \\
21 \\
17 \\
22\end{array}$ & $\begin{array}{l}7.101 \\
.798 \\
.801 \\
.953 \\
.690\end{array}$ & $\begin{array}{l}\mathrm{Ca} ? \\
\mathrm{Fe} \\
\mathrm{Fe} \\
\mathrm{Ti} \\
\mathrm{Cr}\end{array}$ & $\begin{array}{l}3 \\
8 \\
2 \\
4 \\
2\end{array}$ & $\begin{array}{l}167 \\
167 \\
170 \\
168 \\
166\end{array}$ & $\begin{array}{l}167 \\
167 \\
167 \\
167 \\
168\end{array}$ & +17 & II & $\begin{array}{l}\mathrm{c} 4 \\
\mathrm{~b} 3\end{array}$ \\
\hline $\begin{array}{l}47.855 \\
48.772 \\
50.775 \\
54.042 \\
58.653\end{array}$ & $\begin{array}{l}\mathrm{A} \\
\mathrm{A} \\
\mathrm{A} \\
\mathrm{B} \\
\mathrm{A}\end{array}$ & $\begin{array}{r}17 \\
16 \\
15 \\
8 \\
7\end{array}$ & $\begin{array}{l}8.024 \\
.938 \\
.942 \\
.211 \mathrm{~s} \\
.827\end{array}$ & $\begin{array}{l}\mathrm{Fe} \\
\mathrm{Ti} \\
\mathrm{Fe} ? \\
\mathrm{Ba} \\
\mathrm{Cr} ?\end{array}$ & $\begin{array}{l}3 \\
2 \\
2 \\
8 \\
3\end{array}$ & $\begin{array}{r}169 \\
166 \\
167 \\
-169 \\
174\end{array}$ & $\begin{array}{l}168 \\
168 \\
168 \\
169 \\
169\end{array}$ & $\begin{array}{l}+8 \\
+8 \\
+5\end{array}$ & $\begin{array}{l}\text { II } \\
\text { II }\end{array}$ & $\begin{array}{l}\mathrm{b} \\
\mathrm{d}\end{array}$ \\
\hline $\begin{array}{l}60.094 \\
63.769 \\
71.103 \\
71.983 \\
74.727\end{array}$ & $\begin{array}{l}\mathrm{B} \\
\mathrm{B} \\
\mathrm{A} \\
\mathrm{A} \\
\mathrm{B}\end{array}$ & $\begin{array}{l}10 \\
20 \\
20 \\
15 \\
10\end{array}$ & $\begin{array}{l}.266 \\
.939 \mathrm{~s} \\
.275 \mathrm{~s} \\
.156 \mathrm{~s} \\
.899\end{array}$ & $\begin{array}{l}\mathrm{Fe} \\
\mathrm{Ti} \\
\mathrm{Mg} \\
\mathrm{Ti}- \\
\mathrm{Fe}\end{array}$ & $\begin{array}{l}2 \\
4 \\
5 \\
6 \\
2\end{array}$ & $\begin{array}{l}172 \\
170 \\
172 \\
173 \\
172\end{array}$ & $\begin{array}{l}170 \\
171 \\
172 \\
172 \\
172\end{array}$ & $\begin{array}{l}+7 \\
+8\end{array}$ & $\begin{array}{l}\text { VE } \\
\text { IA }\end{array}$ & d \\
\hline $\begin{array}{r}76.340 \\
78.560 \\
4587.135 \\
88.207 \\
89.955\end{array}$ & $\begin{array}{l}\mathrm{A} \\
\mathrm{A} \\
\mathrm{B} \\
\mathrm{A} \\
\mathrm{A}\end{array}$ & $\begin{array}{l}15 \\
17 \\
20 \\
16 \\
15\end{array}$ & $\begin{array}{l}.512 \\
.732 \mathrm{~s} \\
.308 \\
.381 \mathrm{~s} \\
0.126 \mathrm{~s}\end{array}$ & $\begin{array}{l}\mathrm{Ca} \\
\mathrm{Fe}\end{array}$ & $\begin{array}{l}2 \\
3 \\
2 \\
3 \\
3\end{array}$ & $\begin{array}{l}172 \\
172 \\
173 \\
174 \\
171\end{array}$ & $\begin{array}{l}172 \\
172 \\
172 \\
173 \\
173\end{array}$ & +7 & II & \\
\hline $\begin{array}{r}95.367 \\
4598.127 \\
4602.008 \\
02.951 \\
07.657\end{array}$ & $\begin{array}{l}\mathrm{A} \\
\mathrm{A} \\
\mathrm{A} \\
\mathrm{A} \\
\mathrm{A}\end{array}$ & $\begin{array}{l}21 \\
14 \\
17 \\
17 \\
23\end{array}$ & $\begin{array}{c}.540 \\
.303 \\
.183 \mathrm{~s} \\
3.126 \\
.831\end{array}$ & $\begin{array}{l}\mathrm{Fe} \\
\mathrm{Fe} \\
\mathrm{Fe} \\
\mathrm{Fe} \\
\mathrm{Fe}\end{array}$ & $\begin{array}{l}2 \\
3 \\
3 \\
6 \\
4\end{array}$ & $\begin{array}{l}173 \\
176 \\
175 \\
175 \\
174\end{array}$ & $\begin{array}{l}173 \\
174 \\
174 \\
174 \\
174\end{array}$ & $\begin{array}{l}+9 \\
+10 \\
+7 \\
+10 \\
+11\end{array}$ & $\underset{V}{\text { IB }}$ & $d$ \\
\hline $\begin{array}{l}16.133 \\
17.277 \\
20.520 \\
25.055 \\
30.128\end{array}$ & $\begin{array}{l}\mathrm{A} \\
\mathrm{A} \\
\mathrm{A} \\
\mathrm{A} \\
\mathrm{A}\end{array}$ & $\begin{array}{l}17 \\
16 \\
21 \\
19 \\
17\end{array}$ & $\begin{array}{l}.305 \\
.452 \\
.693 \\
.227 \\
.306\end{array}$ & $\begin{array}{l}\mathrm{Cr} \\
\mathrm{Ti} \\
\mathrm{Fe} \\
\mathrm{Fe} \\
\mathrm{Fe}\end{array}$ & $\begin{array}{l}4 \\
3 \\
1 \\
5 \\
4\end{array}$ & $\begin{array}{l}172 \\
175 \\
173 \\
172 \\
178\end{array}$ & $\begin{array}{l}174 \\
174 \\
174 \\
174 \\
174\end{array}$ & $\begin{array}{l}+8 \\
+10 \\
+8\end{array}$ & II & d \\
\hline $\begin{array}{l}35.855 \\
37.511 \\
38.019 \\
43.472 \\
47.443\end{array}$ & $\begin{array}{l}\mathrm{A} \\
\mathrm{C} \\
\mathrm{A} \\
\mathrm{A} \\
\mathrm{C}\end{array}$ & $\begin{array}{r}15 \\
7 \\
16 \\
23 \\
7\end{array}$ & $\begin{array}{l}6.027 \\
.685 \mathrm{~s} \\
.193 \mathrm{~s} \\
.645 \mathrm{~s} \\
.617\end{array}$ & $\begin{array}{l}\mathrm{Fe} \\
\mathrm{Fe} \\
\mathrm{Fe} \\
\mathrm{Fe} \\
\mathrm{Fe}\end{array}$ & $\begin{array}{l}2 \\
5 \\
4 \\
4 \\
4\end{array}$ & $\begin{array}{l}172 \\
174 \\
174 \\
173 \\
174\end{array}$ & $\begin{array}{l}173 \\
173 \\
173 \\
173 \\
173\end{array}$ & $\begin{array}{l}+8 \\
+6 \\
+11 \\
+8 \\
+9\end{array}$ & $\begin{array}{l}\text { IV } \\
\text { IV } \\
\text { IV }\end{array}$ & $\begin{array}{l}d \\
b \\
b\end{array}$ \\
\hline $\begin{array}{r}48.660 \\
52.170 \\
56.475 \\
64.795 \\
4673.174\end{array}$ & $\begin{array}{l}\mathrm{B} \\
\mathrm{A} \\
\mathrm{A} \\
\mathrm{B} \\
\mathrm{C}\end{array}$ & $\begin{array}{r}10 \\
16 \\
22 \\
17 \\
7\end{array}$ & $\begin{array}{l}.835 \mathrm{~s} \\
.343 \\
.644 \\
.965 \\
.347\end{array}$ & $\begin{array}{l}\mathrm{Ni} \\
\mathrm{Cr} \\
\mathrm{Ti} \\
\mathrm{Cr} \\
\mathrm{Fe}\end{array}$ & $\begin{array}{l}4 \\
5 \\
3 \\
3 \\
4 \\
4\end{array}$ & $\begin{array}{l}175 \\
173 \\
169 \\
170 \\
173\end{array}$ & $\begin{array}{l}173 \\
172 \\
172 \\
172 \\
173\end{array}$ & $\begin{array}{l}+7 \\
+11\end{array}$ & $\begin{array}{l}\text { III } \\
\text { I } \\
\text { I } \\
\text { III }\end{array}$ & \\
\hline
\end{tabular}

6 4447.7. One series of measurements gives .730 for the fractional part of this wave length. The results from many o ther plates are in good agreement and give the value tabulated. A companion affects the wave length as measured by the interferometer. 
TABLE 1.-A. O. B.S. solar wave lengths-Continued

\begin{tabular}{|c|c|c|c|c|c|c|c|c|c|c|}
\hline \multirow[t]{2}{*}{ A. O.B.S. $\lambda$} & \multirow{2}{*}{$\begin{array}{l}\text { Proba- } \\
\text { ble } \\
\text { elror }\end{array}$} & \multirow{2}{*}{$\begin{array}{l}\text { No. } \\
\text { obs. }\end{array}$} & \multicolumn{3}{|c|}{ Rowland } & \multicolumn{2}{|c|}{$\begin{array}{l}\text { Correction to } \\
\text { Rowland }\end{array}$} & \multirow[t]{2}{*}{ Sun-are } & \multirow{2}{*}{\multicolumn{2}{|c|}{$\begin{array}{l}\text { Tempera- } \\
\text { ture and } \\
\text { pressure } \\
\text { classes }\end{array}$}} \\
\hline & & & $\lambda$ & Iden. & Int. & Obs. & Mean & & & \\
\hline $\begin{array}{r}4678.171 \\
78.855 \\
83.567 \\
86.221 \\
4690.146\end{array}$ & $\begin{array}{l}\mathrm{B} \\
\mathrm{B} \\
\mathrm{A} \\
\mathrm{A} \\
\mathrm{A}\end{array}$ & $\begin{array}{l}17 \\
19 \\
22 \\
20 \\
22\end{array}$ & $\begin{array}{r}.347 \mathrm{~s} \\
9.027 \mathrm{~s} \\
.745 \mathrm{~s} \\
.395 \mathrm{~s} \\
.317 \mathrm{~s}\end{array}$ & $\begin{array}{l}\mathrm{Cd} \\
\mathrm{Fe} \\
\mathrm{Fe} \\
\mathrm{Ni} \\
\mathrm{Fe} ?\end{array}$ & $\begin{array}{c}3 \mathrm{~N} \\
6 \\
3 \\
3 \\
4\end{array}$ & $\begin{array}{l}176 \\
172 \\
178 \\
174 \\
173\end{array}$ & $\begin{array}{l}173 \\
173 \\
173 \\
173 \\
173\end{array}$ & $\begin{array}{l}+9 \\
+4 \\
+8\end{array}$ & $\begin{array}{l}\mathrm{V} \\
\text { III }\end{array}$ & \\
\hline $\begin{array}{r}400.164 \\
03.006 \\
03.817 \\
04.956 \\
09.718\end{array}$ & $\begin{array}{l}\mathrm{A} \\
\mathrm{B} \\
\mathrm{C} \\
\mathrm{A} \\
\mathrm{A}\end{array}$ & $\begin{array}{l}15 \\
11 \\
11 \\
20 \\
13\end{array}$ & $\begin{array}{c}.337 \\
.177 \mathrm{~s} \\
.994 \mathrm{~s} \\
5.131 \\
.896\end{array}$ & $\begin{array}{c}(\mathrm{Fe}) \\
\mathrm{Mg} \\
\mathrm{Ni} \\
\mathrm{Fe} \\
\mathrm{Mn}\end{array}$ & $\begin{array}{c}4 \\
10 \\
3 \\
4 \\
2\end{array}$ & $\begin{array}{l}173 \\
171 \\
177 \\
175 \\
178\end{array}$ & $\begin{array}{l}174 \\
174 \\
174 \\
174 \\
175\end{array}$ & $\begin{array}{l}+7 \\
+9\end{array}$ & $\mathrm{v}$ & d \\
\hline $\begin{array}{l}15.774 \\
18.428 \\
21.001 \\
22.166 \\
28.554\end{array}$ & $\begin{array}{l}\text { B } \\
\text { B } \\
\text { C } \\
\text { A } \\
\text { B }\end{array}$ & $\begin{array}{r}13 \\
13 \\
5 \\
22 \\
11\end{array}$ & $\begin{array}{l}.946 \\
.601 \\
.179 \\
.342 \mathrm{~s} \\
.732\end{array}$ & $\begin{array}{l}\mathrm{Ni} \\
\mathrm{Cr} \\
\mathrm{Fe} ? \\
\mathrm{Zn} \\
\mathrm{Fe}\end{array}$ & $\begin{array}{l}4 \\
3 \\
2 \\
3 \\
4\end{array}$ & $\begin{array}{l}172 \\
173 \\
178 \\
176 \\
178\end{array}$ & $\begin{array}{l}175 \\
175 \\
175 \\
176 \\
176\end{array}$ & +7 & III & \\
\hline $\begin{array}{l}33.602 \\
36.785 \\
41.537 \\
45.808 \\
52.431\end{array}$ & $\begin{array}{l}\mathrm{A} \\
\mathrm{C} \\
\mathrm{A} \\
\mathrm{B} \\
\mathrm{C}\end{array}$ & $\begin{array}{r}19 \\
14 \\
18 \\
17 \\
8\end{array}$ & $\begin{array}{l}.779 \\
.963 \\
.718 \\
.992 \\
.613\end{array}$ & $\begin{array}{l}\mathrm{Fe} \\
\mathrm{Fe} \\
\mathrm{Fe} \\
\mathrm{Fe} \\
\mathrm{Ni}\end{array}$ & $\begin{array}{l}4 \\
6 \\
3 \\
4 \\
3\end{array}$ & $\begin{array}{l}177 \\
178 \\
181 \\
184 \\
182\end{array}$ & $\begin{array}{l}177 \\
178 \\
179 \\
180 \\
181\end{array}$ & $\begin{array}{l}+11 \\
+12 \\
+6 \\
+7\end{array}$ & $\begin{array}{l}\text { IB } \\
\text { II } \\
\text { V } \\
\text { V }\end{array}$ & $\begin{array}{l}\mathrm{a} \\
\mathrm{d} \\
\mathrm{b} \\
\mathrm{b}\end{array}$ \\
\hline $\begin{array}{r}54.047 \\
4761.534 \\
66.430 \\
72.826 \\
79.985\end{array}$ & $\begin{array}{l}\text { C } \\
\text { B } \\
\text { B } \\
\text { C } \\
\text { C }\end{array}$ & $\begin{array}{r}12 \\
9 \\
9 \\
8 \\
10\end{array}$ & $\begin{array}{c}.225 \mathrm{~s} \\
.718 \\
.621 \\
3.007 \\
.169\end{array}$ & $\begin{array}{l}\mathrm{Mn} \\
\mathrm{Mn} \\
\mathrm{Mn} \\
\mathrm{Fe} \\
\mathrm{Co}\end{array}$ & $\begin{array}{l}7 \\
3 \\
4 \\
4 \\
2\end{array}$ & $\begin{array}{l}178 \\
184 \\
181 \\
181 \\
184\end{array}$ & $\begin{array}{l}182 \\
183 \\
183 \\
183 \\
184\end{array}$ & +10 & $\begin{array}{l}\text { I } \\
\text { III } \\
\text { III } \\
\text { IIII }\end{array}$ & $\mathrm{b}$ \\
\hline $\begin{array}{r}86.820 \\
88.766 \\
4789.659 \\
4802.838 \\
10.539\end{array}$ & $\begin{array}{l}\text { C } \\
\text { A } \\
\text { C } \\
\text { A } \\
\text { B }\end{array}$ & $\begin{array}{r}8 \\
10 \\
11 \\
10 \\
7\end{array}$ & $\begin{array}{c}7.003 \\
.952 \\
.849 \\
3.072 \\
.724 \mathrm{~s}\end{array}$ & $\begin{array}{l}\mathrm{Fe} \\
\mathrm{Fe} \\
\mathrm{Fe} \\
\mathrm{Fe} \\
\mathrm{Zn}\end{array}$ & $\begin{array}{l}2 \\
3 \\
3 \\
2 \\
3\end{array}$ & $\begin{array}{l}183 \\
186 \\
190 \\
184 \\
185\end{array}$ & $\begin{array}{l}185 \\
185 \\
185 \\
185 \\
185\end{array}$ & $\begin{array}{l}+14 \\
+9 \\
+9 \\
+6\end{array}$ & $\begin{array}{l}\text { IV? } \\
\text { V }\end{array}$ & b \\
\hline $\begin{array}{l}23.516 \\
24.145 \\
32.718 \\
39.551 \\
48.257\end{array}$ & $\begin{array}{l}\mathrm{B} \\
\mathrm{D} \\
\mathrm{B} \\
\mathrm{B} \\
\mathrm{D}\end{array}$ & $\begin{array}{l}7 \\
8 \\
9 \\
9 \\
8\end{array}$ & $\begin{array}{l}.697 \mathrm{~s} \\
.325 \mathrm{~s} \\
.905 \\
.734 \\
.438\end{array}$ & $\begin{array}{l}\mathrm{Mn} \\
\mathrm{Fe} \\
\mathrm{Fe} \\
\mathrm{Fe}\end{array}$ & $\begin{array}{l}5 \\
3 \\
3 \\
3 \\
2\end{array}$ & $\begin{array}{l}181 \\
180 \\
187 \\
183 \\
181\end{array}$ & $\begin{array}{l}184 \\
184 \\
183 \\
182 \\
181\end{array}$ & $\begin{array}{l}+7 \\
+6\end{array}$ & I & \\
\hline $\begin{array}{l}54.875 \\
59.751 \\
70.824 \\
82.154 \\
85.437\end{array}$ & $\begin{array}{l}\mathrm{A} \\
\mathrm{B} \\
\mathrm{B} \\
\mathrm{D} \\
\mathrm{D}\end{array}$ & $\begin{array}{l}6 \\
5 \\
7 \\
9 \\
7\end{array}$ & $\begin{array}{l}5.059 \\
.928 \mathrm{~s} \\
.996 \\
.336 \\
.620\end{array}$ & $\begin{array}{c}\mathrm{Fe} \\
\mathrm{Fe} \\
\mathrm{Ni}, \mathrm{Cr} \\
\mathrm{Fe} \\
\mathrm{Fe}\end{array}$ & $\begin{array}{l}1 \\
4 \\
3 \\
3 \\
3\end{array}$ & $\begin{array}{l}184 \\
177 \\
172 \\
182 \\
183\end{array}$ & $\begin{array}{l}179 \\
179 \\
177 \\
176 \\
175\end{array}$ & $\begin{array}{l}+10 \\
+9 \\
+7\end{array}$ & $\begin{array}{l}\text { III } \\
\mathrm{V}\end{array}$ & $\begin{array}{l}\mathrm{e} 5 \\
\mathrm{~d}\end{array}$ \\
\hline $\begin{array}{r}4892.867 \\
4904.424 \\
17.239 \\
24.784 \\
38.184\end{array}$ & $\begin{array}{l}\mathrm{B} \\
\mathrm{C} \\
\mathrm{A} \\
\mathrm{A} \\
\mathrm{A}\end{array}$ & $\begin{array}{r}8 \\
12 \\
10 \\
12 \\
7\end{array}$ & $\begin{array}{l}3.030 \\
.597 \\
.410 \\
.956 \mathrm{~s} \\
.350\end{array}$ & $\begin{array}{l}\mathrm{Fe} \\
\mathrm{Fe} \\
\mathrm{Fe} \\
\mathrm{Fe}\end{array}$ & $\begin{array}{l}1 \\
3 \\
2 \\
3 \\
2\end{array}$ & $\begin{array}{l}163 \\
173 \\
171 \\
172 \\
166\end{array}$ & $\begin{array}{l}175 \\
173 \\
172 \\
171 \\
171\end{array}$ & $\begin{array}{l}+11 \\
+10\end{array}$ & V & b \\
\hline $\begin{array}{l}38.825 \\
39.697 \\
46.401 \\
50.117 \\
53.217\end{array}$ & $\begin{array}{l}\mathrm{D} \\
\mathrm{B} \\
\mathrm{B} \\
\mathrm{B} \\
\mathrm{C}\end{array}$ & $\begin{array}{r}7 \\
13 \\
10 \\
13 \\
8\end{array}$ & $\begin{array}{l}. .997 \\
.868 \\
.568 \\
.291 \\
.392\end{array}$ & $\begin{array}{l}\mathrm{Fe} \\
\mathrm{Fe} \\
\mathrm{Fe} \\
\mathrm{Fe} \\
\mathrm{Ni}\end{array}$ & $\begin{array}{l}4 \\
3 \\
3 \\
2 \\
2\end{array}$ & $\begin{array}{l}172 \\
171 \\
167 \\
174 \\
175\end{array}$ & $\begin{array}{l}171 \\
171 \\
171 \\
171 \\
171\end{array}$ & $\begin{array}{l}+13 \\
+11 \\
+14 \\
+11\end{array}$ & $\begin{array}{l}\text { IV } \\
\text { IB } \\
\text { IV } \\
\text { V? }\end{array}$ & $\begin{array}{l}\mathrm{d} \\
\mathrm{a} \\
\mathrm{d} \\
\mathrm{d}\end{array}$ \\
\hline $\begin{array}{l}62.583 \\
66.102 \\
67.909 \\
73.110 \\
83.262\end{array}$ & $\begin{array}{l}\text { B } \\
\text { B } \\
\text { C } \\
\text { C } \\
\text { B }\end{array}$ & $\begin{array}{l}12 \\
12 \\
14 \\
12 \\
14\end{array}$ & $\begin{array}{c}.751 \\
.270 \\
8.080 \\
.281 \mathrm{~s} \\
.433\end{array}$ & $\begin{array}{c}\mathrm{Fe} \\
\mathrm{Fe} \\
\mathrm{Fe} \\
\mathrm{Ti}, \mathrm{Fe} \\
\mathrm{Fe}\end{array}$ & $\begin{array}{l}2 \\
4 \\
3 \\
4 \\
3\end{array}$ & $\begin{array}{l}168 \\
168 \\
171 \\
171 \\
171\end{array}$ & $\begin{array}{l}171 \\
171 \\
171 \\
171 \\
171\end{array}$ & $\begin{array}{l}+13 \\
+10 \\
+14\end{array}$ & $\begin{array}{l}\mathrm{V} \\
\mathrm{V} \\
\mathrm{V}\end{array}$ & $\begin{array}{l}\text { d } \\
\text { e } \\
d\end{array}$ \\
\hline $\begin{array}{r}4994.140 \\
5001.876 \\
02.802 \\
14.954 \\
28.135\end{array}$ & $\begin{array}{l}\mathrm{B} \\
\mathrm{C} \\
\mathrm{A} \\
\mathrm{C} \\
\mathrm{A}\end{array}$ & $\begin{array}{l}16 \\
15 \\
19 \\
14 \\
17\end{array}$ & $\begin{array}{c}.316 \mathrm{~s} \\
2.044 \\
.976 \\
5.123 \\
.308\end{array}$ & $\begin{array}{l}\mathrm{Fe} \\
\mathrm{Fe} \\
\mathrm{Fe} \\
\mathrm{Fe} \\
\mathrm{Fe}\end{array}$ & $\begin{array}{l}3 \\
5 \\
2 \\
3 \\
2\end{array}$ & $\begin{array}{l}176 \\
168 \\
174 \\
169 \\
173\end{array}$ & $\begin{array}{l}171 \\
171 \\
171 \\
171 \\
171\end{array}$ & $\begin{array}{r}+10 \\
+12 \\
+10 \\
+12 \\
+8\end{array}$ & $\begin{array}{l}\text { IB } \\
\text { V } \\
\text { V }\end{array}$ & $\begin{array}{l}\mathrm{a} \\
\mathrm{d} \\
\mathrm{d}\end{array}$ \\
\hline $\begin{array}{r}39.968 \\
48.448 \\
49.834 \\
60.080 \\
5067.160\end{array}$ & $\begin{array}{l}\mathrm{B} \\
\mathrm{A} \\
\mathrm{B} \\
\mathrm{A} \\
\mathrm{B}\end{array}$ & $\begin{array}{l}20 \\
13 \\
13 \\
21 \\
20\end{array}$ & $\begin{array}{l}0.138 \\
.612 \\
0.008 \mathrm{~s} \\
.258 \mathrm{~s} \\
.336\end{array}$ & $\begin{array}{l}\mathrm{Ti} \\
\mathrm{Fe} \\
\mathrm{Fe} \\
\mathrm{Fe} \\
\mathrm{Fe}\end{array}$ & $\begin{array}{l}3 \\
3 \\
6 \\
3 \\
3\end{array}$ & $\begin{array}{l}170 \\
164 \\
174 \\
178 \\
176\end{array}$ & $\begin{array}{l}171 \\
171 \\
171 \\
170 \\
170\end{array}$ & $\begin{array}{l}+9 \\
+15\end{array}$ & $\begin{array}{l}\text { I } \\
\text { III }\end{array}$ & $\begin{array}{l}a \\
a\end{array}$ \\
\hline
\end{tabular}


TABLE 1.-A. O. B. S. solar wave lengths-Continued

\begin{tabular}{|c|c|c|c|c|c|c|c|c|c|c|}
\hline \multirow{2}{*}{ 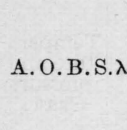 } & \multirow{2}{*}{$\begin{array}{l}\text { Proba- } \\
\text { ble } \\
\text { error }\end{array}$} & \multirow{2}{*}{$\begin{array}{l}\text { No. } \\
\text { obs. }\end{array}$} & \multicolumn{3}{|c|}{ Rowland } & \multicolumn{2}{|c|}{$\begin{array}{l}\text { Correction to } \\
\text { Rowland }\end{array}$} & \multirow{2}{*}{ Sun-are } & \multirow{2}{*}{\multicolumn{2}{|c|}{$\begin{array}{l}\text { Tempera } \\
\text { ture and } \\
\text { pressure } \\
\text { classes }\end{array}$}} \\
\hline & & & $\lambda$ & Iden. & Int. & Obs. & Mean & & & \\
\hline $\begin{array}{r}5068.779 \\
74.762 \\
76.278 \\
79.241 \\
79.749\end{array}$ & $\begin{array}{l}\mathrm{C} \\
\mathrm{C} \\
\mathrm{B} \\
\mathrm{C} \\
\mathrm{B}\end{array}$ & $\begin{array}{l}15 \\
15 \\
15 \\
14 \\
15\end{array}$ & $\begin{array}{l}.944 \mathrm{~s} \\
.932 \\
.450 \\
.409 \\
.921\end{array}$ & $\begin{array}{l}\mathrm{Fe} \\
\mathrm{Fe} \\
\mathrm{Fe} \\
\mathrm{Fe} \\
\mathrm{Fe}\end{array}$ & $\begin{array}{l}5 \\
5 \\
3 \\
4 \\
4\end{array}$ & $\begin{array}{l}165 \\
170 \\
172 \\
168 \\
172\end{array}$ & $\begin{array}{l}169 \\
169 \\
169 \\
169 \\
169\end{array}$ & $\begin{array}{l}+13 \\
+14 \\
+8\end{array}$ & $\begin{array}{l}\text { V } \\
\text { V } \\
\text { IV } \\
\text { IB }\end{array}$ & $\begin{array}{l}d \\
e \\
b \\
a\end{array}$ \\
\hline $\begin{array}{r}83.351 \\
90.787 \\
5099.941 \\
5109.661 \\
15.401\end{array}$ & $\begin{array}{l}\mathrm{A} \\
\mathrm{A} \\
\mathrm{A} \\
\mathrm{B} \\
\mathrm{B}\end{array}$ & $\begin{array}{l}21 \\
18 \\
21 \\
17 \\
16\end{array}$ & $\begin{array}{r}.518 \mathrm{~s} \\
.954 \mathrm{~s} \\
0.108 \\
.827 \mathrm{~s} \\
.566\end{array}$ & $\begin{array}{l}\mathrm{Fe} \\
\mathrm{Fe} \\
\mathrm{Ni} \\
\mathrm{Fe} \\
\mathrm{Ni}\end{array}$ & $\begin{array}{l}4 \\
5 \\
2 \\
2 \\
2\end{array}$ & $\begin{array}{l}167 \\
167 \\
167 \\
166 \\
165\end{array}$ & $\begin{array}{l}168 \\
168 \\
167 \\
167 \\
166\end{array}$ & $\begin{array}{l}+13 \\
+12\end{array}$ & $\begin{array}{l}\text { IB } \\
\mathrm{V}\end{array}$ & a \\
\hline $\begin{array}{l}26.204 \\
37.394 \\
50.852 \\
59.067 \\
73.752\end{array}$ & $\begin{array}{l}\text { C } \\
\text { B } \\
\text { C } \\
\text { B } \\
\text { A }\end{array}$ & $\begin{array}{l}18 \\
16 \\
17 \\
19 \\
21\end{array}$ & $\begin{array}{r}.371 \mathrm{~s} \\
.588 \\
1.020 \mathrm{~s} \\
.231 \mathrm{~s} \\
.917 \mathrm{~s}\end{array}$ & $\begin{array}{c}\mathrm{Fe}, \mathrm{Co} \\
\mathrm{Fe} \\
\mathrm{Fe} \\
\mathrm{Fe} \\
\mathrm{Ti}\end{array}$ & $\begin{array}{l}2 \\
3 \\
4 \\
2 \\
2\end{array}$ & $\begin{array}{l}167 \\
164 \\
168 \\
164 \\
165\end{array}$ & $\begin{array}{l}166 \\
165 \\
165 \\
165 \\
164\end{array}$ & $\begin{array}{l}+12 \\
+12 \\
+10\end{array}$ & $\begin{array}{l}\text { V } \\
\text { IB } \\
I\end{array}$ & \\
\hline $\begin{array}{r}85.911 \\
5198.723 \\
5210.396 \\
17.403 \\
25.537\end{array}$ & $\begin{array}{l}\mathrm{A} \\
\mathrm{B} \\
\mathrm{A} \\
\mathrm{B} \\
\mathrm{A}\end{array}$ & $\begin{array}{l}20 \\
21 \\
23 \\
21 \\
23\end{array}$ & $\begin{array}{l}6.073 \\
.888 \mathrm{~s} \\
.555 \mathrm{~s} \\
.552 \mathrm{~s} \\
.695 \mathrm{~s}\end{array}$ & $\begin{array}{l}\mathrm{Ti} \\
\mathrm{Fe} \\
\mathrm{Ti} \\
\mathrm{Fe} \\
\mathrm{Fe}\end{array}$ & $\begin{array}{l}2 \\
3 \\
3 \\
3 \\
2\end{array}$ & $\begin{array}{l}162 \\
165 \\
169 \\
149 \\
158\end{array}$ & $\begin{array}{l}163 \\
162 \\
161 \\
155\end{array}$ & $\begin{array}{l}+11 \\
+10 \\
+15\end{array}$ & $\begin{array}{l}\text { IV } \\
\text { I } \\
\text { V } \\
\text { IA }\end{array}$ & $\begin{array}{l}\mathrm{a} \\
\mathrm{a} \\
\mathrm{d}\end{array}$ \\
\hline $\begin{array}{r}42.502 \\
53.471 \\
63.318 \\
73.391 \\
5288.536\end{array}$ & $\begin{array}{l}\text { A } \\
\text { A } \\
\text { A } \\
\text { C } \\
\text { A }\end{array}$ & $\begin{array}{l}22 \\
24 \\
23 \\
14 \\
24\end{array}$ & $\begin{array}{l}.658 \mathrm{~s} \\
.633 \mathrm{~s} \\
.486 \\
.558 \mathrm{~s} \\
.705 \mathrm{~s}\end{array}$ & $\begin{array}{c}\mathrm{Fe} \\
\mathrm{Fe} \\
\mathrm{Fe} \\
\mathrm{Fe}, \mathrm{Cr} \\
\mathrm{Fe}\end{array}$ & $\begin{array}{l}2 \\
2 \\
4 \\
2 \\
2\end{array}$ & $\begin{array}{l}156 \\
162 \\
168 \\
167 \\
169\end{array}$ & $\begin{array}{l}159 \\
162 \\
165 \\
167 \\
170\end{array}$ & $\begin{array}{l}+12 \\
+13\end{array}$ & $\begin{array}{l}\text { IV } \\
\text { V }\end{array}$ & $\begin{array}{l}\mathrm{a} \\
\mathrm{d}\end{array}$ \\
\hline $\begin{array}{r}5300.753 \\
07.370 \\
22.051 \\
32.909 \\
48.327\end{array}$ & $\begin{array}{l}\mathrm{A} \\
\mathrm{A} \\
\mathrm{A} \\
\mathrm{A} \\
\mathrm{A}\end{array}$ & $\begin{array}{l}22 \\
23 \\
14 \\
13 \\
16\end{array}$ & $\begin{array}{l}.929 \mathrm{~s} \\
.5411 \mathrm{~s} \\
.227 \\
3.089 \mathrm{~s} \\
.511\end{array}$ & $\begin{array}{l}\mathrm{Cr} \\
\mathrm{Fe} \\
\mathrm{Fe} \\
\mathrm{Fe} \\
\mathrm{Cr}\end{array}$ & $\begin{array}{l}2 \\
3 \\
3 \\
4 \\
4\end{array}$ & $\begin{array}{l}176 \\
171 \\
176 \\
180 \\
184\end{array}$ & $\begin{array}{l}172 \\
173 \\
177 \\
179 \\
183\end{array}$ & $\begin{array}{l}+10 \\
+8\end{array}$ & $\begin{array}{l}\text { I } \\
\text { III? } \\
\text { IB? } \\
\text { I }\end{array}$ & \\
\hline $\begin{array}{r}65.409 \\
79.583 \\
89.487 \\
5398.290 \\
5409.799\end{array}$ & $\begin{array}{l}\text { A } \\
\text { A } \\
\text { A } \\
\text { A } \\
\text { A }\end{array}$ & $\begin{array}{l}16 \\
14 \\
14 \\
14 \\
14\end{array}$ & $\begin{array}{r}.596 \\
.775 \mathrm{~s} \\
.683 \mathrm{~s} \\
.486 \\
0.000 \mathrm{~s}\end{array}$ & $\begin{array}{l}\mathrm{Fe} \\
\mathrm{Fe} \\
\mathrm{Fe} \\
\mathrm{Fe} \\
\mathrm{Cr}\end{array}$ & $\begin{array}{l}3 \\
3 \\
3 \\
3 \\
4 \\
4\end{array}$ & $\begin{array}{l}187 \\
192 \\
196 \\
196 \\
201\end{array}$ & $\begin{array}{l}187 \\
192 \\
195 \\
197 \\
201\end{array}$ & +7 & V & $\begin{array}{l}a \\
a\end{array}$ \\
\hline $\begin{array}{r}15.215 \\
5432956 \\
45.055 \\
62.971 \\
73.909\end{array}$ & $\begin{array}{l}\text { C } \\
\text { B } \\
\text { A } \\
\text { A } \\
\text { A }\end{array}$ & $\begin{array}{l}11 \\
14 \\
16 \\
15 \\
18\end{array}$ & $\begin{array}{l}.416 \mathrm{~s} \\
\text { 3.160 } \\
.259 \\
\text { 3.174 } \\
4.113\end{array}$ & $\begin{array}{c}\mathrm{Fe}, \mathrm{V} \\
\mathrm{Fe} \\
\mathrm{Fe} \\
\mathrm{Fe} \\
\mathrm{Fe}\end{array}$ & $\begin{array}{l}5 \\
2 \\
4 \\
3 \\
3\end{array}$ & $\begin{array}{l}201 \\
204 \\
204 \\
203 \\
204\end{array}$ & $\begin{array}{l}202 \\
203 \\
204 \\
204 \\
204\end{array}$ & +13 & V & $\begin{array}{l}e \\
e \\
d\end{array}$ \\
\hline $\begin{array}{r}5487.754 \\
5501.477 \\
12.989 \\
25.552 \\
34.848\end{array}$ & $\begin{array}{l}\mathrm{B} \\
\mathrm{A} \\
\mathrm{B} \\
\mathrm{A} \\
\mathrm{A}\end{array}$ & $\begin{array}{l}19 \\
18 \\
18 \\
17 \\
22\end{array}$ & $\begin{array}{r}.959 \mathrm{~s} \\
.683 \mathrm{~s} \\
3.198 \mathrm{~s} \\
.765 \\
5.061 \mathrm{~s}\end{array}$ & $\begin{array}{l}\mathrm{Fe} \\
\mathrm{Fe} \\
\mathrm{Ca} \\
\mathrm{Fe} \\
\mathrm{Fe}\end{array}$ & $\begin{array}{l}3 \\
5 \\
4 \\
2 \\
2\end{array}$ & $\begin{array}{l}205 \\
206 \\
209 \\
213 \\
213\end{array}$ & $\begin{array}{l}205 \\
206 \\
209 \\
212 \\
214\end{array}$ & +7 & $\begin{array}{l}\text { IB } \\
\text { III }\end{array}$ & a3 \\
\hline $\begin{array}{r}46.516 \\
60.217 \\
76.104 \\
5590.125 \\
5601.286\end{array}$ & $\begin{array}{l}\text { B } \\
\text { A } \\
\text { A } \\
\text { A } \\
\text { A }\end{array}$ & $\begin{array}{l}20 \\
24 \\
24 \\
24 \\
22\end{array}$ & $\begin{array}{l}.732 \\
434 \\
.320 \mathrm{~s} \\
.343 \mathrm{~s} \\
.505 \mathrm{~s}\end{array}$ & $\begin{array}{l}\mathrm{Fe} \\
\mathrm{Fe} \\
\mathrm{Fe} \\
\mathrm{Ca} \\
\mathrm{Ca}\end{array}$ & $\begin{array}{l}2 \\
2 \\
4 \\
3 \\
3\end{array}$ & $\begin{array}{l}216 \\
217 \\
216 \\
218 \\
217\end{array}$ & $\begin{array}{l}216 \\
216 \\
217 \\
217 \\
217\end{array}$ & & $\begin{array}{l}\text { IV } \\
\text { III } \\
\text { III }\end{array}$ & $\mathrm{d} 5$ \\
\hline $\begin{array}{l}18.640 \\
24.558 \\
41.447 \\
55.499 \\
67.525\end{array}$ & $\begin{array}{l}\mathrm{A} \\
\mathrm{A} \\
\mathrm{A} \\
\mathrm{B} \\
\mathrm{B}\end{array}$ & $\begin{array}{l}19 \\
20 \\
18 \\
18 \\
15\end{array}$ & $\begin{array}{l}.858 \\
.769 \mathrm{~s} \\
.667 \mathrm{~s} \\
.715 \mathrm{~s} \\
.739\end{array}$ & $\begin{array}{c}\mathrm{Fe} \\
\mathrm{Fe}, \mathrm{V} \\
\mathrm{Fe} \\
\mathrm{Fe} \\
\mathrm{Fe}\end{array}$ & $\begin{array}{l}1 \\
4 \\
2 \\
2 \\
2\end{array}$ & $\begin{array}{l}218 \\
211 \\
220 \\
216 \\
214\end{array}$ & $\begin{array}{l}218 \\
218 \\
218 \\
217 \\
216\end{array}$ & & $\begin{array}{l}\text { IV } \\
\text { V }\end{array}$ & \\
\hline $\begin{array}{r}79.032 \\
5690.433 \\
5701.557 \\
17.839 \\
31.772\end{array}$ & $\begin{array}{l}\mathrm{A} \\
\mathrm{A} \\
\mathrm{A} \\
\mathrm{A} \\
\mathrm{A}\end{array}$ & $\begin{array}{l}17 \\
15 \\
17 \\
16 \\
14\end{array}$ & $\begin{array}{c}.249 \mathrm{~s} \\
.646 \\
.772 \mathrm{~s} \\
8.055 \\
.984 \mathrm{~s}\end{array}$ & $\begin{array}{l}\mathrm{Fe} \\
\mathrm{Si} \\
\mathrm{Fe} \\
\mathrm{Fe} \\
\mathrm{Fe}\end{array}$ & $\begin{array}{l}3 \\
3 \\
4 \\
4 \\
4\end{array}$ & $\begin{array}{l}217 \\
213 \\
215 \\
216 \\
212\end{array}$ & $\begin{array}{l}216 \\
215 \\
214 \\
214 \\
213\end{array}$ & +9 & III? & \\
\hline $\begin{array}{r}41.856 \\
52.042 \\
600.841 \\
72.156 \\
5783.871\end{array}$ & $\begin{array}{l}\mathrm{C} \\
\mathrm{C} \\
\mathrm{B} \\
\mathrm{C} \\
\mathrm{C}\end{array}$ & $\begin{array}{r}9 \\
13 \\
10 \\
10 \\
11\end{array}$ & $\begin{array}{r}2.068 \mathrm{~s} \\
.254 \mathrm{~s} \\
1.052 \\
.364 \mathrm{~s} \\
4.080 \mathrm{~s}\end{array}$ & $\begin{array}{l}\mathrm{Fe} \\
\mathrm{Fe} \\
\mathrm{Ni} \\
\mathrm{Si} \\
\mathrm{Cr}\end{array}$ & $\begin{array}{l}2 \\
4 \\
2 \\
3 \\
3\end{array}$ & $\begin{array}{l}212 \\
212 \\
211 \\
208 \\
209\end{array}$ & $\begin{array}{l}212 \\
211 \\
211 \\
210 \\
209\end{array}$ & & $\begin{array}{l}\text { IV } \\
\text { III }\end{array}$ & \\
\hline
\end{tabular}


TABle 1.-A. O. B. S. solar wave lengths-Continued

\begin{tabular}{|c|c|c|c|c|c|c|c|c|c|}
\hline \multirow{2}{*}{ A. O.B.S. ג } & \multirow{2}{*}{$\begin{array}{l}\text { Proba- } \\
\text { ble } \\
\text { error }\end{array}$} & \multirow{2}{*}{$\begin{array}{l}\text { No. } \\
\text { obs. }\end{array}$} & \multicolumn{3}{|c|}{ Rowland } & \multicolumn{2}{|c|}{$\begin{array}{l}\text { Correction to } \\
\text { Rowland }\end{array}$} & \multirow{2}{*}{ Sun-are } & \multirow{2}{*}{$\begin{array}{l}\text { Tempera- } \\
\text { ture and } \\
\text { pressure } \\
\text { classes }\end{array}$} \\
\hline & & & $\lambda$ & Iden. & Int. & Obs. & Mean & & \\
\hline $\begin{array}{r}5797.870 \\
5805.226 \\
06.738 \\
09.227 \\
16.379\end{array}$ & $\begin{array}{l}\mathrm{B} \\
\mathrm{C} \\
\mathrm{D} \\
\mathrm{B} \\
\mathrm{C}\end{array}$ & $\begin{array}{r}9 \\
6 \\
6 \\
19 \\
6\end{array}$ & $\begin{array}{r}8.077 \mathrm{~s} \\
.441 \mathrm{~s} \\
.950 \mathrm{~s} \\
.439 \mathrm{~s} \\
.601 \mathrm{~s}\end{array}$ & $\begin{array}{l}\mathrm{Ni} \\
\mathrm{Fe} \\
\mathrm{Fe} \\
\mathrm{Fe}\end{array}$ & $\begin{array}{l}3 \\
4 \\
5 \\
4 \\
5\end{array}$ & $\begin{array}{l}207 \\
215 \\
212 \\
212 \\
222\end{array}$ & $\begin{array}{l}209 \\
214 \\
214 \\
214 \\
214\end{array}$ & \pm 0 & V \\
\hline $\begin{array}{l}47.012 \\
48.129 \\
52.235 \\
53.688 \\
55.087\end{array}$ & $\begin{array}{l}\mathrm{D} \\
\mathrm{C} \\
\mathrm{D} \\
\mathrm{A} \\
\mathrm{D}\end{array}$ & $\begin{array}{r}4 \\
14 \\
7 \\
17 \\
4\end{array}$ & $\begin{array}{l}.221 \\
.342 \\
.443 \\
.902 \mathrm{~s} \\
.300\end{array}$ & $\begin{array}{l}\mathrm{Ni} \\
\mathrm{Fe} \\
\mathrm{Fe} \\
\mathrm{Ba} \\
\mathrm{Fe}\end{array}$ & $\begin{array}{l}1 \\
3 \\
3 \\
5 \\
1\end{array}$ & $\begin{array}{l}209 \\
213 \\
208 \\
214 \\
213\end{array}$ & $\begin{array}{l}213 \\
213 \\
213 \\
213 \\
213\end{array}$ & +9 & III \\
\hline $\begin{array}{l}56.101 \\
57.459 \\
57.762 \\
59.596 \\
62.368\end{array}$ & $\begin{array}{l}\mathrm{B} \\
\mathrm{B} \\
\mathrm{C} \\
\mathrm{A} \\
\mathrm{B}\end{array}$ & $\begin{array}{r}8 \\
12 \\
6 \\
25 \\
15\end{array}$ & $\begin{array}{l}.312 \\
.674 \mathrm{~s} \\
.976 \\
.809 \mathrm{~s} \\
.582 \mathrm{~s}\end{array}$ & $\begin{array}{l}\mathrm{Fe} \\
\mathrm{Ca} \\
\mathrm{Ni} \\
\mathrm{Fe} \\
\mathrm{Fe}\end{array}$ & $\begin{array}{l}2 \\
8 \\
3 \\
5 \\
6\end{array}$ & $\begin{array}{l}211 \\
215 \\
214 \\
213 \\
214\end{array}$ & $\begin{array}{l}213 \\
213 \\
213 \\
213 \\
213\end{array}$ & $\begin{array}{l}+8 \\
+3 \\
+11\end{array}$ & $\begin{array}{l}\text { III } \\
\text { IV } \\
\text { V } \\
\text { V }\end{array}$ \\
\hline $\begin{array}{l}66.461 \\
67.573 \\
85.978 \\
87.217 \\
91.656\end{array}$ & $\begin{array}{l}\mathrm{A} \\
\mathrm{B} \\
\mathrm{D} \\
\mathrm{C} \\
\mathrm{B}\end{array}$ & $\begin{array}{r}23 \\
6 \\
7 \\
4 \\
4\end{array}$ & $\begin{array}{r}.675 \\
.785 \\
6.193 \\
.445 \\
.878\end{array}$ & $\begin{array}{l}\mathrm{Ti} \\
\mathrm{Ca} \\
\text { Air } \\
\text { Air } \\
\text { Air }\end{array}$ & $\begin{array}{l}3 \\
2 \\
5 \\
5 \\
4\end{array}$ & $\begin{array}{l}214 \\
212 \\
215 \\
228 \\
222\end{array}$ & $\begin{array}{l}213 \\
213 \\
215 \\
215 \\
215\end{array}$ & +8 & II \\
\hline $\begin{array}{r}92.884 \\
98.166 \\
5899.007 \\
5901.462 \\
05.681\end{array}$ & $\begin{array}{l}\mathrm{B} \\
\mathrm{B} \\
\mathrm{C} \\
\mathrm{B} \\
\mathrm{A}\end{array}$ & $\begin{array}{r}19 \\
8 \\
2 \\
12 \\
17\end{array}$ & $\begin{array}{l}3.097 \mathrm{~s} \\
.378 \mathrm{~s} \\
.215 \\
.682 \mathrm{~s} \\
.895 \mathrm{~s}\end{array}$ & $\begin{array}{l}\mathrm{Ni} \\
\text { Air } \\
\text { Air } \\
\text { Air } \\
\text { Fe }\end{array}$ & $\begin{array}{l}4 \\
4 \\
2 \\
6 \\
4\end{array}$ & $\begin{array}{l}213 \\
212 \\
208 \\
220 \\
214\end{array}$ & $\begin{array}{l}215 \\
216 \\
216 \\
216 \\
216\end{array}$ & +6 & II \\
\hline $\begin{array}{l}08.999 \\
16.257 \\
18.441 \\
19.052 \\
19.645\end{array}$ & $\begin{array}{l}\mathrm{C} \\
\mathrm{B} \\
\mathrm{D} \\
\mathrm{C} \\
\mathrm{B}\end{array}$ & $\begin{array}{r}2 \\
17 \\
4 \\
8 \\
13\end{array}$ & $\begin{array}{l}9.213 \\
.475 \mathrm{~s} \\
.635 \\
.276 \\
.860 \mathrm{~s}\end{array}$ & $\begin{array}{l}\text { Air } \\
\text { Fe } \\
\text { Air } \\
\text { Air } \\
\text { Air }\end{array}$ & $\begin{array}{l}3 \\
3 \\
4 \\
5 \\
7\end{array}$ & $\begin{array}{l}214 \\
218 \\
194 \\
224 \\
215\end{array}$ & $\begin{array}{l}216 \\
216 \\
216 \\
216 \\
216\end{array}$ & & \\
\hline $\begin{array}{l}24.268 \\
27.797 \\
29.689 \\
30.192 \\
32.092\end{array}$ & $\begin{array}{l}\mathrm{D} \\
\mathrm{B} \\
\mathrm{C} \\
\mathrm{A} \\
\mathrm{C}\end{array}$ & $\begin{array}{r}5 \\
15 \\
12 \\
16 \\
10\end{array}$ & $\begin{array}{c}.490 \\
8.013 \\
.898 \\
.406 \mathrm{~s} \\
.306\end{array}$ & $\begin{array}{l}\text { Air } \\
\mathrm{Fe} \\
\mathrm{Fe} \\
\mathrm{Fe} \\
\mathrm{Air}\end{array}$ & $\begin{array}{l}4 \\
2 \\
2 \\
6 \\
5\end{array}$ & $\begin{array}{l}222 \\
216 \\
209 \\
214 \\
214\end{array}$ & $\begin{array}{l}216 \\
216 \\
216 \\
216 \\
216\end{array}$ & +11 & V \\
\hline $\begin{array}{l}34.665 \\
41.074 \\
42.568 \\
46.005 \\
47.057\end{array}$ & $\begin{array}{l}\mathrm{A} \\
\mathrm{C} \\
\mathrm{C} \\
\mathrm{C} \\
\mathrm{D}\end{array}$ & $\begin{array}{r}18 \\
4 \\
3 \\
11 \\
2\end{array}$ & $\begin{array}{l}.881 \mathrm{~s} \\
.290 \\
.789 \\
.223 \\
.283\end{array}$ & $\begin{array}{l}\mathrm{Fe} \\
\text { Air } \\
\text { Air } \\
\text { Air } \\
\text { Air }\end{array}$ & $\begin{array}{l}5 \\
5 \\
3 \\
3 \\
2\end{array}$ & $\begin{array}{l}216 \\
216 \\
221 \\
218 \\
226\end{array}$ & $\begin{array}{l}216 \\
217 \\
217 \\
217 \\
217\end{array}$ & +7 & V \\
\hline $\begin{array}{l}48.544 \\
52.726 \\
53.170 \\
56.706 \\
75.357\end{array}$ & $\begin{array}{l}\mathrm{B} \\
\mathrm{A} \\
\mathrm{C} \\
\mathrm{B} \\
\mathrm{C}\end{array}$ & $\begin{array}{r}14 \\
18 \\
6 \\
17 \\
9\end{array}$ & $\begin{array}{l}.765 \mathrm{~s} \\
.943 \\
.386 \\
.923 \mathrm{~s} \\
.575 \mathrm{~s}\end{array}$ & $\begin{array}{l}\mathrm{Si} \\
\mathrm{Fe} \\
\mathrm{Ti} \\
\mathrm{Fe} \\
\mathrm{Fe}\end{array}$ & $\begin{array}{l}6 \\
4 \\
1 \\
4 \\
3\end{array}$ & $\begin{array}{l}221 \\
217 \\
216 \\
217 \\
218\end{array}$ & $\begin{array}{l}217 \\
217 \\
217 \\
217 \\
218\end{array}$ & +8 & $\begin{array}{ll}\mathrm{V} & \\
\text { II } & \\
\mathrm{V} & \mathrm{b} \\
\mathrm{V} 4\end{array}$ \\
\hline $\begin{array}{l}76.788 \\
78.564 \\
83.689 \\
84.826 \\
87.067\end{array}$ & $\begin{array}{l}\mathrm{B} \\
\mathrm{C} \\
\mathrm{B} \\
\mathrm{B} \\
\mathrm{A}\end{array}$ & $\begin{array}{r}17 \\
3 \\
18 \\
19 \\
18\end{array}$ & $\begin{array}{c}7.007 \mathrm{~s} \\
.768 \\
.908 \\
5.040 \mathrm{~s} \\
.200 \mathrm{~s}\end{array}$ & $\begin{array}{l}\mathrm{Fe} \\
\mathrm{Ti} \\
\mathrm{Fe} \\
\mathrm{Fe} \\
\mathrm{Fe}\end{array}$ & $\begin{array}{l}4 \\
1 \\
5 \\
6 \\
5\end{array}$ & $\begin{array}{l}219 \\
204 \\
219 \\
214 \\
223\end{array}$ & $\begin{array}{l}218 \\
218 \\
218 \\
218 \\
218\end{array}$ & $\begin{array}{l}+9 \\
+5 \\
+9 \\
-3\end{array}$ & $\begin{array}{l}\text { V } \\
\text { II } \\
\text { V } \\
\text { IV } \\
\text { V }\end{array}$ \\
\hline $\begin{array}{r}91.382 \\
5997.786 \\
6003.023 \\
05.552 \\
07.321\end{array}$ & $\begin{array}{l}\mathrm{C} \\
\mathrm{C} \\
\mathrm{B} \\
\mathrm{D} \\
\mathrm{D}\end{array}$ & $\begin{array}{r}9 \\
11 \\
19 \\
6 \\
7\end{array}$ & $\begin{array}{l}.600 \\
8.002 \\
.239 \mathrm{~s} \\
.770 \\
.540\end{array}$ & $\begin{array}{l}\mathrm{Fe} \\
\mathrm{Fe} \\
\mathrm{Fe} \\
\mathrm{Fe}\end{array}$ & $\begin{array}{l}2 \\
2 \\
6 \\
1 \\
1\end{array}$ & $\begin{array}{l}218 \\
216 \\
216 \\
218 \\
219\end{array}$ & $\begin{array}{l}218 \\
217 \\
217 \\
217 \\
217\end{array}$ & +10 & $\mathrm{~V}$ \\
\hline $\begin{array}{l}07.965 \\
08.565 \\
13.497 \\
16.646 \\
21.800\end{array}$ & $\begin{array}{l}\mathrm{A} \\
\mathrm{A} \\
\mathrm{A} \\
\mathrm{B} \\
\mathrm{A}\end{array}$ & $\begin{array}{l}22 \\
21 \\
21 \\
21 \\
22\end{array}$ & $\begin{array}{r}8.186 \mathrm{~s} \\
.785 \mathrm{~s} \\
.715 \mathrm{~s} \\
.861 \mathrm{~s} \\
2.016 \mathrm{~s}\end{array}$ & $\begin{array}{l}\mathrm{Fe} \\
\mathrm{Fe} \\
\mathrm{Mn} \\
\mathrm{Mn} \\
\mathrm{Mn}\end{array}$ & $\begin{array}{l}4 \\
6 \\
6 \\
6 \\
6\end{array}$ & $\begin{array}{l}221 \\
220 \\
218 \\
215 \\
216\end{array}$ & $\begin{array}{l}217 \\
217 \\
216 \\
216 \\
216\end{array}$ & $\begin{array}{l}+6 \\
+7 \\
+7 \\
+7 \\
+3\end{array}$ & $\begin{array}{l}\text { V } \\
\text { III } \\
\text { III }\end{array}$ \\
\hline $\begin{array}{r}24.069 \\
27.058 \\
42.104 \\
56.005 \\
6065.495\end{array}$ & $\begin{array}{l}\mathrm{A} \\
\mathrm{A} \\
\mathrm{B} \\
\mathrm{B} \\
\mathrm{A}\end{array}$ & $\begin{array}{l}24 \\
26 \\
24 \\
27 \\
28\end{array}$ & $\begin{array}{l}.281 \mathrm{~s} \\
.274 \mathrm{~s} \\
.315 \mathrm{~s} \\
.227 \mathrm{~s} \\
.709 \mathrm{~s}\end{array}$ & $\begin{array}{l}\mathrm{Fe} \\
\mathrm{Fe} \\
\mathrm{Fe} \\
\mathrm{Fe} \\
\mathrm{Fe}\end{array}$ & $\begin{array}{r}7 \\
4 \\
3 \\
5 \\
5 \\
7\end{array}$ & $\begin{array}{l}212 \\
216 \\
211 \\
222 \\
214\end{array}$ & $\begin{array}{l}212 \\
215 \\
215 \\
214 \\
214\end{array}$ & $\begin{array}{r}+9 \\
+10 \\
+13 \\
+1 \\
+12\end{array}$ & $\begin{array}{ll}\text { V } & \\
\text { V } & \text { b4 } \\
\text { V } & \text { e } \\
\text { III } & \text { b4 }\end{array}$ \\
\hline
\end{tabular}


TABLE 1.-A. O. B. S. solar wave lengths-Continued

\begin{tabular}{|c|c|c|c|c|c|c|c|c|c|c|}
\hline \multirow{2}{*}{ A. O.B.S. A } & \multirow{2}{*}{$\begin{array}{l}\text { Proba- } \\
\text { ble } \\
\text { error }\end{array}$} & \multirow{2}{*}{$\begin{array}{l}\text { No. } \\
\text { obs. }\end{array}$} & \multicolumn{3}{|c|}{ Rowland } & \multicolumn{2}{|c|}{$\begin{array}{l}\text { Correction to } \\
\text { Rowland }\end{array}$} & \multirow{2}{*}{ Sun-are } & \multirow{2}{*}{\multicolumn{2}{|c|}{$\begin{array}{c}\text { Tempera- } \\
\text { ture and } \\
\text { pressure } \\
\text { classes }\end{array}$}} \\
\hline & & & $\lambda$ & Iden. & Int. & Obs. & Mean & & & \\
\hline $\begin{array}{r}6078.499 \\
79.016 \\
82.717 \\
84.121 \\
85.255\end{array}$ & $\begin{array}{l}\text { A } \\
\text { A } \\
\text { C } \\
\text { D } \\
\text { C }\end{array}$ & $\begin{array}{r}26 \\
23 \\
17 \\
3 \\
20\end{array}$ & $\begin{array}{l}.710 \mathrm{~s} \\
.227 \mathrm{~s} \\
.930 \\
.325 \\
.470\end{array}$ & $\begin{array}{l}\mathrm{Fe} \\
\mathrm{Fe} \\
\mathrm{Fe} \\
\mathrm{Fe}-\mathrm{Ti}\end{array}$ & $\begin{array}{l}5 \\
2 \\
1 \\
0 \\
2\end{array}$ & $\begin{array}{l}211 \\
211 \\
213 \\
204 \\
215\end{array}$ & $\begin{array}{l}213 \\
213 \\
213 \\
213 \\
213\end{array}$ & +3 & V & \\
\hline $\begin{array}{r}86.287 \\
89.572 \\
90.215 \\
93.649 \\
6096.673\end{array}$ & $\begin{array}{l}\text { B } \\
\text { C } \\
\text { C } \\
\text { B } \\
\text { B }\end{array}$ & $\begin{array}{l}17 \\
18 \\
12 \\
12 \\
17\end{array}$ & $\begin{array}{l}.500 \\
.787 \\
.429 \\
.864 \\
.880\end{array}$ & $\begin{array}{l}\mathrm{Ni} \\
\mathrm{Fe} \\
\mathrm{Fe} \\
\mathrm{Fe} \\
\mathrm{Fe}\end{array}$ & $\begin{array}{l}1 \\
1 \\
2 \\
2 \\
3\end{array}$ & $\begin{array}{l}213 \\
215 \\
214 \\
215 \\
207\end{array}$ & $\begin{array}{l}212 \\
212 \\
212 \\
212 \\
212\end{array}$ & & V & \\
\hline $\begin{array}{r}6102.182 \\
02.726 \\
03.207 \\
08.120 \\
11.078\end{array}$ & $\begin{array}{l}\mathrm{A} \\
\mathrm{A} \\
\mathrm{C} \\
\mathrm{A} \\
\mathrm{C}\end{array}$ & $\begin{array}{l}26 \\
22 \\
12 \\
26 \\
15\end{array}$ & $\begin{array}{l}.392 \mathrm{~s} \\
.937 \mathrm{~s} \\
.400 \mathrm{~s} \\
.334 \mathrm{~s} \\
.290 \mathrm{~s}\end{array}$ & $\begin{array}{l}\mathrm{Fe} \\
\mathrm{Ca} \\
\mathrm{Fe} \\
\mathrm{Ni} \\
\mathrm{Ni}\end{array}$ & $\begin{array}{l}6 \\
9 \\
4 \\
6 \\
2\end{array}$ & $\begin{array}{l}210 \\
211 \\
193 \\
214 \\
212\end{array}$ & $\begin{array}{l}212 \\
212 \\
212 \\
211 \\
211\end{array}$ & $\begin{array}{l}+7 \\
+6 \\
+13 \\
-1\end{array}$ & $\begin{array}{l}\text { V } \\
\text { II } \\
\text { V } \\
\text { II }\end{array}$ & \\
\hline $\begin{array}{l}16.197 \\
22.226 \\
25.021 \\
26.228 \\
27.912\end{array}$ & $\begin{array}{l}\mathrm{B} \\
\mathrm{B} \\
\mathrm{C} \\
\mathrm{B} \\
\mathrm{B}\end{array}$ & $\begin{array}{r}17 \\
21 \\
8 \\
8 \\
22\end{array}$ & $\begin{array}{l}.397 \mathrm{~s} \\
.434 \mathrm{~s} \\
.236 \\
.435 \\
8.124\end{array}$ & $\begin{array}{l}\mathrm{Ni} \\
\mathrm{Ca} \\
\mathrm{Ti} \\
\mathrm{Fe}\end{array}$ & $\begin{array}{r}4 \\
10 \\
1 \\
1 \\
3\end{array}$ & $\begin{array}{l}200 \\
208 \\
215 \\
207 \\
212\end{array}$ & $\begin{array}{l}211 \\
211 \\
211 \\
210 \\
210\end{array}$ & $\begin{array}{l}+10 \\
+11\end{array}$ & $\begin{array}{l}\text { V } \\
\text { II } \\
\text { II }\end{array}$ & b \\
\hline $\begin{array}{l}28.983 \\
36.621 \\
37.000 \\
37.701 \\
41.729\end{array}$ & $\begin{array}{l}\mathrm{C} \\
\mathrm{B} \\
\mathrm{A} \\
\mathrm{A} \\
\mathrm{A}\end{array}$ & $\begin{array}{r}9 \\
21 \\
24 \\
21 \\
24\end{array}$ & $\begin{array}{l}9.190 \\
.829 \mathrm{~s} \\
7.210 \\
.915 \\
.938 \mathrm{~s}\end{array}$ & $\begin{array}{c}\mathrm{Ni} \\
\mathrm{Fe} \\
\mathrm{Fe} \\
\mathrm{Fe} \\
\mathrm{Fe}-\mathrm{Ba}\end{array}$ & $\begin{array}{l}1 \\
8 \\
3 \\
7 \\
7\end{array}$ & $\begin{array}{l}207 \\
208 \\
210 \\
214 \\
209\end{array}$ & $\begin{array}{l}210 \\
210 \\
210 \\
210 \\
210\end{array}$ & $\begin{array}{l}+4 \\
+7\end{array}$ & $\begin{array}{l}\text { III } \\
\text { III } \\
\text { V }\end{array}$ & $\begin{array}{l}\mathrm{b} 4 \\
\mathrm{~b} \\
\mathrm{~b} 4\end{array}$ \\
\hline $\begin{array}{l}45.021 \\
49.248 \\
51.623 \\
54.229 \\
55.142\end{array}$ & $\begin{array}{l}\mathrm{B} \\
\mathrm{B} \\
\mathrm{A} \\
\mathrm{A} \\
\mathrm{B}\end{array}$ & $\begin{array}{l}14 \\
17 \\
24 \\
12 \\
16\end{array}$ & $\begin{array}{l}.228 \\
.458 \\
.834 \\
.438 \mathrm{~s} \\
.350\end{array}$ & $\begin{array}{l}\mathrm{Fe} \\
\mathrm{Na}\end{array}$ & $\begin{array}{l}2 \\
2 \\
4 \\
2 \\
7\end{array}$ & $\begin{array}{l}207 \\
210 \\
211 \\
209 \\
208\end{array}$ & $\begin{array}{l}210 \\
210 \\
210 \\
210 \\
210\end{array}$ & & & \\
\hline $\begin{array}{l}57.733 \\
60.750 \\
61.294 \\
62.181 \\
63.759\end{array}$ & $\begin{array}{l}\text { B } \\
\text { A } \\
\text { B } \\
\text { C } \\
\text { C }\end{array}$ & $\begin{array}{r}25 \\
23 \\
21 \\
16 \\
4\end{array}$ & $\begin{array}{l}.945 \\
.956 \mathrm{~s} \\
.503 \\
.390 \mathrm{~s} \\
.968\end{array}$ & $\begin{array}{l}\mathrm{Fe} \\
\mathrm{Na} \\
\mathrm{Ca} \\
\mathrm{Ca} \\
\mathrm{Ca}\end{array}$ & $\begin{array}{r}5 \\
3 \\
4 \\
15 \\
3\end{array}$ & $\begin{array}{l}212 \\
206 \\
209 \\
209 \\
209\end{array}$ & $\begin{array}{l}210 \\
210 \\
210 \\
210 \\
210\end{array}$ & +1 & $\begin{array}{l}\text { V } \\
\text { III } \\
\text { II } \\
\text { III }\end{array}$ & b4 \\
\hline $\begin{array}{l}65.363 \\
66.440 \\
69.040 \\
69.563 \\
70.517\end{array}$ & $\begin{array}{l}\text { B } \\
\text { A } \\
\text { A } \\
\text { A } \\
\text { B }\end{array}$ & $\begin{array}{l}24 \\
25 \\
25 \\
23 \\
21\end{array}$ & $\begin{array}{l}.577 \\
.651 \\
.249 \mathrm{~s} \\
.778 \mathrm{~s} \\
.730\end{array}$ & $\begin{array}{c}\mathrm{Fe} \\
\mathrm{Ca} \\
\mathrm{Ca} \\
\mathrm{Ca} \\
\mathrm{Fe}-\mathrm{Ni}\end{array}$ & $\begin{array}{l}3 \\
5 \\
6 \\
7 \\
6\end{array}$ & $\begin{array}{l}214 \\
211 \\
209 \\
215 \\
213\end{array}$ & $\begin{array}{l}210 \\
211 \\
211 \\
211 \\
211\end{array}$ & $\begin{array}{l}+9 \\
+7\end{array}$ & $\begin{array}{l}\text { III } \\
\text { III } \\
\text { III } \\
\text { V }\end{array}$ & $\mathrm{b}$ \\
\hline $\begin{array}{r}73.339 \\
6175.370 \\
76.816 \\
80.208 \\
86.717\end{array}$ & $\begin{array}{l}\mathrm{A} \\
\mathrm{A} \\
\mathrm{A} \\
\mathrm{A} \\
\mathrm{C}\end{array}$ & $\begin{array}{l}27 \\
23 \\
27 \\
26 \\
13\end{array}$ & $\begin{array}{c}.553 \mathrm{~s} \\
.584 \\
7.027 \mathrm{~s} \\
.420 \mathrm{~s} \\
.928\end{array}$ & $\begin{array}{l}\mathrm{Fe} \\
\mathrm{Ni} \\
\mathrm{Ni} \\
\mathrm{Fe} \\
\mathrm{Ni}\end{array}$ & $\begin{array}{l}5 \\
3 \\
5 \\
5 \\
2\end{array}$ & $\begin{array}{l}214 \\
214 \\
211 \\
212 \\
211\end{array}$ & $\begin{array}{l}211 \\
211 \\
212 \\
212 \\
212\end{array}$ & +3 & $\begin{array}{l}\mathrm{III}_{\mathrm{V}} \\
\mathrm{V}\end{array}$ & $\mathrm{b} 4$ \\
\hline $\begin{array}{r}87.994 \\
91.181 \\
6191.570 \\
6200.320 \\
04.619\end{array}$ & $\begin{array}{l}\mathrm{A} \\
\mathrm{A} \\
\mathrm{A} \\
\mathrm{A} \\
\mathrm{D}\end{array}$ & $\begin{array}{r}18 \\
26 \\
20 \\
25 \\
3\end{array}$ & $\begin{array}{l}8.210 \\
.393 \mathrm{~s} \\
.779 \mathrm{~s} \\
.527 \mathrm{~s} \\
.825\end{array}$ & $\begin{array}{l}\mathrm{Fe} \\
\mathrm{Ni} \\
\mathrm{Fe} \\
\mathrm{Fe} \\
\mathrm{Ni}\end{array}$ & $\begin{array}{l}4 \\
6 \\
9 \\
6 \\
1\end{array}$ & $\begin{array}{l}216 \\
212 \\
209 \\
207 \\
206\end{array}$ & $\begin{array}{l}212 \\
211 \\
211 \\
210 \\
210\end{array}$ & +11 & $\begin{array}{l}\text { I } \\
\text { II } \\
\text { IV } \\
\text { III }\end{array}$ & $\begin{array}{l}\mathrm{b} 4 \\
\mathrm{~b} 4\end{array}$ \\
\hline $\begin{array}{l}13.436 \\
15.147 \\
16.359 \\
19.286 \\
23.986\end{array}$ & $\begin{array}{l}\text { B } \\
\text { A } \\
\text { B } \\
\text { A } \\
\text { C }\end{array}$ & $\begin{array}{l}26 \\
16 \\
12 \\
28 \\
10\end{array}$ & $\begin{array}{l}.644 \mathrm{~s} \\
.360 \\
.567 \\
.494 \mathrm{~s} \\
4.198\end{array}$ & $\begin{array}{l}\mathrm{Fe} \\
\mathrm{Fe} \\
\mathrm{V} ? \\
\mathrm{Fe} \\
\mathrm{Ni}\end{array}$ & $\begin{array}{l}6 \\
5 \\
1 \\
6 \\
1\end{array}$ & $\begin{array}{l}208 \\
213 \\
208 \\
208 \\
212\end{array}$ & $\begin{array}{l}209 \\
209 \\
209 \\
209 \\
209\end{array}$ & \pm 0 & $\begin{array}{l}\text { III } \\
\text { III }\end{array}$ & $\begin{array}{l}\mathrm{b} 4 \\
\mathrm{~b} 4 \\
\mathrm{~b} 4\end{array}$ \\
\hline $\begin{array}{l}26.740 \\
29.229 \\
30.736 \\
32.645 \\
37.322\end{array}$ & $\begin{array}{l}\mathrm{B} \\
\mathrm{B} \\
\mathrm{B} \\
\mathrm{A} \\
\mathrm{C}\end{array}$ & $\begin{array}{l}12 \\
19 \\
25 \\
27 \\
16\end{array}$ & $\begin{array}{l}.951 \\
.437 \\
.943 \mathrm{~s} \\
.856 \\
.534 \mathrm{~s}\end{array}$ & $\begin{array}{c}\mathrm{Fe} \\
\mathrm{Fe} \\
\mathrm{V}-\mathrm{Fe} \\
\mathrm{Fe}\end{array}$ & $\begin{array}{l}1 \\
1 \\
8 \\
3 \\
3\end{array}$ & $\begin{array}{l}211 \\
208 \\
207 \\
211 \\
212\end{array}$ & $\begin{array}{l}209 \\
210 \\
210 \\
210 \\
210\end{array}$ & +1. & $\begin{array}{l}\text { III } \\
\text { V }\end{array}$ & b4 \\
\hline $\begin{array}{r}38.387 \\
40.652 \\
43.114 \\
43.813 \\
6244.476\end{array}$ & $\begin{array}{l}\mathrm{B} \\
\mathrm{B} \\
\mathrm{D} \\
\mathrm{C} \\
\mathrm{C}\end{array}$ & $\begin{array}{r}14 \\
20 \\
6 \\
12 \\
12\end{array}$ & $\begin{array}{r}.598 \\
.863 \\
.320 \\
4.033 \\
.686\end{array}$ & $\underset{\mathrm{V}}{\mathrm{Fe}}$ & $\begin{array}{l}2 \\
3 \\
1 \\
2 \\
2\end{array}$ & $\begin{array}{l}211 \\
211 \\
216 \\
213 \\
210\end{array}$ & $\begin{array}{l}210 \\
210 \\
210 \\
210 \\
210\end{array}$ & & & \\
\hline
\end{tabular}


Table 1.-A. O. B. S. solar wave lengths-Continued

\begin{tabular}{|c|c|c|c|c|c|c|c|c|c|c|}
\hline \multirow{2}{*}{ A. O.B.S.A } & \multirow{2}{*}{$\begin{array}{l}\text { Proba- } \\
\text { ble } \\
\text { error }\end{array}$} & \multirow{2}{*}{$\begin{array}{l}\text { No. } \\
\text { obs. }\end{array}$} & \multicolumn{3}{|c|}{ Rowland } & \multicolumn{2}{|c|}{$\begin{array}{l}\text { Correction to } \\
\text { Rowland }\end{array}$} & \multirow{2}{*}{ Sun-are } & \multirow{2}{*}{\multicolumn{2}{|c|}{$\begin{array}{l}\text { Tempera- } \\
\text { ture and } \\
\text { pressure } \\
\text { classes }\end{array}$}} \\
\hline & & & $\lambda$ & Iden. & Int. & Obs. & Mean & & & \\
\hline $\begin{array}{r}6245.621 \\
46.328 \\
47.558 \\
52.562 \\
54.254\end{array}$ & $\begin{array}{l}\text { A } \\
\text { B } \\
\text { B } \\
\text { A } \\
\text { B }\end{array}$ & $\begin{array}{l}11 \\
22 \\
20 \\
22 \\
20\end{array}$ & $\begin{array}{l}.832 \\
.535 \mathrm{~s} \\
.774 \\
.773 \mathrm{~s} \\
.456 \mathrm{~s}\end{array}$ & $\begin{array}{l}\mathrm{Fe} \\
\mathrm{V} \\
\mathrm{Fe} \\
\mathrm{Fe}\end{array}$ & $\begin{array}{l}1 \\
8 \\
2 \\
7 \\
5\end{array}$ & $\begin{array}{l}211 \\
207 \\
216 \\
211 \\
202\end{array}$ & $\begin{array}{l}210 \\
210 \\
210 \\
210 \\
210\end{array}$ & $\begin{array}{l}+10 \\
+6\end{array}$ & $\begin{array}{l}\text { V } \\
\text { III } \\
\text { III }\end{array}$ & $\begin{array}{l}\mathrm{b4} \\
\mathrm{b4}\end{array}$ \\
\hline $\begin{array}{l}56.366 \\
58.110 \\
58.712 \\
61.103 \\
65.138\end{array}$ & $\begin{array}{l}\text { A } \\
\text { B } \\
\text { B } \\
\text { A } \\
\text { A }\end{array}$ & $\begin{array}{l}22 \\
20 \\
19 \\
19 \\
19\end{array}$ & $\begin{array}{l}.572 \mathrm{~s} \\
.322 \\
.927 \\
.316 \mathrm{~s} \\
.348 \mathrm{~s}\end{array}$ & $\begin{array}{c}\mathrm{Ni}-\mathrm{Fe} \\
\mathrm{Ti} \\
\mathrm{Ti} \\
\mathrm{Ti} \\
\mathrm{Fe}\end{array}$ & $\begin{array}{l}6 \\
2 \\
3 \\
1 \\
5\end{array}$ & $\begin{array}{l}206 \\
212 \\
215 \\
213 \\
210\end{array}$ & $\begin{array}{l}210 \\
210 \\
210 \\
210 \\
209\end{array}$ & $\begin{array}{l}+4 \\
+6 \\
+2 \\
+8\end{array}$ & $\begin{array}{l}\text { III } \\
\text { II } \\
\text { II } \\
\text { II }\end{array}$ & $\begin{array}{l}\mathrm{b} \\
\mathrm{b} 4\end{array}$ \\
\hline $\begin{array}{l}\text { 70. } 231 \\
71.280 \\
78.093 \\
78.881 \\
79.102\end{array}$ & $\begin{array}{l}\text { A } \\
\text { C } \\
\text { B } \\
\text { A } \\
\text { A }\end{array}$ & $\begin{array}{r}20 \\
7 \\
23 \\
8 \\
9\end{array}$ & $\begin{array}{r}.442 \\
.486 \\
.303 \\
9.084 \\
.308\end{array}$ & $\begin{array}{l}\text { Fe } \\
\text { Fe } \\
\text { Air } \\
\text { Air } \\
\text { Air }\end{array}$ & $\begin{array}{l}3 \\
0 \\
4 \\
2 \\
3\end{array}$ & $\begin{array}{l}211 \\
206 \\
210 \\
203 \\
206\end{array}$ & $\begin{array}{l}209 \\
209 \\
208 \\
208 \\
208\end{array}$ & 4 & III & $\mathrm{b}$ \\
\hline $\begin{array}{l}79.897 \\
80.393 \\
80.623 \\
81.178 \\
81.955\end{array}$ & $\begin{array}{l}\text { A } \\
\text { A } \\
\text { C } \\
\text { A } \\
\text { A }\end{array}$ & $\begin{array}{r}2 \\
9 \\
6 \\
14 \\
15\end{array}$ & $\begin{array}{r}0.108 \\
.598 \\
.833 \\
.387 \\
2.164\end{array}$ & $\begin{array}{l}\text { Air } \\
\text { Air } \\
\text { Fe } \\
\text { Air } \\
\text { Air }\end{array}$ & $\begin{array}{l}2 \\
2 \\
3 \\
1 \\
2\end{array}$ & $\begin{array}{l}211 \\
205 \\
210 \\
209 \\
209\end{array}$ & $\begin{array}{l}208 \\
208 \\
208 \\
208 \\
208\end{array}$ & & IA & $\mathbf{8}$ \\
\hline $\begin{array}{l}82.730 \\
83.797 \\
87.750 \\
89.398 \\
90.220\end{array}$ & $\begin{array}{l}\text { A } \\
\text { A } \\
\text { A } \\
\text { C } \\
\text { A }\end{array}$ & $\begin{array}{r}3 \\
8 \\
3 \\
10 \\
26\end{array}$ & $\begin{array}{r}.933 \\
4.002 \\
.953 \\
.606 \\
.427\end{array}$ & $\begin{array}{l}\text { Air } \\
\text { Air } \\
\text { Air } \\
\text { Air } \\
\text { Air }\end{array}$ & $\begin{array}{l}2 \\
1 \\
1 \\
1 \\
2\end{array}$ & $\begin{array}{l}203 \\
205 \\
203 \\
208 \\
207\end{array}$ & $\begin{array}{l}208 \\
208 \\
208 \\
208 \\
208\end{array}$ & & & \\
\hline $\begin{array}{l}90.967 \\
92.161 \\
92.959 \\
95.178 \\
95.959\end{array}$ & $\begin{array}{l}\text { C } \\
\text { A } \\
\mathbf{A} \\
\mathbf{A} \\
\mathbf{A}\end{array}$ & $\begin{array}{l}13 \\
26 \\
26 \\
23 \\
26\end{array}$ & $\begin{array}{r}1.184 \\
.373 \\
3.170 \\
.389 \\
6.170\end{array}$ & $\begin{array}{l}\text { Fe } \\
\text { Air } \\
\text { Air } \\
\text { Air } \\
\text { Air }\end{array}$ & $\begin{array}{l}4 \\
2 \\
3 \\
3 \\
3\end{array}$ & $\begin{array}{l}217 \\
212 \\
211 \\
211 \\
211\end{array}$ & $\begin{array}{l}208 \\
208 \\
208 \\
209 \\
209\end{array}$ & (1) & & e? \\
\hline $\begin{array}{r}97.797 \\
6298.453 \\
6299.227 \\
6301.505 \\
01.998\end{array}$ & $\begin{array}{l}\text { A } \\
\text { A } \\
\text { A } \\
\text { A } \\
\text { A }\end{array}$ & $\begin{array}{l}19 \\
26 \\
26 \\
18 \\
21\end{array}$ & $\begin{array}{r}8.007 \\
.666 \\
.436 \\
.718 \\
.209\end{array}$ & $\begin{array}{l}\mathrm{Fe} \\
\mathrm{Air} \\
\mathrm{Air} \\
\mathrm{Fe} \\
\mathrm{Air}\end{array}$ & $\begin{array}{l}5 \\
2 \\
3 \\
7 \\
2\end{array}$ & $\begin{array}{l}210 \\
213 \\
209 \\
213 \\
211\end{array}$ & $\begin{array}{l}209 \\
210 \\
210 \\
211 \\
211\end{array}$ & $\begin{array}{l}+7 \\
+4\end{array}$ & $\begin{array}{l}\text { III } \\
\text { IV }\end{array}$ & $\mathrm{b} 4$ \\
\hline $\begin{array}{l}02.497 \\
02.764 \\
05.810 \\
06.564 \\
09.887\end{array}$ & $\begin{array}{l}\text { B } \\
\text { A } \\
\text { A } \\
\text { A } \\
\text { A }\end{array}$ & $\begin{array}{l}14 \\
16 \\
20 \\
20 \\
18\end{array}$ & $\begin{array}{r}.709 \\
.975 \\
6.024 \\
.780 \\
0.101\end{array}$ & $\begin{array}{l}\text { Fe } \\
\text { Air } \\
\text { Air } \\
\text { Air } \\
\text { Air }\end{array}$ & $\begin{array}{l}5 \\
2 \\
2 \\
2 \\
2\end{array}$ & $\begin{array}{l}212 \\
211 \\
214 \\
216 \\
214\end{array}$ & $\begin{array}{l}211 \\
211 \\
212 \\
212 \\
213\end{array}$ & +4 & V & \\
\hline $\begin{array}{l}10.663 \\
14.663 \\
15.314 \\
15.814 \\
18.025\end{array}$ & $\begin{array}{l}\mathrm{A} \\
\mathrm{B} \\
\mathrm{B} \\
\mathbf{C} \\
\mathrm{B}\end{array}$ & $\begin{array}{r}12 \\
16 \\
13 \\
8 \\
17\end{array}$ & $\begin{array}{r}.848 \\
.876 \\
.517 \\
6.028 \\
.239\end{array}$ & $\begin{array}{l}\mathrm{Air} \\
\mathrm{Ni} \\
\mathrm{Fe} \\
\mathrm{Fe} \\
\mathrm{Fe}\end{array}$ & $\begin{array}{l}1 \\
4 \\
2 \\
1 \\
6\end{array}$ & $\begin{array}{l}215 \\
213 \\
203 \\
214 \\
214\end{array}$ & $\begin{array}{l}213 \\
213 \\
213 \\
213 \\
214\end{array}$ & +7 & $\begin{array}{l}\text { II } \\
\text { III }\end{array}$ & $\begin{array}{l}\mathrm{b} \\
\mathrm{b} 4\end{array}$ \\
\hline $\begin{array}{l}18.710 \\
22.693 \\
27.604 \\
30.098 \\
30.852\end{array}$ & $\begin{array}{l}\mathbf{A} \\
\mathbf{B} \\
\mathbf{C} \\
\mathbf{B} \\
\mathbf{C}\end{array}$ & $\begin{array}{r}4 \\
19 \\
13 \\
12 \\
11\end{array}$ & $\begin{array}{r}.919 \\
.907 \\
.820 \\
.316 \\
1.067\end{array}$ & $\begin{array}{l}\mathrm{Fe} \\
\mathrm{Ni} \\
\mathrm{Cr} \\
\mathrm{Fe}\end{array}$ & $\begin{array}{l}1 \\
4 \\
2 \\
1 \\
2\end{array}$ & $\begin{array}{l}209 \\
214 \\
216 \\
218 \\
215\end{array}$ & $\begin{array}{l}214 \\
214 \\
215 \\
215 \\
215\end{array}$ & +5 & $\begin{array}{l}\text { III } \\
\text { II } \\
\text { IA }\end{array}$ & b \\
\hline $\begin{array}{l}35.337 \\
36.830 \\
38.874 \\
39.115 \\
44.157\end{array}$ & $\begin{array}{l}\text { A } \\
\text { A } \\
\text { B } \\
\text { D } \\
\text { A }\end{array}$ & $\begin{array}{r}20 \\
20 \\
5 \\
5 \\
19\end{array}$ & $\begin{array}{r}.554 \\
7.048 \\
9.096 \\
.335 \\
.371\end{array}$ & $\begin{array}{l}\mathrm{Fe} \\
\mathrm{Fe} \\
\mathrm{Fe} \\
\mathrm{Fe} \\
\mathrm{Fe}\end{array}$ & $\begin{array}{l}6 \\
7 \\
2 \\
2 \\
4\end{array}$ & $\begin{array}{l}217 \\
218 \\
212 \\
220 \\
214\end{array}$ & $\begin{array}{l}215 \\
215 \\
215 \\
215 \\
215\end{array}$ & $\begin{array}{l}+12 \\
+6\end{array}$ & $\begin{array}{l}\underset{V}{\text { III }} \\
\text { III }\end{array}$ & b \\
\hline $\begin{array}{l}47.087 \\
55.036 \\
58.685 \\
62.869 \\
78.256\end{array}$ & $\begin{array}{l}\mathrm{D} \\
\mathrm{B} \\
\mathrm{B} \\
\mathrm{B} \\
\mathrm{C}\end{array}$ & $\begin{array}{r}10 \\
20 \\
18 \\
7 \\
11\end{array}$ & $\begin{array}{r}.310 \\
.246 \\
.898 \\
3.090 \\
.468\end{array}$ & $\begin{array}{c}\mathrm{Fe} \\
\mathrm{Fe} \\
\mathrm{Cr}-\mathrm{Fe} \\
\mathrm{Ni}\end{array}$ & $\begin{array}{l}2 \\
4 \\
6 \\
2 \\
2\end{array}$ & $\begin{array}{l}223 \\
210 \\
213 \\
221 \\
212\end{array}$ & $\begin{array}{l}215 \\
215 \\
214 \\
214 \\
211\end{array}$ & +5 & $\begin{array}{l}\text { III } \\
\text { IA } \\
\text { IV }\end{array}$ & \\
\hline $\begin{array}{r}80.751 \\
6393.611 \\
6400.005 \\
00.326 \\
6408.026\end{array}$ & $\begin{array}{l}\mathrm{C} \\
\mathbf{B} \\
\mathbf{B} \\
\mathrm{C} \\
\mathrm{B}\end{array}$ & $\begin{array}{l}19 \\
19 \\
17 \\
13 \\
20\end{array}$ & $\begin{array}{l}.958 \\
.820 \mathrm{~s} \\
.217 \mathrm{~s} \\
.538 \mathrm{~s} \\
.233 \mathrm{~s}\end{array}$ & $\begin{array}{l}\mathrm{Fe} \\
\mathrm{Fe} \\
\mathrm{Fe} \\
\mathrm{Fe} \\
\mathrm{Fe}\end{array}$ & $\begin{array}{l}4 \\
7 \\
8 \\
2 \\
5\end{array}$ & $\begin{array}{l}207 \\
209 \\
212 \\
212 \\
207\end{array}$ & $\begin{array}{l}211 \\
211 \\
211 \\
211 \\
211\end{array}$ & $\begin{array}{l}+5 \\
+10 \\
+3 \\
+8\end{array}$ & $\begin{array}{l}\text { V } \\
\text { III } \\
\text { III } \\
\text { V }\end{array}$ & $\begin{array}{l}\mathrm{b} \\
\mathrm{b} 4\end{array}$ \\
\hline
\end{tabular}


TABLe 1.-A. O. B. S. solar wave lengths-Continued

\begin{tabular}{|c|c|c|c|c|c|c|c|c|c|}
\hline \multirow{2}{*}{ A. O.B.S. $\lambda$} & \multirow{2}{*}{$\begin{array}{l}\text { Proba- } \\
\text { ble } \\
\text { error }\end{array}$} & \multirow{2}{*}{$\begin{array}{l}\text { No. } \\
\text { obs. }\end{array}$} & \multicolumn{3}{|c|}{ Rowland } & \multicolumn{2}{|c|}{$\begin{array}{l}\text { Correction to } \\
\text { Rowland }\end{array}$} & \multirow{2}{*}{ Sun-arc } & \multirow{2}{*}{$\begin{array}{l}\text { Tempera- } \\
\text { ture and } \\
\text { pressure } \\
\text { classes }\end{array}$} \\
\hline & & & $\lambda$ & Iden. & Int. & Obs. & Mean & & \\
\hline $\begin{array}{r}6411.656 \\
14.995 \\
16.930 \\
19.954 \\
21.360\end{array}$ & $\begin{array}{l}\mathrm{B} \\
\mathrm{C} \\
\mathrm{C} \\
\mathrm{A} \\
\mathrm{B}\end{array}$ & $\begin{array}{r}20 \\
9 \\
11 \\
20 \\
21\end{array}$ & $\begin{array}{l}.865 \mathrm{~s} \\
5.199 \\
7.133 \\
0.169 \mathrm{~s} \\
.570 \mathrm{~s}\end{array}$ & $\begin{array}{l}\mathrm{Fe} \\
\mathrm{Fe} \\
\mathrm{Fe} \\
\mathrm{Fe}\end{array}$ & $\begin{array}{l}7 \\
1 \\
1 \\
4 \\
7\end{array}$ & $\begin{array}{l}209 \\
204 \\
203 \\
215 \\
210\end{array}$ & $\begin{array}{l}211 \\
211 \\
211 \\
211 \\
211\end{array}$ & $\begin{array}{l}+8 \\
+5 \\
+5 \\
+10\end{array}$ & $\begin{array}{l}\text { IV } \\
\text { V } \\
\text { III b }\end{array}$ \\
\hline $\begin{array}{l}30.854 \\
32.686 \\
39.085 \\
49.820 \\
55.605\end{array}$ & $\begin{array}{l}\mathrm{B} \\
\mathrm{B} \\
\mathrm{A} \\
\mathrm{B} \\
\mathrm{B}\end{array}$ & $\begin{array}{l}21 \\
13 \\
22 \\
23 \\
23\end{array}$ & $\begin{array}{c}1.066 \mathrm{~s} \\
.895 \\
.293 \mathrm{~s} \\
0.033 \mathrm{~s} \\
.820\end{array}$ & $\begin{array}{l}\mathrm{Fe} \\
\mathrm{Fe} \\
\mathrm{Ca} \\
\mathrm{Ca} \\
\mathrm{Ca}\end{array}$ & $\begin{array}{l}5 \\
1 \\
8 \\
6 \\
2\end{array}$ & $\begin{array}{l}212 \\
209 \\
208 \\
213 \\
215\end{array}$ & $\begin{array}{l}211 \\
211 \\
211 \\
212 \\
213\end{array}$ & $\begin{array}{l}+10 \\
+13 \\
+10 \\
+7\end{array}$ & $\begin{array}{l}\text { III b4 } \\
\text { II } \\
\text { II } \\
\text { II }\end{array}$ \\
\hline $\begin{array}{l}56.391 \\
62.559 \\
62.733 \\
69.189 \\
71.668\end{array}$ & $\begin{array}{l}\mathrm{B} \\
\mathrm{D} \\
\mathrm{D} \\
\mathrm{B} \\
\mathrm{A}\end{array}$ & $\begin{array}{r}22 \\
3 \\
3 \\
15 \\
23\end{array}$ & $\begin{array}{l}.603 \\
.784 \mathrm{~s} \\
.965 \\
.408 \\
.885 \mathrm{~s}\end{array}$ & $\begin{array}{l}\mathrm{Fe} \\
\mathrm{Ca} \\
\mathrm{Fe} \\
\mathrm{Fe} \\
\mathrm{Ca}\end{array}$ & $\begin{array}{l}3 \\
5 \\
3 \\
2 \\
5\end{array}$ & $\begin{array}{l}212 \\
225 \\
232 \\
219 \\
217\end{array}$ & $\begin{array}{l}213 \\
214 \\
214 \\
216 \\
216\end{array}$ & $\begin{array}{l}-6 \\
+8 \\
+8\end{array}$ & $\begin{array}{ll}\text { II } & \\
\text { V } & \text { b } \\
\text { II } & \end{array}$ \\
\hline $\begin{array}{l}75.632 \\
80.057 \\
81.875 \\
82.810 \\
83.239\end{array}$ & $\begin{array}{l}\text { D } \\
\text { A } \\
\text { B } \\
\text { B } \\
\text { A }\end{array}$ & $\begin{array}{r}7 \\
22 \\
25 \\
13 \\
8\end{array}$ & $\begin{array}{l}.846 \\
.285 \mathrm{~s} \\
2.098 \\
3.027 \\
.468\end{array}$ & $\begin{array}{l}\mathrm{Fe} \\
\mathrm{Air} \\
\mathrm{Fe} \\
\mathrm{Ni} \\
\text { Air }\end{array}$ & $\begin{array}{l}2 \\
1 \\
3 \\
1 \\
1\end{array}$ & $\begin{array}{l}214 \\
218 \\
223 \\
217 \\
229\end{array}$ & $\begin{array}{l}217 \\
218 \\
218 \\
219 \\
219\end{array}$ & 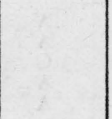 & $\begin{array}{l}\text { IV } \quad b \\
\text { IV } \\
\text { V }\end{array}$ \\
\hline $\begin{array}{r}86.777 \\
6490.790 \\
91.574 \\
92.903 \\
93.789\end{array}$ & $\begin{array}{l}\mathrm{C} \\
\mathrm{A} \\
\mathrm{C} \\
\mathrm{A} \\
\mathrm{A}\end{array}$ & $\begin{array}{r}3 \\
29 \\
13 \\
13 \\
31\end{array}$ & $\begin{array}{l}7.005 \\
1.015 \\
.800 \\
3.130 \\
4.004 \mathrm{~s}\end{array}$ & $\begin{array}{l}\text { Air } \\
\text { Air } \\
\text { Air } \\
\text { Air } \\
\text { Ca }\end{array}$ & $\begin{array}{l}0 \\
1 \\
1 \\
1 \\
6\end{array}$ & $\begin{array}{l}228 \\
225 \\
226 \\
227 \\
215\end{array}$ & $\begin{array}{l}219 \\
220 \\
220 \\
221 \\
221\end{array}$ & +9 & II \\
\hline $\begin{array}{r}94.995 \\
96.469 \\
96.911 \\
98.945 \\
6499.654\end{array}$ & $\begin{array}{l}\text { B } \\
\text { A } \\
\text { A } \\
\text { A } \\
\text { A }\end{array}$ & $\begin{array}{l}31 \\
22 \\
33 \\
28 \\
32\end{array}$ & $\begin{array}{c}.213 \mathrm{~s} \\
.688 \\
7.128 \\
9.168 \\
.880 \mathrm{~s}\end{array}$ & $\begin{array}{l}\mathrm{Fe} \\
\mathrm{Fe} \\
\mathrm{Fe} \\
\mathrm{Fe} \\
\mathrm{Ca}\end{array}$ & $\begin{array}{l}8 \\
2 \\
4 \\
1 \\
4\end{array}$ & $\begin{array}{l}218 \\
219 \\
217 \\
223 \\
226\end{array}$ & $\begin{array}{l}221 \\
222 \\
222 \\
222 \\
222\end{array}$ & $\begin{array}{l}+14 \\
+5 \\
+6\end{array}$ & $\begin{array}{l}\text { II b4 } \\
\text { II }\end{array}$ \\
\hline $\begin{array}{r}6504.193 \\
08.596 \\
14.730 \\
16.084 \\
18.374\end{array}$ & $\begin{array}{l}\text { D } \\
\text { D } \\
\text { A } \\
\text { A } \\
\text { B }\end{array}$ & $\begin{array}{r}3 \\
5 \\
28 \\
20 \\
20\end{array}$ & $\begin{array}{l}.415 \\
.826 \\
.956 \\
.311 \mathrm{~s} \\
.599 \mathrm{~s}\end{array}$ & $\begin{array}{l}\text { Air } \\
\text { Air } \\
\text { Air } \\
\text { Fe } \\
\text { Fe }\end{array}$ & $\begin{array}{l}0 \\
0 \\
2 \\
2 \\
2\end{array}$ & $\begin{array}{l}222 \\
230 \\
226 \\
227 \\
225\end{array}$ & $\begin{array}{l}223 \\
224 \\
226 \\
226 \\
227\end{array}$ & 2 & b \\
\hline $\begin{array}{l}23.839 \\
32.352 \\
33.946 \\
43.904 \\
46.247\end{array}$ & $\begin{array}{l}\mathrm{B} \\
\mathrm{C} \\
\mathrm{C} \\
\mathrm{A} \\
\mathrm{A}\end{array}$ & $\begin{array}{l}12 \\
13 \\
15 \\
26 \\
28\end{array}$ & $\begin{array}{l}4.080 \\
.595 \mathrm{~s} \\
4.172 \mathrm{~s} \\
4.140 \\
.479 \mathrm{~s}\end{array}$ & $\begin{array}{c}\text { Air } \\
\text { Air } \\
\text { Air } \\
\text { Air } \\
\text { Ti-Fe }\end{array}$ & $\begin{array}{l}1 \\
1 \\
2 \\
2 \\
6\end{array}$ & $\begin{array}{l}241 \\
243 \\
226 \\
236 \\
232\end{array}$ & $\begin{array}{l}229 \\
232 \\
232 \\
235 \\
235\end{array}$ & +8 & III $b$ \\
\hline $\begin{array}{l}47.692 \\
48.619 \\
52.626 \\
55.463 \\
64.197\end{array}$ & $\begin{array}{l}\text { B } \\
\text { B } \\
\text { B } \\
\text { C } \\
\text { C }\end{array}$ & $\begin{array}{r}10 \\
15 \\
20 \\
8 \\
5\end{array}$ & $\begin{array}{l}.945 \\
.855 \\
.865 \mathrm{~s} \\
.700 \\
.450\end{array}$ & $\begin{array}{l}\text { Air } \\
\text { Air } \\
\text { Air } \\
\text { Air }\end{array}$ & $\begin{array}{l}0 \\
1 \\
1 \\
1 \\
0\end{array}$ & $\begin{array}{l}253 \\
236 \\
239 \\
237 \\
253\end{array}$ & $\begin{array}{l}235 \\
236 \\
236 \\
236 \\
237\end{array}$ & 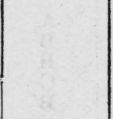 & \\
\hline $\begin{array}{l}\text { 69. } 223 \\
72.083 \\
72.786 \\
74.234 \\
75.022\end{array}$ & $\begin{array}{l}\mathrm{A} \\
\mathrm{C} \\
\mathrm{B} \\
\mathrm{C} \\
\mathrm{C}\end{array}$ & $\begin{array}{l}28 \\
11 \\
15 \\
13 \\
12\end{array}$ & $\begin{array}{l}.460 \mathrm{~s} \\
.330 \\
3.030 \\
.468 \mathrm{~s} \\
.270 \mathrm{~s}\end{array}$ & $\begin{array}{l}\mathrm{Fe} \\
\mathrm{Air} \\
\mathrm{Ca} \\
\mathrm{Fe} \\
\mathrm{Fe}\end{array}$ & $\begin{array}{l}5 \\
1 \\
1 \\
1 \\
2\end{array}$ & $\begin{array}{l}237 \\
247 \\
244 \\
234 \\
248\end{array}$ & $\begin{array}{l}238 \\
238 \\
238 \\
238 \\
238\end{array}$ & +9 & $\begin{array}{lll}\mathrm{V} & \\
\mathrm{IA} & \\
& \mathrm{b}\end{array}$ \\
\hline $\begin{array}{r}86.317 \\
92.926 \\
93.881 \\
6597.561 \\
6604.594\end{array}$ & $\begin{array}{l}\mathrm{C} \\
\mathrm{A} \\
\mathrm{A} \\
\mathrm{B} \\
\mathrm{B}\end{array}$ & $\begin{array}{l}13 \\
29 \\
29 \\
19 \\
17\end{array}$ & $\begin{array}{l}.550 \\
3.161 \mathrm{~s} \\
4.121 \mathrm{~s} \\
.807 \\
.837\end{array}$ & $\begin{array}{c}\mathrm{Ni} \\
\mathrm{Fe} \\
\mathrm{Fe} \\
\mathrm{Cr} ? \mathrm{Fe} \text { ? } \\
\mathrm{Fe}\end{array}$ & $\begin{array}{l}1 \\
6 \\
4 \\
1 \\
1\end{array}$ & $\begin{array}{l}233 \\
235 \\
240 \\
246 \\
243\end{array}$ & $\begin{array}{l}239 \\
240 \\
240 \\
240 \\
240\end{array}$ & $\begin{array}{l}+13 \\
+6\end{array}$ & $\begin{array}{l}\text { II } \\
\text { III } \quad b\end{array}$ \\
\hline $\begin{array}{l}09.118 \\
27.551 \\
33.753 \\
43.638 \\
63.451\end{array}$ & $\begin{array}{l}\mathrm{A} \\
\mathrm{C} \\
\mathrm{A} \\
\mathrm{A} \\
\mathrm{A}\end{array}$ & $\begin{array}{l}27 \\
12 \\
27 \\
31 \\
30\end{array}$ & $\begin{array}{l}.360 \mathrm{~s} \\
.797 \\
.995 \mathrm{~s} \\
.876 \mathrm{~s} \\
.701 \mathrm{~s}\end{array}$ & $\begin{array}{l}\mathrm{Fe} \\
\mathrm{Fe} \\
\mathrm{Fe} \\
\mathrm{Ni} \\
\mathrm{Fe}\end{array}$ & $\begin{array}{l}3 \\
0 \\
2 \\
5 \\
3\end{array}$ & $\begin{array}{l}242 \\
246 \\
242 \\
238 \\
250\end{array}$ & $\begin{array}{l}241 \\
242 \\
243 \\
243 \\
245\end{array}$ & $\begin{array}{l} \pm 1 \\
-3\end{array}$ & $\begin{array}{ll}\text { IV } & b \\
V & \\
\text { I } & \\
\text { IV } & b\end{array}$ \\
\hline $\begin{array}{r}6677.996 \\
6703.569 \\
05.108 \\
15.387 \\
6717.686\end{array}$ & $\begin{array}{l}\mathrm{B} \\
\mathrm{C} \\
\mathrm{C} \\
\mathrm{D} \\
\mathrm{B}\end{array}$ & $\begin{array}{r}31 \\
17 \\
19 \\
4 \\
20\end{array}$ & $\begin{array}{l}8.235 \mathrm{~s} \\
.820 \mathrm{~s} \\
.352 \mathrm{~s} \\
.635 \\
.940 \mathrm{~s}\end{array}$ & $\begin{array}{c}\mathrm{Fe} \\
\mathrm{Fe} \\
\mathrm{Fe} \\
\mathrm{Cr} ? \mathrm{Fe} ? \\
\mathrm{Ca}\end{array}$ & $\begin{array}{l}5 \\
1 \\
1 \\
1 \\
5\end{array}$ & $\begin{array}{l}239 \\
251 \\
244 \\
248 \\
254\end{array}$ & $\begin{array}{l}246 \\
248 \\
248 \\
248 \\
249\end{array}$ & +9 & III b4 \\
\hline
\end{tabular}


TABLE 1.-A. O. B. S. solar wave lengths-Continued

\begin{tabular}{|c|c|c|c|c|c|c|c|c|c|}
\hline \multirow{2}{*}{ A. O.B.S. $\lambda$} & \multirow{2}{*}{$\begin{array}{l}\text { Proba- } \\
\text { ble } \\
\text { error }\end{array}$} & \multirow{2}{*}{$\begin{array}{l}\text { No. } \\
\text { obs. }\end{array}$} & \multicolumn{3}{|c|}{ Rowland } & \multicolumn{2}{|c|}{$\begin{array}{l}\text { Correction to } \\
\text { Rowland }\end{array}$} & \multirow{2}{*}{ Sun-arc } & \multirow{2}{*}{$\begin{array}{c}\text { Tempera- } \\
\text { ture and } \\
\text { pressure } \\
\text { classes }\end{array}$} \\
\hline & & & $\lambda$ & Iden. & Int. & Obs. & Mean & & \\
\hline $\begin{array}{r}6721.849 \\
26.667 \\
33.157 \\
50.161 \\
52.708\end{array}$ & $\begin{array}{l}\mathrm{C} \\
\mathrm{C} \\
\mathrm{C} \\
\mathrm{B} \\
\mathrm{C}\end{array}$ & $\begin{array}{r}10 \\
14 \\
9 \\
21 \\
11\end{array}$ & $\begin{array}{l}2.096 \mathrm{~s} \\
.925 \mathrm{~s} \\
.410 \\
.407 \mathrm{~s} \\
.965 \mathrm{~s}\end{array}$ & $\begin{array}{l}\mathrm{Fe} \\
\mathrm{Fe} \\
\mathrm{Fe} \\
\mathrm{Fe}\end{array}$ & $\begin{array}{l}2 \\
2 \\
1 \\
3 \\
1\end{array}$ & $\begin{array}{l}247 \\
258 \\
253 \\
246 \\
257\end{array}$ & $\begin{array}{l}249 \\
249 \\
249 \\
250 \\
250\end{array}$ & +10 & IV b \\
\hline $\begin{array}{r}67.781 \\
6772.325 \\
6806.849 \\
10.268 \\
20.375\end{array}$ & $\begin{array}{l}\mathrm{B} \\
\mathrm{C} \\
\mathrm{C} \\
\mathrm{B} \\
\mathrm{B}\end{array}$ & $\begin{array}{l}23 \\
17 \\
10 \\
21 \\
13\end{array}$ & $\begin{array}{r}8.028 \mathrm{~s} \\
.568 \mathrm{~s} \\
7.103 \mathrm{~s} \\
.519 \mathrm{~s} \\
.630 \mathrm{~s}\end{array}$ & $\begin{array}{l}\mathrm{Ni} \\
\mathrm{Ni} \\
\mathrm{Fe} \\
\mathrm{Fe} \\
\mathrm{Fe}\end{array}$ & $\begin{array}{l}4 \\
2 \\
1 \\
3 \\
2\end{array}$ & $\begin{array}{l}247 \\
243 \\
254 \\
251 \\
255\end{array}$ & $\begin{array}{l}250 \\
251 \\
252 \\
252 \\
252\end{array}$ & +3 & $\begin{array}{l}\text { I } \\
\text { V }\end{array}$ \\
\hline $\begin{array}{r}28.600 \\
6839.826 \\
41.344 \\
42.690 \\
43.658\end{array}$ & $\begin{array}{l}\text { B } \\
\text { C } \\
\text { A } \\
\text { C } \\
\text { B }\end{array}$ & $\begin{array}{r}21 \\
8 \\
23 \\
9 \\
21\end{array}$ & $\begin{array}{l}.850 \mathrm{~s} \\
.086 \\
.598 \mathrm{~s} \\
.945 \\
.913 \mathrm{~s}\end{array}$ & $\begin{array}{l}\mathrm{Fe} \\
\mathrm{Fe} \\
\mathrm{Fe} \\
\mathrm{Fe} \\
\mathrm{Fe}\end{array}$ & $\begin{array}{l}2 \\
1 \\
3 \\
1 \\
3\end{array}$ & $\begin{array}{l}250 \\
250 \\
254 \\
255 \\
255\end{array}$ & $\begin{array}{l}253 \\
253 \\
253 \\
253 \\
253\end{array}$ & 4 & $\begin{array}{l}\text { V } \\
\text { V } \\
\text { V }\end{array}$ \\
\hline $\begin{array}{l}55.169 \\
57.250 \\
58.154 \\
62.496 \\
67.221\end{array}$ & $\begin{array}{l}\text { B } \\
\text { D } \\
\text { A } \\
\text { D } \\
\text { A }\end{array}$ & $\begin{array}{r}24 \\
4 \\
24 \\
4 \\
26\end{array}$ & $\begin{array}{l}.419 \mathrm{~s} \\
.515 \\
.415 \\
.760 \\
.457 \mathrm{~s}\end{array}$ & $\begin{array}{l}\mathrm{Fe} \\
\mathrm{Fe} \\
\mathrm{Fe} \\
\mathrm{Fe} \\
\text { Air }\end{array}$ & $\begin{array}{l}3 \\
0 \\
2 \\
1 \\
6\end{array}$ & $\begin{array}{l}250 \\
265 \\
261 \\
264 \\
236\end{array}$ & $\begin{array}{l}254 \\
254 \\
254 \\
254 \\
254\end{array}$ & +10 & V \\
\hline $\begin{array}{l}67.545 \\
69.936 \\
70.945 \\
71.289 \\
72.247\end{array}$ & $\begin{array}{l}\mathrm{A} \\
\mathrm{C} \\
\mathrm{A} \\
\mathrm{A} \\
\mathrm{A}\end{array}$ & $\begin{array}{r}29 \\
4 \\
17 \\
18 \\
31\end{array}$ & $\begin{array}{l}.800 \mathrm{~s} \\
.116 \\
1.180 \mathrm{~s} \\
.532 \mathrm{~s} \\
.486 \mathrm{~s}\end{array}$ & $\begin{array}{l}\text { Air } \\
\text { Air } \\
\text { Air } \\
\text { Air } \\
\text { Air }\end{array}$ & $\begin{array}{r}5 \\
7 \\
8 \\
10 \\
11\end{array}$ & $\begin{array}{l}255 \\
180 \\
235 \\
243 \\
239\end{array}$ & $\begin{array}{l}254 \\
254 \\
242 \\
242 \\
242\end{array}$ & & \\
\hline $\begin{array}{l}72.843 \\
73.795 \\
74.652 \\
75.592 \\
76.718\end{array}$ & $\begin{array}{l}\text { A } \\
\text { A } \\
\text { A } \\
\text { A } \\
\text { A }\end{array}$ & $\begin{array}{l}31 \\
31 \\
31 \\
31 \\
31\end{array}$ & $\begin{array}{r}3.080 \mathrm{~s} \\
4.037 \mathrm{~s} \\
.899 \mathrm{~s} \\
.830 \mathrm{~s} \\
.958 \mathrm{~s}\end{array}$ & $\begin{array}{l}\text { Air } \\
\text { Air } \\
\text { Air } \\
\text { Air } \\
\text { Air }\end{array}$ & $\begin{array}{l}12 \\
12 \\
13 \\
13 \\
13\end{array}$ & $\begin{array}{l}237 \\
242 \\
247 \\
248 \\
240\end{array}$ & $\begin{array}{l}243 \\
243 \\
243 \\
243 \\
243\end{array}$ & & \\
\hline $\begin{array}{l}77.637 \\
79.044 \\
79.929 \\
83.833 \\
85.756\end{array}$ & $\begin{array}{l}\mathrm{A} \\
\mathrm{A} \\
\mathrm{A} \\
\mathrm{A} \\
\mathrm{A}\end{array}$ & $\begin{array}{l}30 \\
31 \\
34 \\
31 \\
31\end{array}$ & $\begin{array}{l}.882 \mathrm{~s} \\
.288 \mathrm{~s} \\
0.172 \mathrm{~s} \\
4.076 \mathrm{~s} \\
6.000 \mathrm{~s}\end{array}$ & $\begin{array}{l}\text { Air } \\
\text { Air } \\
\text { Air } \\
\text { Air } \\
\text { Air }\end{array}$ & $\begin{array}{r}12 \\
12 \\
6 \\
10 \\
11\end{array}$ & $\begin{array}{l}245 \\
244 \\
243 \\
243 \\
244\end{array}$ & $\begin{array}{l}243 \\
243 \\
243 \\
244 \\
244\end{array}$ & & \\
\hline $\begin{array}{l}86.744 \\
88.948 \\
89.903 \\
92.372 \\
93.310\end{array}$ & $\begin{array}{l}\text { B } \\
\text { A } \\
\text { A } \\
\text { A } \\
\text { A }\end{array}$ & $\begin{array}{l}31 \\
31 \\
31 \\
31 \\
31\end{array}$ & $\begin{array}{r}.990 \mathrm{~s} \\
9.192 \mathrm{~s} \\
0.151 \mathrm{~s} \\
.618 \mathrm{~s} \\
.560 \mathrm{~s}\end{array}$ & $\begin{array}{l}\text { Air } \\
\text { Air } \\
\text { Air } \\
\text { Air } \\
\text { Air }\end{array}$ & $\begin{array}{l}12 \\
13 \\
14 \\
14 \\
15\end{array}$ & $\begin{array}{l}246 \\
244 \\
248 \\
246 \\
250\end{array}$ & $\begin{array}{l}244 \\
245 \\
245 \\
245 \\
245\end{array}$ & & \\
\hline $\begin{array}{r}96.040 \\
96.965 \\
6899.955 \\
6900.869 \\
04.118\end{array}$ & $\begin{array}{l}\mathbf{A} \\
\mathbf{A} \\
\mathbf{A} \\
\mathbf{A} \\
\mathbf{A}\end{array}$ & $\begin{array}{l}30 \\
31 \\
31 \\
31 \\
31\end{array}$ & $\begin{array}{r}.289 \mathrm{~s} \\
7.208 \mathrm{~s} \\
0.199 \mathrm{~s} \\
1.117 \mathrm{~s} \\
.362 \mathrm{~s}\end{array}$ & $\begin{array}{l}\text { Air } \\
\text { Air } \\
\text { Air } \\
\text { Air } \\
\text { Air }\end{array}$ & $\begin{array}{l}14 \\
15 \\
14 \\
15 \\
14\end{array}$ & $\begin{array}{l}249 \\
243 \\
244 \\
248 \\
244\end{array}$ & $\begin{array}{l}246 \\
246 \\
246 \\
246 \\
246\end{array}$ & & \\
\hline $\begin{array}{l}05.025 \\
08.535 \\
09.432 \\
13.201 \\
14.092\end{array}$ & $\begin{array}{l}\mathrm{A} \\
\mathrm{A} \\
\mathrm{A} \\
\mathrm{A} \\
\mathrm{A}\end{array}$ & $\begin{array}{l}31 \\
30 \\
31 \\
33 \\
32\end{array}$ & $\begin{array}{l}.271 \mathrm{~s} \\
.783 \mathrm{~s} \\
.676 \mathrm{~s} \\
.448 \mathrm{~s} \\
.337 \mathrm{~s}\end{array}$ & $\begin{array}{l}\text { Air } \\
\text { Air } \\
\text { Air } \\
\text { Air } \\
\text { Air }\end{array}$ & $\begin{array}{l}14 \\
13 \\
13 \\
11 \\
11\end{array}$ & $\begin{array}{l}246 \\
248 \\
244 \\
247 \\
245\end{array}$ & $\begin{array}{l}246 \\
247 \\
247 \\
247 \\
247\end{array}$ & & \\
\hline $\begin{array}{l}\text { 14. } 573 \\
16.686 \\
18.121 \\
19.002 \\
23.302\end{array}$ & $\begin{array}{l}\text { B } \\
\text { B } \\
\text { A } \\
\text { A } \\
\text { A }\end{array}$ & $\begin{array}{l}20 \\
21 \\
33 \\
33 \\
31\end{array}$ & $\begin{array}{r}.823 \mathrm{~s} \\
.948 \mathrm{~s} \\
.370 \mathrm{~s} \\
9.250 \mathrm{~s} \\
.553 \mathrm{~s}\end{array}$ & $\begin{array}{l}\mathrm{Ni} \\
\text { Fe } \\
\text { Air } \\
\text { Air } \\
\text { Air }\end{array}$ & $\begin{array}{l}4 \\
2 \\
9 \\
9 \\
9\end{array}$ & $\begin{array}{l}250 \\
262 \\
249 \\
248 \\
251\end{array}$ & $\begin{array}{l}247 \\
248 \\
248 \\
248 \\
249\end{array}$ & & \\
\hline $\begin{array}{l}24.173 \\
28.729 \\
29.595 \\
33.615 \\
33.804\end{array}$ & $\begin{array}{l}\text { A } \\
\text { A } \\
\text { B } \\
\text { C } \\
\text { D }\end{array}$ & $\begin{array}{r}31 \\
21 \\
13 \\
5 \\
7\end{array}$ & $\begin{array}{l}.427 \mathrm{~s} \\
.977 \mathrm{~s} \\
.840 \mathrm{~s} \\
.887 \\
4.075\end{array}$ & $\begin{array}{l}\text { Air } \\
\text { Air } \\
\text { Air } \\
\text { Fe } \\
\text { Air }\end{array}$ & $\begin{array}{l}9 \\
4 \\
4 \\
2 \\
2\end{array}$ & $\begin{array}{l}254 \\
248 \\
245 \\
272 \\
271\end{array}$ & $\begin{array}{l}249 \\
249 \\
249 \\
250 \\
250\end{array}$ & & \\
\hline $\begin{array}{r}34.422 \\
35.280 \\
37.703 \\
39.618 \\
6940.190\end{array}$ & $\begin{array}{l}\mathrm{B} \\
\mathrm{B} \\
\mathrm{B} \\
\mathrm{B} \\
\mathrm{B}\end{array}$ & $\begin{array}{r}13 \\
14 \\
19 \\
13 \\
6\end{array}$ & $\begin{array}{l}.670 \mathrm{~s} \\
.530 \mathrm{~s} \\
.957 \\
.875 \\
.436\end{array}$ & $\begin{array}{l}\text { Air } \\
\text { Air } \\
\text { Air } \\
\text { Air } \\
\text { Air }\end{array}$ & $\begin{array}{l}2 \\
2 \\
2 \\
2 \\
2\end{array}$ & $\begin{array}{l}248 \\
250 \\
254 \\
257 \\
246\end{array}$ & $\begin{array}{l}250 \\
250 \\
251 \\
251 \\
251\end{array}$ & & \\
\hline
\end{tabular}


TABLE 1.-A. O. B. S. solar wave lengths-Continued

\begin{tabular}{|c|c|c|c|c|c|c|c|c|c|}
\hline \multirow{2}{*}{ A.O.B.S. $\lambda$} & \multirow{2}{*}{$\begin{array}{c}\text { Proba- } \\
\text { ble } \\
\text { error }\end{array}$} & \multirow{2}{*}{$\begin{array}{l}\text { No. } \\
\text { obs. }\end{array}$} & \multicolumn{3}{|c|}{ Rowland } & \multicolumn{2}{|c|}{$\begin{array}{l}\text { Correction to } \\
\text { Rowland }\end{array}$} & \multirow{2}{*}{ Sun-are } & \multirow{2}{*}{$\begin{array}{c}\text { Tempera } \\
\text { ture and } \\
\text { pressure } \\
\text { classes }\end{array}$} \\
\hline & & & $\lambda$ & Iden. & Int. & Obs. & Mean & & \\
\hline $\begin{array}{r}6942.152 \\
43.802 \\
45.214 \\
47.534 \\
51.248\end{array}$ & $\begin{array}{l}\mathbf{A} \\
\mathbf{B} \\
\mathbf{B} \\
\mathbf{B} \\
\mathbf{B}\end{array}$ & $\begin{array}{r}5 \\
15 \\
17 \\
14 \\
13\end{array}$ & $\begin{array}{l}.402 \\
4.060 \\
.477 \\
.782 \mathrm{~s} \\
.518\end{array}$ & $\begin{array}{l}\text { Air } \\
\text { Air } \\
\text { Fe } \\
\text { Air-Fe } \\
\mathrm{Fe}\end{array}$ & $\begin{array}{l}2 \\
3 \\
5 \\
5 \\
1\end{array}$ & $\begin{array}{l}250 \\
258 \\
263 \\
248 \\
270\end{array}$ & $\begin{array}{l}251 \\
251 \\
252 \\
252 \\
252\end{array}$ & +10 & IV \\
\hline $\begin{array}{l}56.426 \\
59.452 \\
61.260 \\
78.403 \\
78.862\end{array}$ & $\begin{array}{l}\text { C } \\
\text { A } \\
\text { A } \\
\text { C } \\
\text { B }\end{array}$ & $\begin{array}{r}7 \\
16 \\
16 \\
3 \\
13\end{array}$ & $\begin{array}{r}.660 \mathrm{~s} \\
.704 \mathrm{~s} \\
.515 \mathrm{~s} \\
.670 \mathrm{~s} \\
9.120\end{array}$ & $\begin{array}{l}\text { Air } \\
\text { Air } \\
\text { Air } \\
\text { Cr } \\
\text { Fe }\end{array}$ & $\begin{array}{l}4 \\
3 \\
4 \\
1 \\
2\end{array}$ & $\begin{array}{l}234 \\
252 \\
255 \\
267 \\
258\end{array}$ & $\begin{array}{l}253 \\
253 \\
254 \\
257 \\
257\end{array}$ & +4 & IV \\
\hline $\begin{array}{l}86.580 \\
88.518 \\
88.986 \\
90.373 \\
93.517\end{array}$ & $\begin{array}{l}\mathrm{C} \\
\mathrm{D} \\
\mathrm{B} \\
\mathrm{C} \\
\mathrm{C}\end{array}$ & $\begin{array}{r}14 \\
4 \\
14 \\
7 \\
10\end{array}$ & $\begin{array}{l}.833 \mathrm{~s} \\
.805 \\
9.237 \mathrm{~s} \\
.632 \\
.776\end{array}$ & $\begin{array}{l}\text { Air } \\
\text { Fe } \\
\text { Air } \\
\text { Air } \\
\text { Air }\end{array}$ & $\begin{array}{l}3 \\
0 \\
3 \\
1 \\
2\end{array}$ & $\begin{array}{l}253 \\
287 \\
251 \\
259 \\
259\end{array}$ & $\begin{array}{l}259 \\
259 \\
259 \\
259 \\
260\end{array}$ & & \\
\hline $\begin{array}{r}94.107 \\
98.963 \\
6999.882 \\
7003.574 \\
04.747\end{array}$ & $\begin{array}{l}\mathrm{D} \\
\mathrm{C} \\
\mathrm{C} \\
\mathrm{C} \\
\mathrm{C}\end{array}$ & $\begin{array}{r}7 \\
12 \\
9 \\
4 \\
12\end{array}$ & $\begin{array}{l}.360 \\
9.223 \\
0.155 \mathrm{~s} \\
.837 \\
.995\end{array}$ & $\begin{array}{l}\text { Air } \\
\text { Air } \\
\text { Fe } \\
\text { Si? } \\
\text { Air }\end{array}$ & $\begin{array}{l}2 \\
2 \\
1 \\
1 \\
2\end{array}$ & $\begin{array}{l}253 \\
260 \\
273 \\
263 \\
248\end{array}$ & $\begin{array}{l}260 \\
261 \\
261 \\
262 \\
262\end{array}$ & 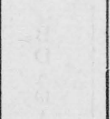 & \\
\hline $\begin{array}{l}11.330 \\
22.956 \\
23.504 \\
27.479 \\
34.909\end{array}$ & $\begin{array}{l}\mathrm{D} \\
\mathrm{B} \\
\mathrm{C} \\
\mathrm{B} \\
\mathrm{C}\end{array}$ & $\begin{array}{r}11 \\
8 \\
10 \\
10 \\
8\end{array}$ & $\begin{array}{r}.590 \mathrm{~s} \\
3.230 \mathrm{~s} \\
.770 \mathrm{~s} \\
.740 \mathrm{~s} \\
5.170 \mathrm{~s}\end{array}$ & $\begin{array}{l}\text { Air } \\
\text { Fe } \\
\text { Air } \\
\text { Air }\end{array}$ & $\begin{array}{l}2 \\
2 \\
2 \\
2 \\
2\end{array}$ & $\begin{array}{l}260 \\
274 \\
266 \\
261 \\
261\end{array}$ & $\begin{array}{l}263 \\
265 \\
266 \\
266 \\
267\end{array}$ & & \\
\hline $\begin{array}{r}38.219 \\
39.796 \\
50.858 \\
56.999 \\
7084.981\end{array}$ & $\begin{array}{l}\text { C } \\
B \\
D \\
\text { C } \\
C\end{array}$ & $\begin{array}{r}9 \\
11 \\
6 \\
4 \\
4\end{array}$ & $\begin{array}{l}.500 \mathrm{~s} \\
.053 \mathrm{~s} \\
1.115 \\
7.260 \\
5.265\end{array}$ & $\begin{array}{l}\text { Fe } \\
\text { Air } \\
\text { Air } \\
\text { Air } \\
\text { Co }\end{array}$ & $\begin{array}{l}1 \\
1 \\
0 \\
0 \\
2\end{array}$ & $\begin{array}{l}281 \\
267 \\
257 \\
261 \\
284\end{array}$ & $\begin{array}{l}267 \\
268 \\
269 \\
269 \\
271\end{array}$ & & \\
\hline $\begin{array}{r}7122.206 \\
30.928 \\
32.988 \\
7148.159\end{array}$ & $\begin{array}{l}D \\
D \\
D \\
D\end{array}$ & $\begin{array}{l}5 \\
5 \\
5 \\
5\end{array}$ & $\begin{array}{r}.484 \\
1.204 \\
3.263 \\
.435\end{array}$ & $\begin{array}{l}\mathrm{Ni} \\
\mathrm{Fe} \\
\mathrm{Fe} \\
\mathrm{Ca}\end{array}$ & $\begin{array}{l}4 \\
3 \\
1 \\
3\end{array}$ & $\begin{array}{l}278 \\
276 \\
275 \\
276\end{array}$ & $\begin{array}{l}275 \\
276 \\
276 \\
277\end{array}$ & $\begin{array}{l} \pm 0 \\
+14\end{array}$ & $\begin{array}{l}\text { IV } \\
\text { II }\end{array}$ \\
\hline
\end{tabular}

TABLE 2.-Corrections to Rowland's wave lengths (3590-7142)

\begin{tabular}{|c|c|c|c|c|c|}
\hline Wave length & Correction & Wave length & Correction & Wave length & Correction \\
\hline $\begin{array}{l}3590-3650 \\
3651-3940 \\
3941-3952 \\
3953-3960 \\
3961-3970\end{array}$ & $\begin{array}{c}-0.141 \mathrm{~A} \\
.140 \\
.141 \\
.142 \\
.143\end{array}$ & $\begin{array}{l}4165-4176 \\
4177-4187 \\
4188-4199 \\
4200-4213 \\
4214-4244\end{array}$ & $\begin{array}{l}.163 \\
.162 \\
.161 \\
.160 \\
.159\end{array}$ & $\begin{array}{l}4567-4587 \\
4588-4597 \\
4598-4634 \\
4635-4648 \\
4649-4671\end{array}$ & $\begin{array}{l}.172 \\
.173 \\
.174 \\
.173 \\
.172\end{array}$ \\
\hline $\begin{array}{l}3971-3975 \\
3976-3978 \\
3979-3985 \\
3986-3992 \\
3993-3997\end{array}$ & $\begin{array}{l}.144 \\
.145 \\
.146 \\
.147 \\
.148\end{array}$ & $\begin{array}{l}4245-4295 \\
4296-4310 \\
4311-4320 \\
4321-4330 \\
4331-4357\end{array}$ & $\begin{array}{l}.158 \\
.159 \\
.160 \\
.161 \\
.162\end{array}$ & $\begin{array}{l}4672-4690 \\
4691-4708 \\
4709-4721 \\
4722-4729 \\
4730-4734\end{array}$ & $\begin{array}{l}.173 \\
.174 \\
.175 \\
.176 \\
.177\end{array}$ \\
\hline $\begin{array}{l}3998-4000 \\
4001-4007 \\
4008-4018 \\
4019-4024 \\
4025-4028\end{array}$ & $\begin{array}{l}.149 \\
.150 \\
.151 \\
.152 \\
.153\end{array}$ & $\begin{array}{l}4358-4373 \\
4374-4378 \\
4379-4397 \\
4398-4414 \\
4415-4426\end{array}$ & $\begin{array}{l}.161 \\
.160 \\
.159 \\
.160 \\
.161\end{array}$ & $\begin{array}{l}4735-4738 \\
4739-4744 \\
4745-4749 \\
4750-4753 \\
4754-4760\end{array}$ & $\begin{array}{l}.178 \\
.179 \\
.180 \\
.181 \\
.182\end{array}$ \\
\hline $\begin{array}{l}4029-4037 \\
4038-4090 \\
4091-4116 \\
4117-4123 \\
4124-4129\end{array}$ & $\begin{array}{l}.154 \\
.155 \\
.156 \\
.155 \\
.154\end{array}$ & $\begin{array}{l}4427-4435 \\
4436-4488 \\
4489-4500 \\
4501-4514 \\
4515-4523\end{array}$ & $\begin{array}{l}.162 \\
.163 \\
.164 \\
.165 \\
.166\end{array}$ & $\begin{array}{l}4761-4773 \\
4774-4785 \\
4786-4815 \\
4816-4825 \\
4826-4835\end{array}$ & $\begin{array}{l}.183 \\
.184 \\
.185 \\
.184 \\
.183\end{array}$ \\
\hline $\begin{array}{l}4130-4135 \\
4136-4141 \\
4142 \\
4143-4152 \\
4153-4164\end{array}$ & $\begin{array}{l}.153 \\
.152 \\
(1) \\
.163 \\
.164\end{array}$ & $\begin{array}{l}4524-4535 \\
4536-4550 \\
4551-4558 \\
4559-4562 \\
4563-4566\end{array}$ & $\begin{array}{l}.167 \\
.168 \\
.169 \\
.170 \\
.171\end{array}$ & $\begin{array}{l}4836-4842 \\
4843-4848 \\
4849-4853 \\
4854-4860\end{array}$ & $\begin{array}{l}.182 \\
.181 \\
.180 \\
.179\end{array}$ \\
\hline
\end{tabular}

1 Discontinuity. 
TABLE 2.-Corrections to Rowland's wave lengths (3590-7142)-Continued

\begin{tabular}{|c|c|c|c|c|c|}
\hline Wave length & Correction & Wave length & Correction & Wave length & Correction \\
\hline $\begin{array}{l}4861-4865 \\
4866-4877 \\
4878-4884 \\
4885-4892 \\
4893-4900\end{array}$ & $\begin{array}{r}-0.178 \\
.177 \\
.176 \\
.175 \\
.174\end{array}$ & $\begin{array}{l}5497-5502 \\
5503-5506 \\
5507-5511 \\
5512-5515 \\
5516-5519\end{array}$ & $\begin{array}{l}.206 \\
.207 \\
.208 \\
.209 \\
.210\end{array}$ & $\begin{array}{l}6513-6516 \\
6517-6519 \\
6520-6522 \\
6523-6524 \\
6525-6528\end{array}$ & $\begin{array}{l}.226 \\
.227 \\
.228 \\
.229 \\
.230\end{array}$ \\
\hline $\begin{array}{l}4901-4907 \\
4908-4920 \\
4921-5050 \\
5051-5067 \\
5068-5080\end{array}$ & $\begin{array}{l}.173 \\
.172 \\
.171 \\
.170 \\
.169\end{array}$ & $\begin{array}{l}5520-5524 \\
5525-5528 \\
5529-5532 \\
5533-5537 \\
5538-5543\end{array}$ & $\begin{array}{l}.211 \\
.212 \\
.213 \\
.214 \\
.215\end{array}$ & $\begin{array}{l}6529-6531 \\
6532-6534 \\
6535-6537 \\
6538-6541 \\
6542-6547\end{array}$ & $\begin{array}{l}.231 \\
.232 \\
.233 \\
.234 \\
.235\end{array}$ \\
\hline $\begin{array}{l}5081-5095 \\
5096-5110 \\
5111-5130 \\
5131-5160 \\
5161-5175\end{array}$ & $\begin{array}{l}.168 \\
.167 \\
.166 \\
.165 \\
.164\end{array}$ & $\begin{array}{l}5544-5565 \\
5566-5604 \\
5605-5650 \\
5651-5660 \\
5661-5681\end{array}$ & $\begin{array}{l}.216 \\
.217 \\
.218 \\
.217 \\
.216\end{array}$ & $\begin{array}{l}6548-6553 \\
6554-6566 \\
6567-6581 \\
6582-6592 \\
6593-6604\end{array}$ & $\begin{array}{l}.236 \\
.237 \\
.238 \\
.239 \\
.240\end{array}$ \\
\hline $\begin{array}{l}5176-5188 \\
5189-5200 \\
5201-5212 \\
5218 \\
5224-5225\end{array}$ & $\begin{array}{l}.163 \\
.162 \\
.161 \\
(1) \\
.155\end{array}$ & $\begin{array}{l}5682-5700 \\
5701-5725 \\
5726-5737 \\
5738-5748 \\
5749-5762\end{array}$ & $\begin{array}{l}.215 \\
.214 \\
.213 \\
.212 . \\
.211\end{array}$ & $\begin{array}{l}6605-6617 \\
6618-6631 \\
6632-6643 \\
6644-6656 \\
6657-6669\end{array}$ & $\begin{array}{l}.241 \\
.242 \\
.243 \\
.244 \\
.245\end{array}$ \\
\hline $\begin{array}{l}5226-5230 \\
5231-5234 \\
5235-5238 \\
5239-5242 \\
5243-5246\end{array}$ & $\begin{array}{l}.156 \\
.157 \\
.158 \\
.159 \\
.160\end{array}$ & $\begin{array}{l}5763-5772 \\
5773-5795 \\
5805-5819 \\
5820-5870 \\
5871-5882\end{array}$ & $\begin{array}{l}.210 \\
.209 \\
.214 \\
.213 \\
.214\end{array}$ & $\begin{array}{l}6670-6681 \\
6682-6695 \\
6696-6718 \\
6719-6738 \\
6739-6767\end{array}$ & $\begin{array}{l}.246 \\
.247 \\
.248 \\
.249 \\
.250\end{array}$ \\
\hline $\begin{array}{l}5247-5250 \\
5251-5254 \\
5255-5258 \\
5259-5262 \\
5263-5266\end{array}$ & $\begin{array}{l}.161 \\
.162 \\
.163 \\
.164 \\
.165\end{array}$ & $\begin{array}{l}5883-5894 \\
5895-5937 \\
5938-5972 \\
5973-5991 \\
5992-6007\end{array}$ & $\begin{array}{l}.215 \\
.216 \\
.217 \\
.218 \\
.217\end{array}$ & $\begin{array}{l}6768-6794 \\
6795-6822 \\
6823-6847 \\
6848-6869 \\
6870\end{array}$ & $\begin{array}{l}.251 \\
.252 \\
.253 \\
.254 \\
(1)\end{array}$ \\
\hline $\begin{array}{l}5267-5271 \\
5272-5275 \\
5276-5280 \\
5281-5286 \\
5287-5291\end{array}$ & $\begin{array}{l}.166 \\
.167 \\
.168 \\
.169 \\
.170\end{array}$ & $\begin{array}{l}6008-6024 \\
6025-6044 \\
6045-6066 \\
6067-6084 \\
6085-6104\end{array}$ & $\begin{array}{l}.216 \\
.215 \\
.214 \\
.213 \\
.212\end{array}$ & $\begin{array}{l}6870-6872 \\
6873-6879 \\
6880-6886 \\
6887-6895 \\
6896-6907\end{array}$ & $\begin{array}{l}.242 \\
.243 \\
.244 \\
.245 \\
.246\end{array}$ \\
\hline $\begin{array}{l}5292-5297 \\
5298-5302 \\
5303-5307 \\
5308-5311 \\
5312-5316\end{array}$ & $\begin{array}{l}.171 \\
.172 \\
.173 \\
.174 \\
.175\end{array}$ & $\begin{array}{l}6105-6123 \\
6124-6162 \\
6163-6176 \\
6177-6189 \\
6190-6196\end{array}$ & $\begin{array}{l}.211 \\
.210 \\
.211 \\
.212 \\
.211\end{array}$ & $\begin{array}{l}6908-6913 \\
6914-6920 \\
6921-6929 \\
6930-6937 \\
6938-6943\end{array}$ & $\begin{array}{l}.247 \\
.248 \\
.249 \\
.250 \\
.251\end{array}$ \\
\hline $\begin{array}{l}5317-5320 \\
5321-5324 \\
5325-5329 \\
5330-5333 \\
5334-5338\end{array}$ & $\begin{array}{l}.176 \\
.177 \\
.178 \\
.179 \\
.180\end{array}$ & $\begin{array}{l}6197-6203 \\
6204-6240 \\
6241-6263 \\
6264-6272 \\
6273-6292\end{array}$ & $\begin{array}{l}.210 \\
.209 \\
.210 \\
.209 \\
.208\end{array}$ & $\begin{array}{l}6944-6952 \\
6953-6959 \\
6960-6964 \\
6965-6969 \\
6970-6974\end{array}$ & $\begin{array}{l}.252 \\
.253 \\
.254 \\
.255 \\
.256\end{array}$ \\
\hline $\begin{array}{l}5339-5342 \\
5343-5346 \\
5347-5350 \\
5351-5354 \\
5355-5358\end{array}$ & $\begin{array}{l}.181 \\
.182 \\
.183 \\
.184 \\
.185\end{array}$ & $\begin{array}{l}6293-6297 \\
6298-6300 \\
6301-6303 \\
6304-6307 \\
6308-6315\end{array}$ & $\begin{array}{l}.209 \\
.210 \\
.211 \\
.212 \\
.213\end{array}$ & $\begin{array}{l}6975-6980 \\
6981-6985 \\
6986-6990 \\
6991-6996 \\
6997-7001\end{array}$ & $\begin{array}{l}.257 \\
.258 \\
.259 \\
.260 \\
.261\end{array}$ \\
\hline $\begin{array}{l}5359-5361 \\
5362-5365 \\
5366-5369 \\
5370-5373 \\
5374-5376\end{array}$ & $\begin{array}{l}.186 \\
.187 \\
.188 \\
.189 \\
.190\end{array}$ & $\begin{array}{l}6316-6327 \\
6328-6355 \\
6356-6361 \\
6362-6368 \\
6369-6375\end{array}$ & $\begin{array}{l}.214 \\
.215 \\
.214 \\
.213 \\
.212\end{array}$ & $\begin{array}{l}7002-7006 \\
7007-7010 \\
7011-7016 \\
7017-7022 \\
7023-7028\end{array}$ & $\begin{array}{l}.262 \\
.263 \\
.264 \\
.265 \\
.266\end{array}$ \\
\hline $\begin{array}{l}5377-5379 \\
5380-5383 \\
5384-5386 \\
5387-5389 \\
5390-5393\end{array}$ & $\begin{array}{l}.191 \\
.192 \\
.193 \\
.194 \\
.195\end{array}$ & $\begin{array}{l}6376-6444 \\
6445-6453 \\
6454-6459 \\
6460-6463 \\
6464-6467\end{array}$ & $\begin{array}{l}.211 \\
.212 \\
.213 \\
.214 \\
.215\end{array}$ & $\begin{array}{l}7029-7036 \\
7037-7049 \\
7050-7061 \\
7062-7073 \\
7074-7085\end{array}$ & $\begin{array}{l}.267 \\
.268 \\
.269 \\
.270 \\
.271\end{array}$ \\
\hline $\begin{array}{l}5394-5396 \\
5397-5399 \\
5400-5402 \\
5403-5405 \\
5406-5408\end{array}$ & $\begin{array}{l}.196 \\
.197 \\
.198 \\
.199 \\
.200\end{array}$ & $\begin{array}{l}6468-6473 \\
6474-6477 \\
6478-6482 \\
6483-6486 \\
6487-6490\end{array}$ & $\begin{array}{l}.216 \\
.217 \\
.218 \\
.219 \\
.220\end{array}$ & $\begin{array}{l}7086-7096 \\
7097-7107 \\
7108-7118 \\
7119-7129 \\
7130-7141\end{array}$ & $\begin{array}{l}.272 \\
.273 \\
.274 \\
.275 \\
.276\end{array}$ \\
\hline $\begin{array}{l}5409-5412 \\
5413-5422 \\
5423-5438 \\
5439-5487 \\
5488-5496\end{array}$ & $\begin{array}{l}.201 \\
.202 \\
.203 \\
.204 \\
.205\end{array}$ & $\begin{array}{l}6491-6495 \\
6496-6499 \\
6500-6504 \\
6505-6508 \\
6509-6512\end{array}$ & $\begin{array}{l}.221 \\
.222 \\
.223 \\
.224 \\
.225\end{array}$ & 7142 & .277 \\
\hline
\end{tabular}

1 Discontinuity.

Washington, May 23, 1928. 


$$
\text { . }
$$

